\title{
Quiet Sun Magnetic Fields
}

\author{
Dissertation \\ zur Erlangung des Doktorgrades \\ der Mathematisch-Naturwissenschaftlichen Fakultäten \\ der Georg-August-Universität zu Göttingen
}

\author{
vorgelegt von \\ Itahiza Francisco Domínguez Cerdeña \\ aus Fuerteventura \\ (Kanarische Inseln, Spanien)
}


D7

Referent:

Korreferent:

Tag der mündlichen Prüfung:
Prof. Dr. F. Kneer

Prof. Dr. S. Dreizler

14.7.2004 


\section{To my OWn Sun, Nuribel AND TO MY PARENTS}





\section{Contents}

$\begin{array}{ll}\text { Abstract } & 1\end{array}$

1 Introduction $\quad 3$

1.1 The Sun .......................... 3

1.2 Quiet Sun magnetism. . . . . . . . . . . . . . 4

2 Polarimetry 9

2.1 Zeeman effect . . . . . . . . . . . . . . . . 10

2.2 Radiative Transfer . . . . . . . . . . . . . . . . . . . . . 12

2.3 The controversy on visible and infrared lines . . . . . . . . . . . . 14

3 High spatial resolution polarimetry 17

3.1 Speckle reconstruction . . . . . . . . . . . . . . . . . . . 17

3.2 Observations: FPI . . . . . . . . . . . . . . 20

3.3 Data Analysis . . . . . . . . . . . . . . . . . 23

3.3.1 Narrow-band reconstruction . . . . . . . . . . 23

3.3.2 Crosstalk .................. 25

3.3.3 Magnetograms . . . . . . . . . . . . . 26

3.4 Results . . . . . . . . . . . . . . . . . . . . 29

3.4.1 Flux density . . . . . . . . . . . . . . . . . 29

3.4.2 Magnetic field strength . . . . . . . . . . . . 31

3.4.3 Spatial distribution . . . . . . . . . . . . . . 36

3.4.4 Temporal evolution . . . . . . . . . . . . . . 38

3.4.5 Mesogranulation ................. 41

3.4.6 Consistency . . . . . . . . . . . . . . . . . 42 
3.4.7 The effect of the seeing . . . . . . . . . . . . . 43

4 Simultaneous visible and IR observations 47

4.1 Observations . . . . . . . . . . . . . . . . . . . 47

4.2 Data reduction . . . . . . . . . . . . . . . . . 49

4.2 .1 Wavelength calibration . . . . . . . . . . . . . . 51

4.2 .2 Alignment and spatial resolution . . . . . . . . . . . 53

4.3 Magnetograms . . . . . . . . . . . . . . . . . . 56

4.4 PCA classification . . . . . . . . . . . . . . . . . . . . 59

4.5 Milne-Eddington inversion . . . . . . . . . . . . . . . 60

4.5.1 Magnetic field strength . . . . . . . . . . . . . . . . . 65

4.5 .2 Velocities . . . . . . . . . . . . . . . 71

4.5.3 Probability Density Function (PDF) _ . . . . . . . 73

4.6 MISMA inversion . . . . . . . . . . . . . . . . . 75

4.6 .1 Independent inversion . . . . . . . . . . . . . . 78

4.6.2 Magnetic field strength . . . . . . . . . . . . . . . . 82

4.6.3 Magnetic flux and filling factor . . . . . . . . . . . 87

4.6.4 Probability Density Function (PDF) _ . . . . . . . . 90

4.6.5 Simultaneous inversions _. . . . . . . . . . . . . . 93

$\begin{array}{lll}5 & \text { Summary and conclusions } & 97\end{array}$

$\begin{array}{ll}\text { References } & 101\end{array}$

$\begin{array}{ll}\text { Acknowledgements } & 109\end{array}$

$\begin{array}{ll}\text { Lebenslauf } & 111\end{array}$ 


\section{Abstract}

The aim of this Thesis is the study of the quiet Sun magnetic fields. They are characterized by weak polarization signals due to their small-scale coverage of the solar surface, but with the availability of new, high-sensitivity instruments this subject has obtained increasing interest during the last years. This work approaches the topic in two ways taking advantage of two brand-new observational techniques:

- The spatial resolution is improved compared with previous observations of quiet Sun by the use of the Göttingen Fabry-Perot Interferometer (FPI) at the Vacuum Tower Telescope (VTT, at the Observatorio del Teide, Tenerife). Speckle reconstruction techniques applied to the observations produce two-dimensional spectra of intensity and circular polarization (Stokes $I$ and Stokes $V$ ) with a spatial resolution of 0.5 and a fair polarimetric sensitivity $\left(5 \cdot 10^{-3} I_{c}\right)$. The magnetograms computed for the Fe I lines at 6301.5 and $6302.5 \AA$ exhibit magnetic patches covering $45 \%$ of the observed area with an unsigned magnetic flux density of $20 \mathrm{G}$, which exceeds all previous measurements. The ratio method applied to the magnetic fluxes inferred from the two lines yields the detection of $\mathrm{kG}$ magnetic fields.

- The second part of the work presents the first simultaneous spectropolarimetric observations in the infrared and the visible ranges using the highly sensitive instruments operated in two different telescopes (VTT and THEMIS at the Observatorio del Teide). The two-dimensional observation, by means of scanning part of the solar image, was carried out in the Fe I lines at $6301.5,6302.5,15648.5$ and $15652.8 \AA$. A co-alignment, or common pointing of the two telescopes of $1^{\prime \prime}$ was achieved, which corresponds to a time lag between the observations of only one minute. The seeing was fairly good, 1".5. The study of Stokes $V$ using inversion techniques, under the Milne-Eddington approximation, leads to the finding of the coexistence of $\mathrm{kG}$ magnetic fields (traced by the visible lines) and sub-kG fields (traced by the IR lines). Finally, inversions under the MISMA hypothesis (MIcro-Structured Magnetic Atmosphere) assuming a two-magneticcomponent model, were applied to the IR and visible lines. The resulting models inferred from the IR lines present a large number of analyzed pixels (50-65\%) with $\mathrm{kG}$ field strengths co-existing with sub-kG fields. These results provide an estimation of the probability density function (PDF) of finding a specific intrinsic field in the region. The contribution of the $\mathrm{kG}$ fields contains most of the unsigned magnetic flux although they occupy a smaller fraction of the field of view than the sub-kG fields. 


\section{Chapter 1}

\section{Introduction}

\subsection{The Sun}

The Sun can be understood as a big, close to spherically symmetric conglomerate of ionized gas (which is called plasma), self gravitating and radiating, with a diameter of 700000 kilometers. Further, the Sun is a huge energy source, not only in form of electromagnetic radiation but also in form of mass ejection, in an explosive way in the case of flares, or in a continuous and relatively quiet way in the case of the solar wind. It also emits energy in form of neutrinos, the waste of the nuclear reactions occurring in the solar core. This nuclear fusion is the main generator of the solar energy, producing highly energetic photons which spend a million years on their travel through the Sun to finally arrive at the solar surface, from where they are radiated as visible light.

Moreover the Sun rotates, not in a uniform way but faster inside than outside. The Sun vibrates, and the measurements of the modes of these vibrations allow us to "penetrate" into the Sun's interior. Such measurements are useful to test theoretical models of the Sun's structure. The standard model predicts in the core a temperature of fifteen million Kelvin and a density of one hundred and fifty grams per cubic centimeter. The models infer that the energy transfer is radiative in the inner Sun until a layer where this form of energy transport is not efficient enough and the Sun is "forced" to use convection to bring all this energy up to the photosphere where it is radiated into the space reaching Earth, heating our atmosphere and permitting the existence of life.

How could one define the surface of the Sun? To conceive the outer boundary of the Sun is difficult, because as one moves from the solar center outward, the density decreases steadily, but even the Earth is embedded in the solar wind. The end of the Sun can be considered as the magnetopause, where the solar wind collides with the interstellar medium, thousands of millions of kilometers away. 
However, it is usual to define the solar surface as the place where most of the photons escape freely and can reach us, that is at the optical depth $\tau=1$.

Magnetic fields make the Sun an interesting star from differennt points of view. Their dynamics and distributions generate a wealth of phenomena with implications that reach Earth and us. The magnetic field is responsible for a fauna of structures on the surface, like sunspots, faculae, bright points, filaments, plages or spiculae. Reconnexion of magnetic flux tubes causes flares, violent explosions in which nuclear reactions may happen. Magnetic fields are the ingredients to heat the enigmatic corona to a million Kelvin and more. They are also closely related with the eleven years solar cycle, which influences the climate on Earth. They cause the solar storms, which may destroy space devices and generate the aurorae borealis.

From the stellar point of view, the Sun is just a normal star from the middlelower part of the main sequence, of spectral type G2 and thus belonging to the very abundant group of late-type stars. What makes the Sun a special stellar object is its small distance to us. The Sun is, with the exception of indirect measurements of other stars, still the only star which one can resolve spatially. The best resolution obtained with today's telescopes of $0{ }^{\prime} 11$ corresponds to 70 kilometers on the solar surface, allowing detailed studies not feasible with other stars

The Sun can be considered as a laboratory in stellar astrophysics, plasma physics, atomic physics, fluid mechanics, which can be used to study fundamental physics processes.

\subsection{Quiet Sun magnetism.}

In intensity images of the Sun one can see large structures produced by the solar magnetism in the photosphere, like sunspots or plages. To detect more features of the magnetism it is necessary to use special filters or magnetograms. Figure 1.1 shows a full disk magnetogram of the Sun, where bright and dark areas represent opposite polarities of the magnetic flux. Strongest signals, corresponding to sunspots and plages, are distributed at both sides of the solar equator in the activity belts. In addition, the solar surface is covered by many magnetic patches forming a sort of net. Usually these patches appear stronger near active regions than in the quiet Sun. The net is known as the solar network (see enlargement in Fig. 1.1), and it is the concentration of magnetic flux in the boundaries of the supergranular cells (see Livingston \& Harvey 1975; Smithson 1975). Supergranulation is a convection pattern visible in form of horizontal motions of plasma with scales of about $20 \mathrm{Mm}(1 \mathrm{Mm}=1$ Megameter=1000 km).

In such magnetogram (Fig. 1.1) most of the solar surface seems to be non- 


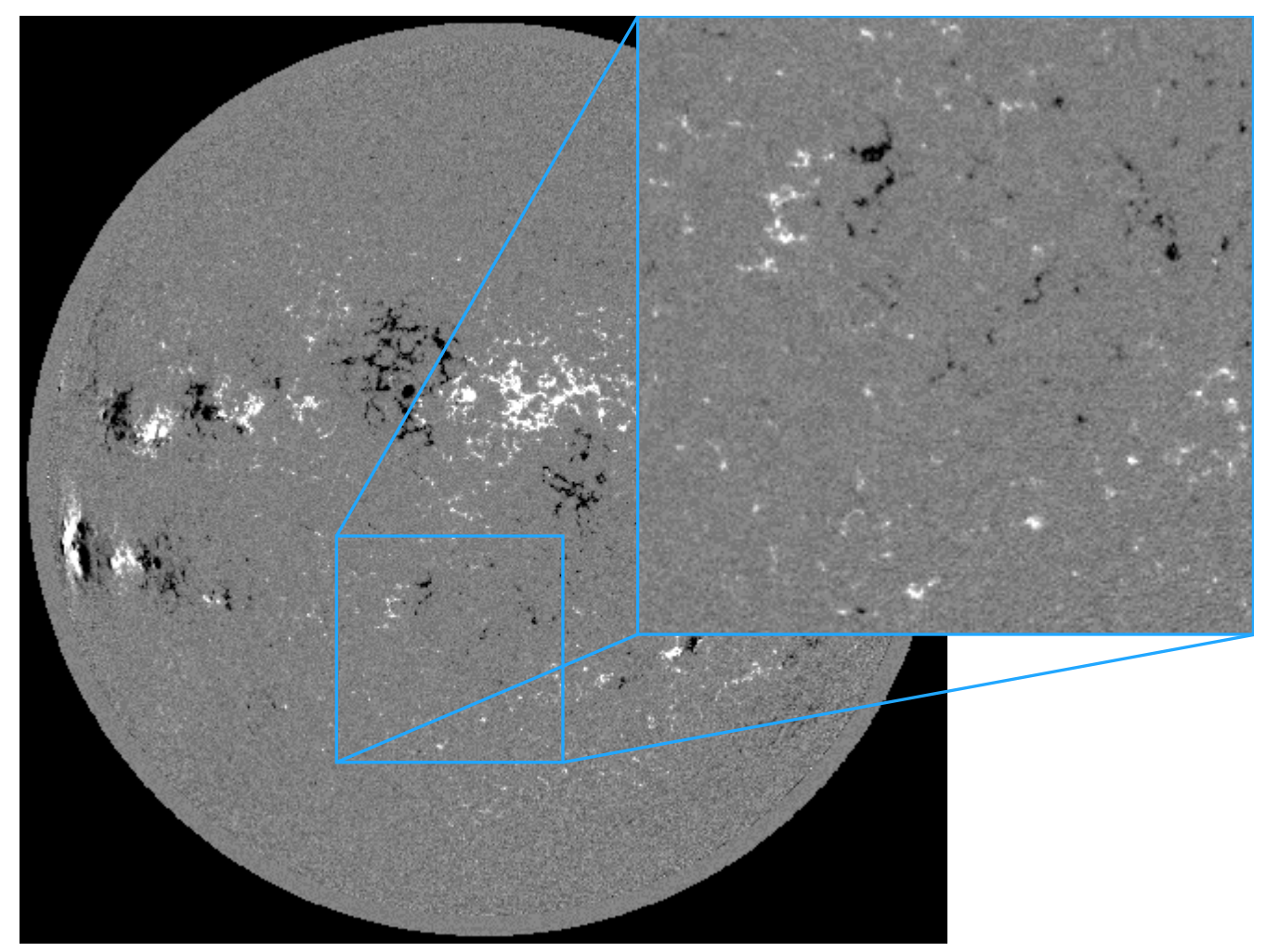

Figure 1.1: Full disk magnetogram of the Sun observed with the MDI instrument on board of the SOHO mission, in August 10, 2002. A relative quiet region of $10^{\prime} \times 10^{\prime}$ is enlarged showing the network.

magnetic. This is the so called quiet Sun. There is no clear definition on what the quiet Sun is. It is often used synonymously with the non-active Sun. In the present work it is denoted as the regions with no signal of polarization in synoptic magnetograms like in Fig. 1.1. These regions are also named internetwork (IN) as they correspond to the interior of the network cells. However, it is not clear how to distinguish between network and internetwork, as the presence of signal depends on the polarimetric sensitivity and on the spatial resolution of the magnetogram. With that uncertainty in mind, one can still state that the quiet Sun covers most of the solar surface, even during solar maximum. Figure 1.2 shows the areas covered by different active regions on the Sun. This area is very small compared with the area covered by the network, about $10 \%$, and seems to remain unchanged during the solar cycle (Harvey-Angle 1993).

Nevertheless, the quiet Sun is magnetically not so "quiet" as it was thought some decades ago. The absence of polarization signals, in magnetograms like in Fig. 1.2, is due to the low sensitivity and limited spatial resolution of the mea- 


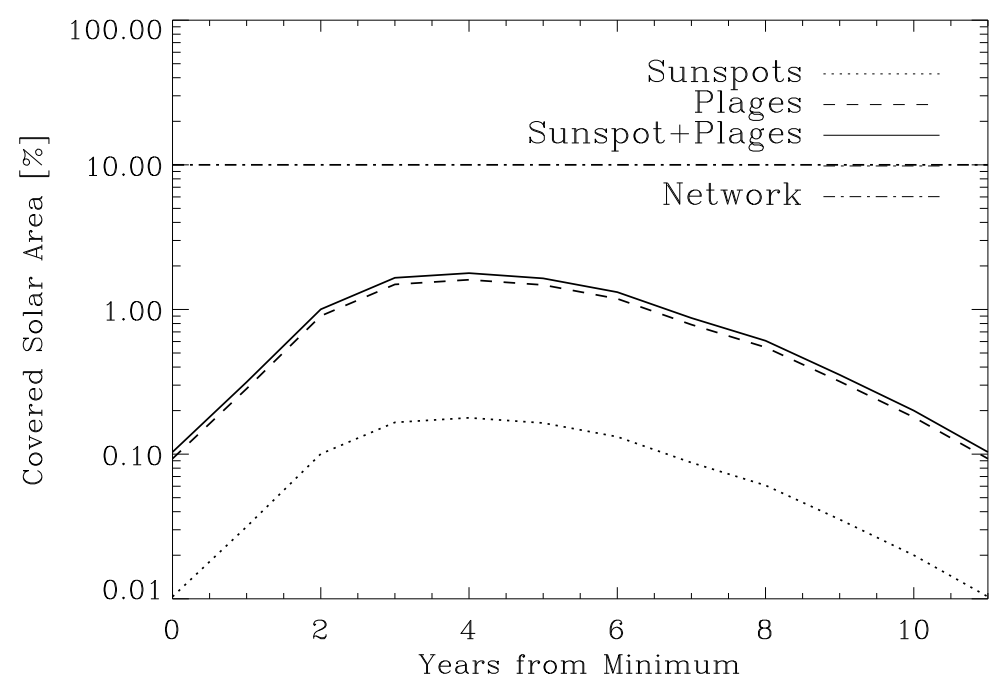

Figure 1.2: Variation of the filling factor of the network, sunspots and plage on the solar surface along the cycle. Even during maximum of activity most of the Sun is free from magnetic structures. Courtesy Jorge Sánchez Almeida (from Sánchez Almeida 2004)

suring instruments. Small, hitherto neglected contributions can become of high importance. The quiet Sun occupies most of the solar surface. In consequence, it may carry most of the solar magnetic flux (see, e.g., Stenflo 1982; Sánchez Almeida et al. 2003b). In that case, the quiet Sun can play a major role in the global solar magnetism which has not yet been taken into account.

In the last ten years, there has been an increasing interest in the study of the quiet Sun magnetism thanks to improvements in observational and diagnostic capabilities. On the one hand, when the sensitivity allows to measure magnetic fluxes of few $\mathrm{G}$ and the resolution is about $1^{\prime \prime}$ most of the solar surface turns magnetic (see, Grossmann-Doerth et al. 1996; Lin \& Rimmele 1999; Lites 2002). On the other hand, measurements based on the Hanle effect with chromospheric lines ${ }^{1}$ indicate the presence of a turbulent magnetic field everywhere in the quiet Sun (see Faurobert-Scholl et al. 1995; Bianda et al. 1998; Shchukina \& Trujillo Bueno 2003). Furthermore there are evidences of the presence of mixed polarities in 1" resolution elements (Sánchez Almeida \& Lites 2000, Lites 2002). This mixing of polarities reduces the polarization signals turning the spatial resolution to a crucial parameter in the quiet Sun observations, even more important than the polarimetric sensitivity.

\footnotetext{
${ }^{1}$ Lines formed in a higher layer above the photosphere, which is called chromosphere.
} 
Not much is known about the IN magnetic fields, their distribution or their origin. Magnetohydrodynamic simulations suggest a quiet Sun with magnetic structures at a small scale (see Cattaneo 1999; Vögler \& Schüssler 2003), with sizes much below the spatial resolution of today's telescopes (about $100 \mathrm{~km}$ ). A similar scenario is the MISMA concept (Sánchez Almeida et al. 1996; Sánchez Almeida 1997) as a MIcro-Structured Magnetic Atmosphere with optically thin flux tubes.

Nowadays there is a controversy on the distribution of the intrinsic magnetic fields of the IN. Observational studies based on infrared (IR) spectral lines find magnetic field strengths of sub-kG ${ }^{2}$ (see Lin 1995; Lin \& Rimmele 1999; Khomenko et al. 2003), while works using lines in the visible spectral range find $\mathrm{kG}$ fields (Grossmann-Doerth et al. 1996; Sigwarth et al. 1999; Sánchez Almeida \& Lites 2000; Socas-Navarro \& Sánchez Almeida 2002). Finally, studies using the Hanle effect find fields of tens of G (Bianda et al. 1999). Sánchez Almeida \& Lites (2000) suggested that this difference reflects the existence of a continuous distribution of field strengths from sub-kG to kG. Such contradiction in the measurements is due to different sensitivities of the lines to the magnetic field strength. While IR lines are more sensitive to sub-kG fields, visible lines detect $\mathrm{kG}$ fields (more details in Section 2.3)

This Thesis presents two new and exploratory observations performed to solve some problems sketched above. Chapter 2 gives a short introduction to polarimetry, including basic concepts of the Zeeman effect (the physical bases of the techniques applied here to measure magnetic field) and some details on the visible-IR controversy. Chapter 3 presents the first observations. It was possible with high spatial resolution spectropolarimetry to obtain magnetograms with $0{ }^{\prime \prime} .5$ resolution. It is demonstrated that high angular resolution allows to detect smallscale magnetic feature and the implications are discussed. Chapter 4 describes the second set of observations, simultaneous measurements of magnetic fields in the visible and IR spectral regions. The results can solve, at least in part, the visible-IR controversy. Finally, Chapter 5 presents a summary of the results and some final remarks.

\footnotetext{
${ }^{2}$ In the range below 1 kilogauss.
} 


\section{Chapter 2}

\section{Polarimetry}

Light can be defined as electromagnetic wave oscillations in one or more directions, perpendicular to the direction of propagation of the light. In general these oscillations can be decomposed into two waves in orthogonal directions in the plane perpendicular to the propagation:

$$
\begin{aligned}
& E_{1}(t)=\varepsilon_{1} e^{-i \omega t}=A_{1} e^{i\left(\phi_{1}-\omega t\right)} \\
& E_{2}(t)=\varepsilon_{2} e^{-i \omega t}=A_{2} e^{i\left(\phi_{2}-\omega t\right)}
\end{aligned}
$$

with $A_{j}$ the amplitudes and $\phi_{j}$ the phases of the waves. These constants are contained in the complex constants $\varepsilon_{j}$ and combinations of them include all kind of polarized states of the light.

Polarization of light is usually described by the Stokes parameters. They are defined as different bilinear products formed from two components:

$$
\begin{aligned}
& I=k\left(<\varepsilon_{1}^{*} \varepsilon_{1}>+<\varepsilon_{2}^{*} \varepsilon_{2}>\right) \\
& Q=k\left(<\varepsilon_{1}^{*} \varepsilon_{1}>-<\varepsilon_{2}^{*} \varepsilon_{2}>\right) \\
& U=k\left(<\varepsilon_{1}^{*} \varepsilon_{2}>+<\varepsilon_{2}^{*} \varepsilon_{1}>\right) \\
& V=i k\left(<\varepsilon_{1}^{*} \varepsilon_{2}>-<\varepsilon_{2}^{*} \varepsilon_{1}>\right),
\end{aligned}
$$

where "*" means complex conjugate, $\langle\cdots\rangle$ denotes average over a statistical sample, and $k$ is a constant. $I$ is the total intensity, $Q$ is the linear polarization parallel to one freely chosen axis, $U$ is the linear polarization under $45^{\circ}$ with respect to this axis, and $V$ is the circular polarization. More details can be found in Chandrasekhar (1950).

Cameras can only measure intensity (Stokes $I$ ). To measure the other parameters one needs an analyzer of polarization. There is a variety of different instruments for that purpose. The most used one in solar physics consists basically of a retarder and a linear polarizer. Combinations of the parameters in such optical elements can transform the excess of polarization (circular or linear) from the incoming light into intensity that can illuminate a detector. By modulation 
of retardance and direction of the linear polarizer it is possible to obtain right and left handed circularly polarized light, or linear polarization in any direction. These are linear combinations of the four Stokes parameters, so from at least 4 orthogonal quantities of this kind one can obtain the full Stokes vector.

\subsection{Zeeman effect}

Spectral lines are produced by transitions between two discrete levels of an atom (or ion, or molecule). The energy difference between the levels gives the photon frequency, absorbed or emitted by the atom in this transition. In the solar atmosphere, such processes reduce the mean free path of the photons in this frequency, so the photons come from upper layers of the photosphere. These layers are cooler than the deeper ones. Thus the plasma emits less light which results in spectra with absorption lines at the wavelengths corresponding to the transitions.

Usually, one or both atomic levels are degenerated, i.e. the energy does not depend on the orientation of the total angular momentum (described by the quantum number $\mathrm{J}$ ). In the presence of a magnetic field this degeneracy of the levels can be broken, which allows to distinguish between the sublevels and produces a splitting of the spectral line. This is known as the Zeeman effect, and a simple case is the triplet shown in Fig. 2.1. A classical explanation of this phenomenon is the Larmor theorem. According to it, the oscillation of an atom in the presence of a magnetic

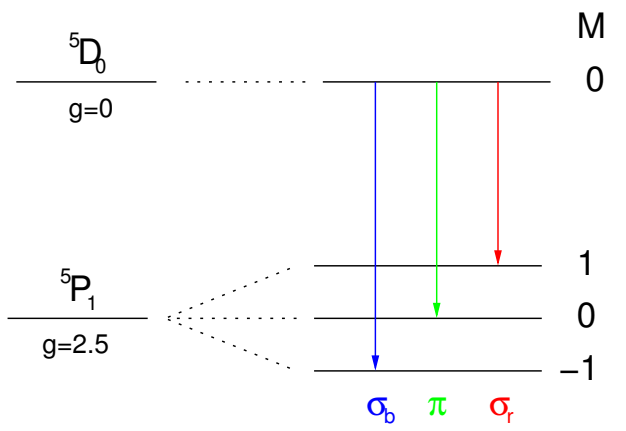

field can be decomposed in three independent components, two circular and one linear (see Fig. 2.2). The linear component $(\pi)$ remains unperturbed, but the circular components oscillate in a plane perpendicular to the linear component, with frequencies shifted by $\pm \nu_{L}$ (the sign depends on the direction of the oscillation) from the central frequency of the transition, $\nu_{0}$. This shift is known as Larmor frequency and is defined as:

$$
\nu_{L}=\frac{e_{0} B}{4 \pi m_{e} c},
$$

with $e_{0}=1.602 \times 10^{-19} \mathrm{C}$ and $m_{e}=9.109 \times 10^{-31} \mathrm{~kg}$, the electric charge and mass of the electron, respectively, $c=2.9979 \times 10^{8} \mathrm{~m} / \mathrm{s}$ the velocity of light in 


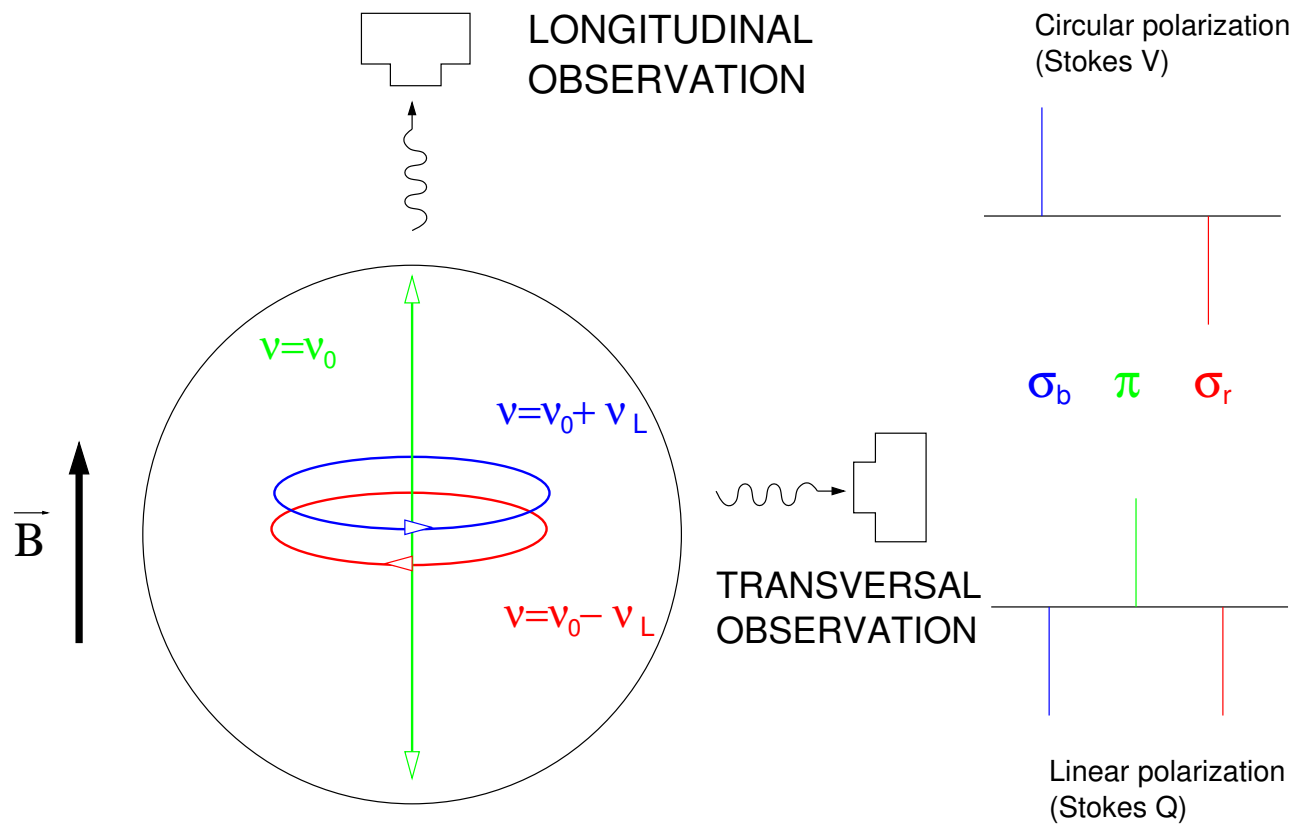

Figure 2.2: Classical atomic model in the presence of a magnetic field given by Larmor theorem. The frequencies of the circular components $(\sigma)$ are shifted by $\pm \nu_{L}$ while the longitudinal component $(\pi)$ keeps unperturbed.

vacuum, and $B$ the magnetic field responsible for the splitting.

Taking the example of the triplet, applied to this classical model, it is possible to understand what happens in terms of polarization. If the detector is illuminated by photons in the direction of the axis of the $\pi$ component (longitudinal observation in Fig. 2.2) it is possible to detect circular polarization in the two $\sigma$ components. As Stokes $V$ is positive for counterclockwise circular polarization there is positive $V$ at the blue-shifted frequency, and negative $V$ at the red-shifted frequency, and no detection of linear polarization. A transversal observation will give no circular polarization and positive linear polarization for the $\pi$ component and negative for the $\sigma$ components $^{1}$.

In general an explanation by quantum theory is required. For quantum numbers of the upper and lower level $\left(\mathrm{J}, \mathrm{J}^{\prime}\right)$ the $\pi$ components are transitions with $\Delta \mathrm{M}=0$ and for the $\sigma$ components holds $\Delta \mathrm{M}= \pm 1$. The splitting of each sublevel is $\nu_{M}=$

\footnotetext{
${ }^{1}$ when Stokes $Q$ is defined positive along the axis of the $\pi$ component
} 
$g M \nu_{L}$ with the Landé factor $g$. For levels with L-S coupling one has:

$$
g=\frac{3}{2}+\frac{S(S+1)-L(L+1)}{2 J(J+1)} .
$$

The splitting between the different components of the multiplet is $\nu_{M \rightarrow M^{\prime}}=$ $\left(g M-g^{\prime} M^{\prime}\right) \nu_{L}$. The effective Landé factor which parametrizes the sensitivity of the transition to the magnetic field, can be defined as

$$
g_{L}=\frac{1}{2}\left(g+g^{\prime}\right)+\frac{1}{4}\left(g-g^{\prime}\right)\left[J(J+1)-J^{\prime}\left(J^{\prime}+1\right)\right] .
$$

with $g$ annd $g^{\prime}$ thee Landé factor of the upper and lower level, respectively. In the case of a non triplet trasition Eq. 2.5 considers the center of gravity of the $\sigma$-components which are located at $\pm g_{L} \nu_{L}$ from the central frequency.

\subsection{Radiative Transfer}

To study spectral lines from a stellar atmosphere it is necessary to introduce the theory of radiative transfer. The introduction of a magnetic field and the study of the polarization complicates the Radiative Transfer Equation (RTE), as there are four parameters (Stokes $I, Q, U$ and $V$ ) giving information about the properties of the atmosphere. The RTE for polarized light in local thermodynamic equilibrium (LTE) becomes a vectorial equation (Landi Degl'Innocenty 1992):

$$
\frac{d}{d \tau}\left(\begin{array}{c}
I \\
Q \\
U \\
V
\end{array}\right)=\left(\begin{array}{cccc}
\eta_{I} & \eta_{Q} & \eta_{U} & \eta_{V} \\
\eta_{Q} & \eta_{I} & \rho_{V} & -\rho_{U} \\
\eta_{U} & -\rho_{V} & \eta_{I} & \rho_{Q} \\
\eta_{V} & \rho_{U} & -\rho_{Q} & \eta_{I}
\end{array}\right)\left(\begin{array}{c}
I-B_{T} \\
Q \\
U \\
V
\end{array}\right)
$$

where $d \tau=-\kappa_{c} d z$ is the optical depth measured in the continuum close to the line. The optical depth is directly related with the geometrical depth $(d z)$ through the continuum absorption coefficient $\left(\kappa_{c}\right)$. Under LTE approximation the emission is written as the Planck function $\left(B_{T}\right)$. The diagonal terms in the matrix $\left(\eta_{I}\right)$ are the absorption terms, while dichroic terms $\left(\eta_{Q}, \eta_{U}\right.$ and $\left.\eta_{V}\right)$ relate the intensity with the polarized states, and the dispersion terms $\left(\rho_{Q}, \rho_{U}\right.$ and $\left.\rho_{V}\right)$ are the links among the different polarized states. Equation 2.6 must be solved for each wavelength independently.

There are many ways and approximations to solve this equation depending on the level of sophistication required. For example, a commonly used approximation is the assumption of a Milne-Eddington atmosphere (see Section 4.5) providing an analytical solution to Eq. 2.6. Another way is to solve the full equation numerically under the assumption of a more complex atmosphere (like MISMA, see Section 4.6). 

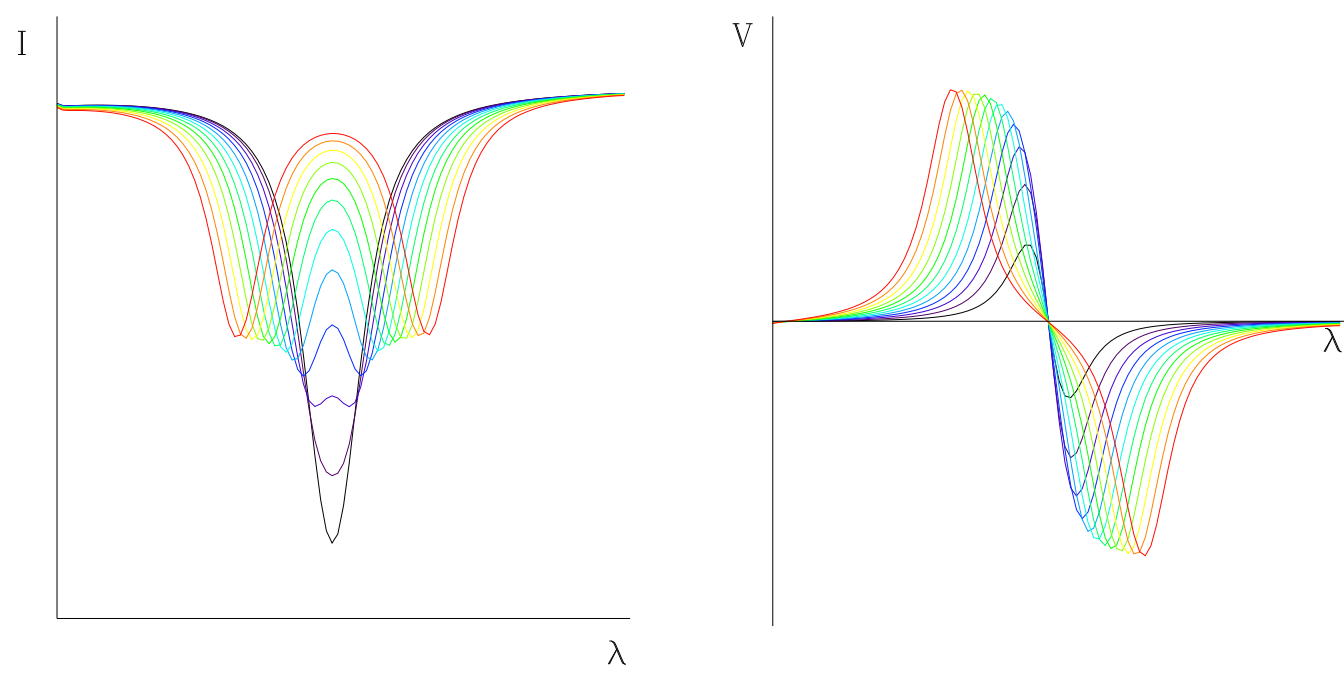

Figure 2.3: Example of Stokes $I$ and Stokes $V$ profiles of Fe I $\lambda 6302.5 \AA$ synthesized under ME approximation with longitudinal magnetic fields from $300 \mathrm{G}$ (black) to $3.6 \mathrm{kG}$ (red). Magnetic saturation starts at $1.5 \mathrm{kG}$ (cyan).

An illustrative example of how Stokes profiles look like is presented in Fig. 2.3. These profiles were computed for a Milne-Eddington atmosphere embedded in different longitudinal magnetic fields with field strengths from $300 \mathrm{G}$ to $3.6 \mathrm{kG}$ (with steps of $300 \mathrm{G})^{2}$. This is the extreme case of Longitudinal Observation in Fig. 2.2 (no linear polarization) and the Stokes $V$ profiles show the two $\sigma$ components with a splitting growing with the magnetic field strength. However the components are not entirely separated for weak magnetic fields (black-blue). This is due to the broadening of the spectral line, caused by natural broadening of the transition and mainly by the Doppler broadening $\left(\Delta \lambda_{D}\right)$ due to the stochastic thermal velocities $\left(\omega_{T}\right)$ :

$$
\Delta \lambda_{D}=\lambda_{0} \frac{\omega_{T}}{c},
$$

with $\lambda_{0}$ the central wavelength of the line. In this case the increase of the separation of the components with magnetic field strength increases the polarization signal, but there is no growth of the splitting of the maxima of the lobes. This weak field regime is fulfilled when the Zeeman splitting is much smaller than the Doppler broadening $\left(\Delta \lambda_{B}<<\Delta \lambda_{D}\right)$.

In the case of strong fields (green-red) the increase of the polarization signal

\footnotetext{
${ }^{2}$ This work uses the Gauß system as is still common in solar physics. Furthermore, the diamagnetic constant $\mu$ is always 1 and the magnetic field strength is used synonymously with magnetic flux density. $1 \mathrm{G}$ (Gauss) $=10^{-4} \mathrm{~T}$ (Tesla).
} 
is smaller and the splitting of the lobes dominates. The line is magnetically saturated and the splitting can be defined as

$$
\Delta \lambda_{B}=\frac{e_{0}}{4 \pi m_{e} c} \lambda^{2} g_{L} B .
$$

The last two sections were meant as a basic sketch of the Zeeman effect and the RTE for polarized light. More detailed explanations can be found in Landi Degl'Innocenti (1992).

\subsection{The controversy on spectral lines in the vis- ible and the infrared spectral ranges}

The contradictory measurements of intrinsic magnetic fields in the quiet Sun obtained from observations in IR and visible lines can be explained with a continuous distribution of magnetic fields where $\mathrm{kG}$ and sub- $\mathrm{kG}$ fields co-exist in the observational resolution elements (see Section 1.2).

Sánchez Almeida \& Lites (2000) put forward a mechanism that biases the IR observations toward sub-kG fields, caused by a vertical gradient of the field strength. Magnetic sensitivity goes with the Landé factor but also with the square of the wavelength (see Eq. 2.8), implying that IR lines are much more sensitive to magnetic fields than visible lines. In the case of the Fe I $\lambda 15648 \AA$ line (with $g_{L}=3$ ) the magnetic saturation occurs at sub-kG fields (about $400 \mathrm{G}$ ), thus $\mathrm{kG}$ fields result in clearly splitted components. On the one hand, a distribution of different, yet strong, fields will produce polarization signals always at different wavelengths. These signals will be spread over a wide range of wavelength and will dilute the polarization along the profile. On the other hand different sub-kG fields will produce the polarization signals at the same wavelengths (in the weak field regime), resulting in a significant level of polarization.

Fig. 2.4 shows, with a basic example, how independent observations in IR and visible spectral lines can result in sub-kG and $\mathrm{kG}$ fields, respectively (as in Socas-Navarro \& Sánchez Almeida 2003). Imagine an atmosphere with two longitudinal magnetic fields of $400 \mathrm{G}$ (weak component) and $1600 \mathrm{G}$ (strong component) and filling factors of 0.8 and 0.2 respectively, resulting in the same magnetic flux in both components. A synthesis was done for the lines used in IN (Intranetwork) observations, the IR Fe I $\lambda 15648 \AA$ and the visible pair Fe I $\lambda 6301.5 \AA$ and Fe I $\lambda 6302.5 \AA$. In the IR, both components (see upper panels in Fig. 2.4) have a similar level of polarization, since the IR line is starting to saturate at the field strength of the weak component. For the visible pair, the strong component produces much more $V$ signal than the weak component. 

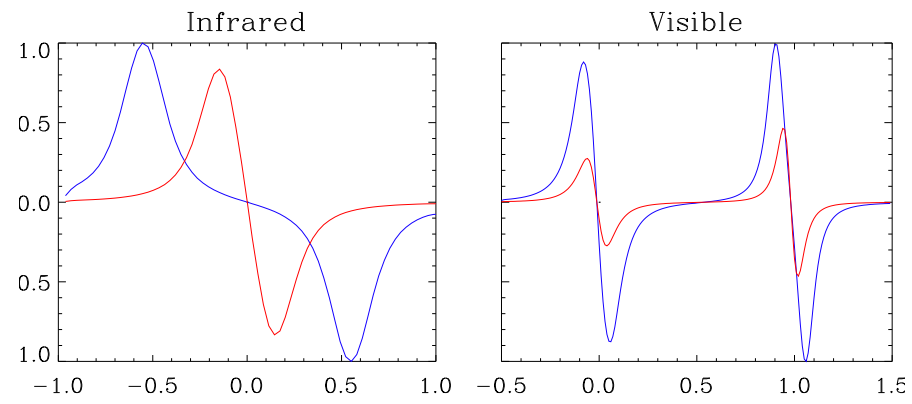

Figure 2.4: Synthesis of Stokes $V$, for a two-magneticcomponent model, of the infrared line Fe I $\lambda 15648 \AA$ (left), and the visible pair Fe I $\lambda 6301.5 \AA$ and Fe I $\lambda 6302.5 \AA$ (right). The upper panels show profiles for individual components:

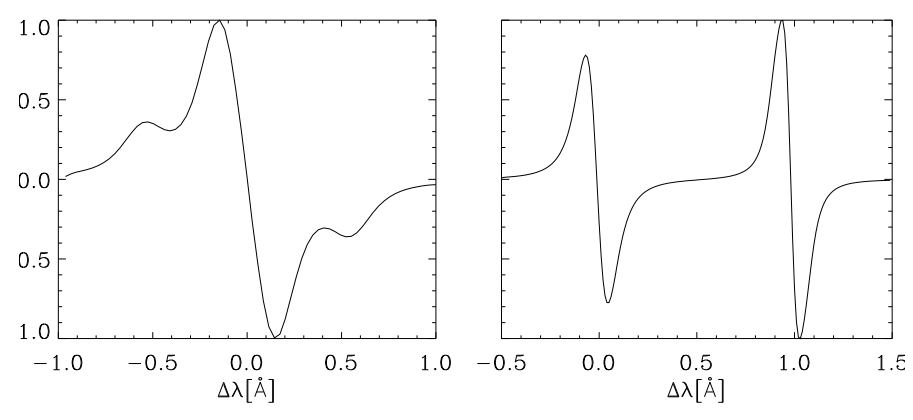
weak $\left(B_{w}=400 \mathrm{G}\right.$ in red) and strong $\left(B_{s}=1600 \mathrm{G}\right.$ in blue) magnetic fields with filling factor $\alpha=1$. The bottom panels show the result of adding both components, with $\alpha_{w}=0.8$ and $\alpha_{s}=0.2$. Profiles are normalized to the maximum in each panel.

The consequence of adding both components with adopted filling factors is displayed in the bottom panels of Fig. 2.4. The IR profile shows essentially the weak field profile with small additional lobes from the strong field component. In real observations, this component is hidden by the noise, and the use of the splitting to measure the field strength yields to sub-kG fields (as done by Lin 1995 and Khomenko et al. 2003). Meanwhile the visible profile does not at all look like a two-component profile. The use of the ratio method

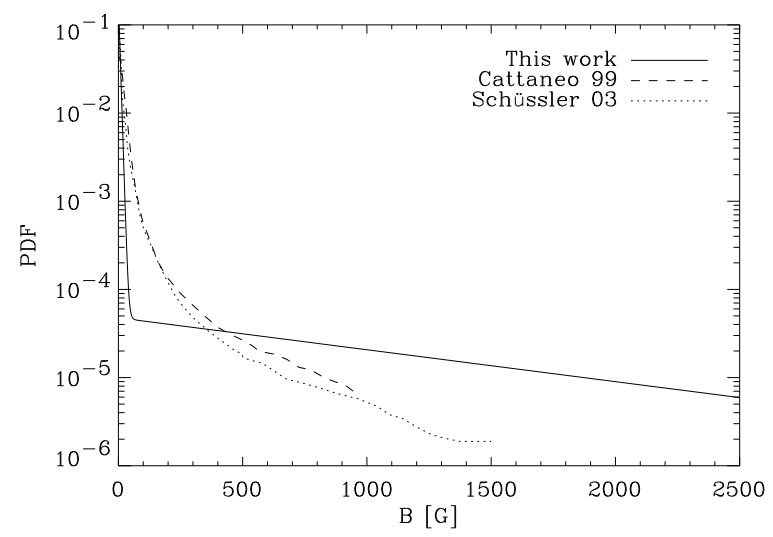

Figure 2.5: Probability Density Function (PDF) from Socas-Navarro \& Sánchez Almeida (2003). (see Section 3.4.1) or a simple Milne-Eddington inversion (see Section 4.5) leads to $\mathrm{kG}$ magnetic fields. In this case the ME inversion result is $1200 \mathrm{G}$.

Socas-Navarro \& Sánchez Almeida (2003) also explain this bias in IR observations with horizontal gradients of magnetic fields. They consider an atmosphere having a distribution of magnetic field strengths with weak fields filling a larger area than strong fields but with a not too large difference, so the strong fields carry 

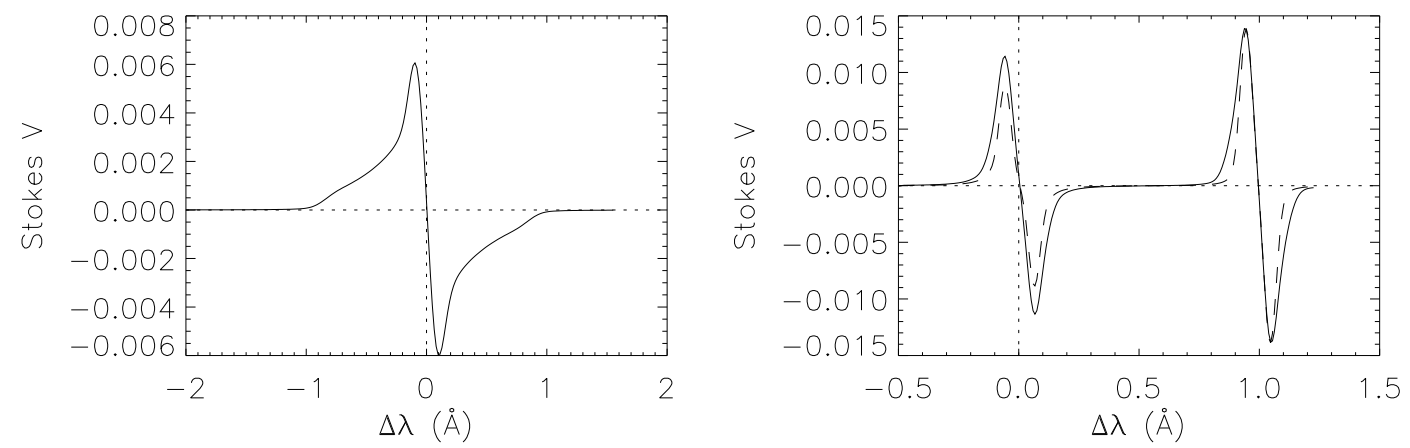

Figure 2.6: Synthesis from PDF in Fig. 2.5 for the IR Fe I $\lambda 15648 \AA$ (left) and for the visible pair Fe I $\lambda 6301.5 \AA$ and Fe I $\lambda 6302.5 \AA$ (right). Courtesy Hector Socas-Navarro (High Altitude Observatory).

most of the magnetic flux. Fig. 2.5 show the probability density function (PDF) with the occupation of each magnetic field strength according to such continuous distributions. The synthesis for the same IR and visible lines is done resulting on the left and right panel of Fig. 2.6. Now the IR line has two clear lobes of weak fields and a tail corresponding to the spread of the strong components. A one component inversion of such profiles yields a magnetic field of $250 \mathrm{G}$ for the IR line and $1100 \mathrm{G}$ for the visible pair. 


\section{Chapter 3}

\section{High spatial resolution polarimetry}

\subsection{Speckle reconstruction}

To improve the spatial resolution of images is one of the main goals in observational astrophysics. The limitation for ground-based telescopes is the presence of our atmosphere, a turbulent gas affecting the path of the light, deforming the wavefronts and degrading the images. Nowadays, there are three different techniques in use in solar physics:

- Adaptive optics. It is a real time technique to improve the images at the moment of the gathering, correcting the wavefront of the light with deformable mirrors (i.e. see Tyson 1998).

- Phase diversity. It is a post facto technique ${ }^{1}$ based on the use of a focussed image and a simultaneous taken defocussed one (with known defocussing).

- Speckle reconstruction. The technique used in this work, it is also a post facto application and is based on statistics of many speckle images.

A speckle image is taken with an exposure time shorter than the timescale of the variations in the atmosphere such that the instantaneous effect of the atmosphere is frozen in the image. This time is of the order of typically 20-50 ms. Figure 3.1 shows a time sequence of speckle images of a quasi point source, the star Betelgeuse. Each image is not just a point but an inhomogeneous distribution of specks due to very complicated refraction effects in the atmosphere. This is the characteristic of a speckle image. The effect produces the famous

\footnotetext{
${ }^{1}$ Technique applied to images already observed
} 

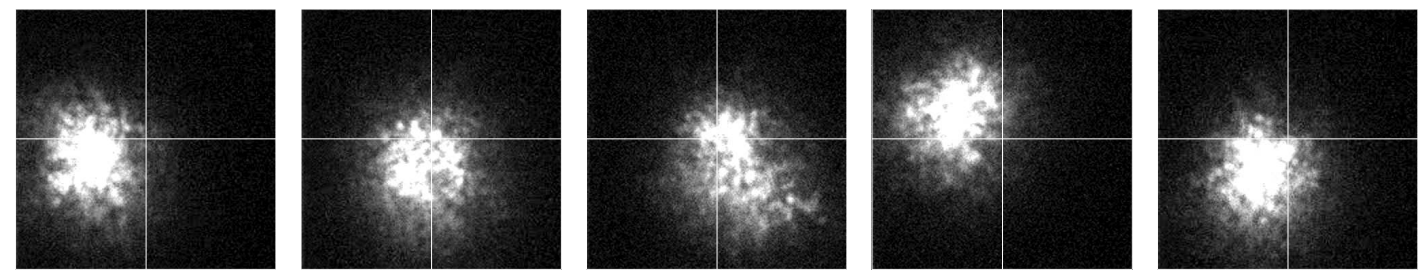

Figure 3.1: Different snapshots of speckle images from Betelgeuse with a central cross to see the motion. They were taken by the Applied Optics Group (Imperial College) with the Herschel 4.2-m Telescope, at the Observatorio del Roque de los Muchachos on La Palma (Spain).

twinkle of the stars. The distribution of the specks changes during time and also moves globally. The result of a summation of many images like in the sequence of Fig. 3.1 is the characteristic distribution for a long exposure image, a kind of Gaussian with a FWHM equal to the seeing parameter.

An astrophysical image can be defined as

$$
i(\vec{x})=o(\vec{x}) \star s(\vec{x}),
$$

with $i(\vec{x})$ the image measured on the camera. This image is the convolution of the true image $(o(\vec{x}))$ with the Point Spread Function (PSF, $s(\vec{x}))$ which contains the effects of the telescope and the atmosphere. Such a convolution corresponds to a multiplication in Fourier space where the average of $N$ images is

$$
\frac{1}{N} \sum_{i=1}^{N} I_{i}(\vec{u})=O(\vec{u}) \cdot \frac{1}{N} \sum_{i=1}^{N} S_{i}(\vec{u}) .
$$

The left side of Eq. 3.2 corresponds to a long exposure image, with typical degradation. This image is equal to the Fourier Transform of the true image $O$ multiplied with the summation of the instantaneous Optical Transfer Functions $(\mathrm{OTF}=F T\{\mathrm{PSF}\}) . \quad$ It is in this summation of the OTF, where the spatial resolution is lost. Changes of the atmosphere introduce varying phases in each instantaneous complex OTF. The averaging of the OTFs with different phases produces cancellations in the complex amplitude affecting most of the high spatial frequencies. The result is an image with a resolution corresponding to the so called seeing conditions, while the information on higher frequencies, which is still present in the single exposures, is lost.

To recover this information, Labeyrie (1970) proposed the following method. To avoid the cancellation, the individual terms in Eq. 3.2 are replaced by their 
square modulus:

$$
\frac{1}{N} \sum_{i=1}^{N}\left|I_{i}(\vec{u})\right|^{2}=|O(\vec{u})|^{2} \cdot \frac{1}{N} \sum_{i=1}^{N}\left|S_{i}(\vec{u})\right|^{2} .
$$

The average of the square of the instantaneous OTFs is called the Speckle Transfer Function (STF). This function is easy to obtain in the case of stars, i.e. of point sources. For a real point source we now $O$. In solar observations it is not so evident to retrieve the STF, since the images come from an extended source with no possibility to observe simultaneously a point source. However, it is possible to model this STF, with dependence only on the seeing conditions. It can be evaluated with the Fried parameter $\mathrm{r}_{0}$ (Korff 1973) which must be known, i.e. be determined with the spectral ratio method by von der Lühe (1984). Such a calculation needs a large number of speckle images (of the order of 100). They must be taken faster than the time scale of the variations of the structures which are observed. This depends on the resolution one wants to achieve (e.g. for 0.2 resolution the time should be about 1 minute for solar granulation).

With the STF and Eq. 3.3 it is possible to obtain $|O|^{2}$. However this gives only the amplitude of the image in Fourier space. A further method is needed to obtain the phases at each frequency. The speckle reconstruction code used in this work (see de Boer 1992 and de Boer 1996) uses the speckle masking method proposed by Weigelt (1977) and Weigelt \& Wirnitzer (1983). It is based on the bispectrum defined as

$$
B S(i, j, k, l)=\langle I(i, j) \cdot I(k, l) \cdot I(-i-k,-j-l)\rangle
$$

with $(i, j, k, l=-N / 2, \ldots, N / 2)$ and the brackets indicating averaging over the Fourier transform of the speckle images. It can be shown (e.g. de Boer 1993) that the averaging over sufficiently many OTFs contained in Eq. 3.4 yields a purely real, non-zero function. The bispectrum thus contains only the object phases. The bispectrum is a four-dimensional array containing multiplications with different displacements of the images in Fourier space. So it has encoded the phases as differences which can be recovered with the phase closure equation

$$
e^{\mathbf{i} \phi(i+k, j+l)}=e^{\mathbf{i} \phi(i, j)} e^{\mathbf{i} \phi(k, l)} e^{-\mathbf{i} \Phi(i, j, k, l)},
$$

where $\Phi(i, j, k, l)$ represents the phases of the bispectrum. This is a recursive equation and from few starting conditions it is possible to obtain all the phases. The higher the frequency the more equations are available. An average of the results is used for each point in Fourier space. The starting conditions are

$$
\phi(0,0)=0 ; \phi(0,1) ; \phi(1,0) .
$$

The latter two phases are obtained from the original data since the phases at low frequencies are well known. 


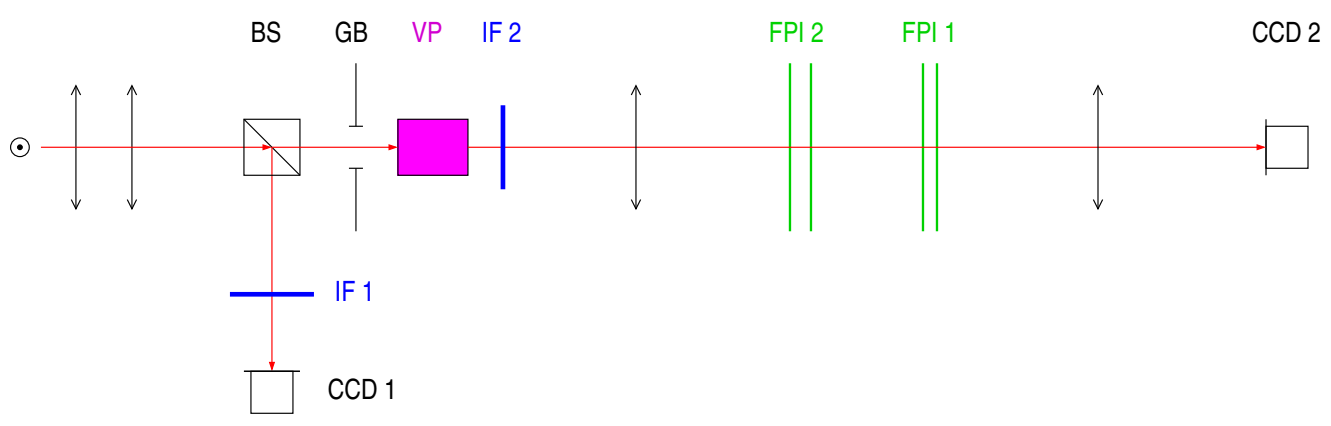

Figure 3.2: Schematical setup of the Göttingen FPI.

\subsection{Observations: FPI}

To obtain two-dimensional (2D) spectroscopic data one can use a very narrowband tunable filter and records images at various wavelengths by scanning through spectral lines. However, to improve the spatial resolution of such kind of data, it is not possible to apply directly the speckle reconstruction technique, due to the need of many images and many photons to ensure high resolution. Narrow filters decrease drastically the number of photons and the instrumental limitations do not allow to take many images per wavelength position. The solution to this problem is to take broad-band images simultaneously with the narrow-band ones. The broad-band images can be obtained using a broad filter (100 $\AA$ ), so they can be reconstructed with speckle techniques. Using this reconstructed and each instantaneous image it is possible to obtain the instantaneous OTF for each individual narrow-band image (see Section 3.3.1)

An instrument for such observations was built in the decade of nineties by the solar group at the Sternwarte in Göttingen. It is the Göttingen Fabry-Perot Interferometer (FPI; Bendlin et al. 1993; Bendlin 1993; Bendlin \& Volkmer 1995) mounted at the German Vacuum Tower Telescope (VTT) of the Observatorio del Teide (Tenerife, Spain).

The observations were obtained in April 29, 2002, under good seeing conditions $\left(\mathrm{r}_{0}=14 \mathrm{~cm}\right)$ using the setup displayed in Fig. $3.2^{2}$. The target was a very quiet Sun region at disk center, selected using G-band video images to avoid network. The instrument uses two CCD detectors taking simultaneously images with an exposure time of $30 \mathrm{~ms}$. The CCDs are identical, with $384 \times 286$ pixels, with a pixel size corresponding to $0^{\prime \prime} 1 \times 0^{\prime \prime} 1$. Light coming from the Sun is split into two beams with a beam splitter (BS). About $5 \%$ of the light goes to the broad-

\footnotetext{
${ }^{2}$ This is the basic version. A more complete one is given in Koschinsky 2001.
} 


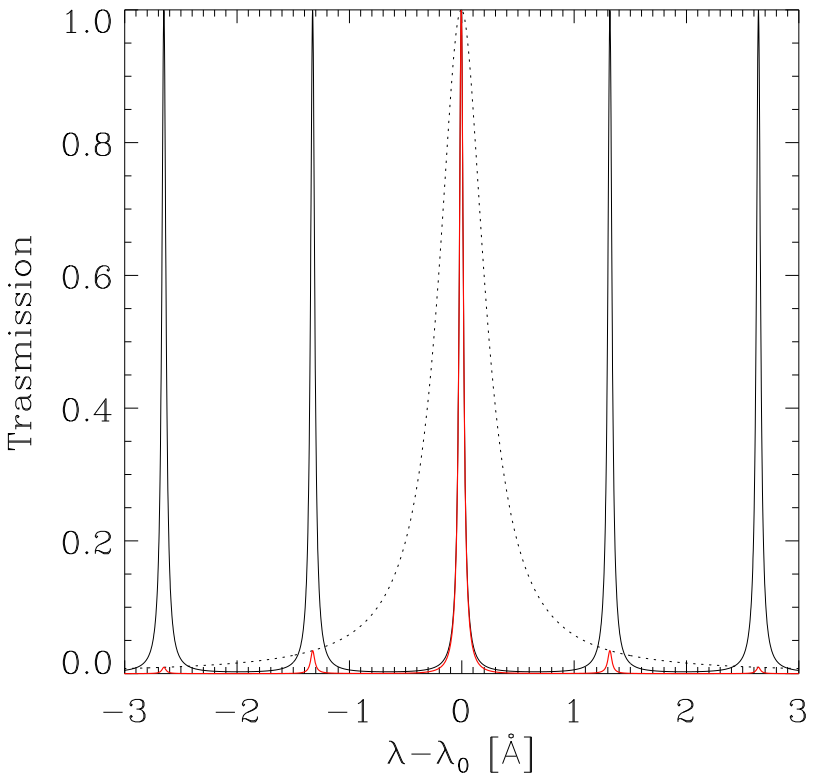

Figure 3.3: Transmission functions of the FPI spectrometer (red) and for each individual Fabry-Perot interferometer (black lines, dotted for the broad one and solid for the narrow one).

band detector (CCD 1), after an interference filter (IF 1) centered at $6300 \AA$ with band-pass of the order of $100 \AA$. This camera gathers 150 images to be reconstructed by speckle techniques (described in Section 3.1). The remaining $95 \%$ of the light is used to scan the spectral lines. This beam is split into the left and right circularly polarized components of the light by means of a Stokes $V$ analyzer (VP, see Koschinsky 2001). Each component illuminates different areas of the narrow-band detector (CCD 2), after passing trough the two Fabry-Perot etalons. A synchronized change of the distance of the plates of these two etalons (FPI 1 \& FPI 2) has the effect of a tunable filter which is used to scan along wavelength. Figure 3.3 shows the transmission function of each individual etalon (black) and of the combination of both. FPI 2 gives a narrow transmission and will define the spectral resolution $(44 \mathrm{~m} \AA$ ), while FPI 1 is broader and removes the other orders of FPI 2. In the same way, an interference filter centered at $6301 \AA$, with a FWHM of $8 \AA$ (IF 2) removes the other orders of FPI 1.

The spectral lines observed are the magnetically sensitive pair of iron lines Fe I $\lambda 6301.5 \AA$ (with a Landé factor $\left.g_{L}=1.67\right)$ and Fe I $\lambda 6302.5 \AA\left(g_{L}=2.5\right)$, and the telluric line $\mathrm{O}_{2} \lambda 6302.8 \AA$ (Fig. 3.4 shows the observed spectral range). The step size between wavelength positions was set to $31.8 \mathrm{~m} \AA$. The spectral scans consist of 5 wavelength positions within the telluric line (2 images per position) and 14 positions ( 5 images per position) within each of the iron lines. The time needed for scanning the lines is $35 \mathrm{~s}$, and the system needs $15 \mathrm{~s}$ to store all the 150 images obtained from both CCDs onto hard disk. The data gathered consist of a time series of 17 min duration. Within that time, 20 spectral scans were taken again simultaneously with integrated light. 
Figure 3.4: Comparison of the effect of the FPI on the Stokes $I$ profile from the flat field data (circles) with FTS spectra before (red) and after (black) convolution with the FPI transmission function

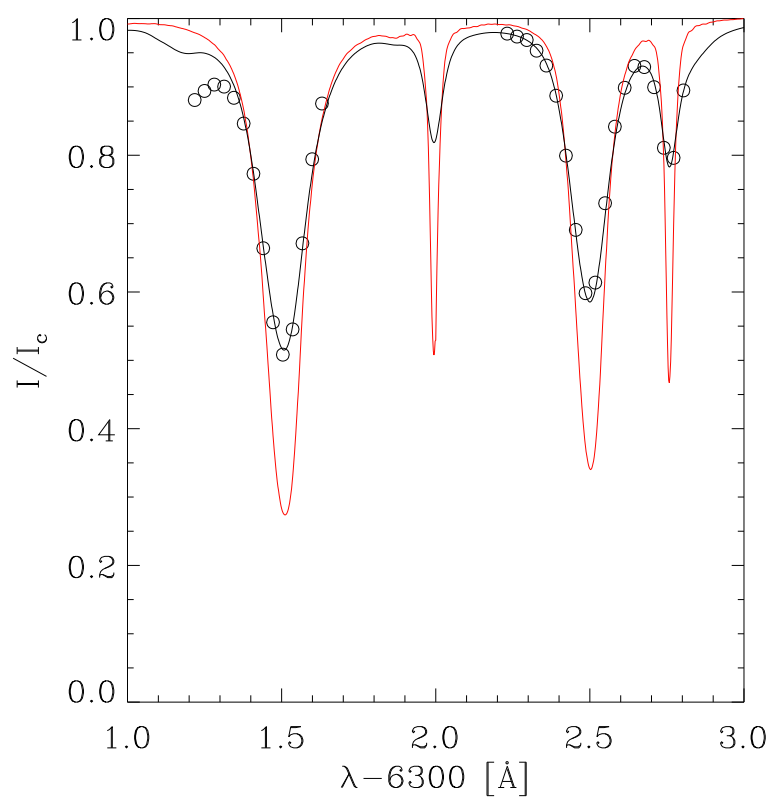

It is a good exercise to check how much false light from the wings of the transmission functions is affecting the line profiles. The data is compared with a solar spectrogram of high spectral resolution. Figure 3.4 shows the FTS data (Fourier Transform Spectrometer, Brault \& Neckel 1987) before (red) and after (black) convolution with the transmission function of the FPI. One can find the good agreement with the observed spectra (circles).

In addition to the data gathered for the reconstruction, it is highly important to measure dark current fields, flat fields, and continuum images. These images are used in the reduction of the data before the restoration, and also in some stages of the narrow-band reconstruction. Dark frames are images with no illumination on the CCD, in order to measure the counts produced by the temperature of the chip, depending only on the exposure time. Thus, offset must be subtracted fom all images. It has a tiny slope in the reading direction of the CCD. To avoid the adding of noise to the data these dark frames are fit to a surface of first order. Flat fields are images with homogeneous illumination from the Sun on the CCD. Such frames were obtained with by averaging images taken with moving telescope pointing on a quiet Sun region, or with defocussed images. They correct for the different behaviour of pixels over the FOV of the chip. Finally, continuum images are measured with CCD 2 with light from a halogen lamp passing through the spectrometer. This light has no dependence on wavelength, i. e. contains no spectral lines, thus it can be used to correct for the wavelength dependence of the transmission of the optical setup (mainly due to IF 2 in Fig.3.2). The use of such data is standard and will not be described further (but see Koschinsky 2001; Janßen 2003; Okunev 2004). 


\subsection{Data Analysis}

\subsubsection{Narrow-band reconstruction}

Reconstruction of the narrow-band data obtained with CCD 2 was carried out with a modification of the code by Janßen (2003). This code works with the method by Keller \& von der Lühe (1992) based on obtaining the instantaneous OTFs from the broad-band data. In Fourier space the CCD 2 images at each individual wavelength position are

$$
I_{n, j}=S_{j} \cdot \hat{O}_{n},
$$

with $j=1, \ldots, M$, and $\mathrm{M}$ the number of images per position. The respective images in broad-band are

$$
I_{b, j}=S_{j} \cdot \hat{O}_{b} .
$$

Now $\hat{O}_{n}$ and $\hat{O}_{b}$ are estimates of the narrow-band (unknown) and broad-band (already reconstructed) images respectively. Applying a least-square calculation to the $M$ observations in Eq. 3.7 one obtains

$$
\hat{O}_{n}=\frac{\sum_{j} I_{n, j} S_{j}^{*}}{\sum\left|S_{j}\right|^{2}}
$$

Introducing the instantaneous OTFs from Eq. 3.8 yields the final formula

$$
\hat{O}_{n}=H \frac{\sum_{j} I_{n, j} I_{b, j}^{*}}{\sum_{j}\left|I_{b, j}\right|^{2}} \hat{O}_{b},
$$

where a noise filter $H$ was introduced. The latter can be calculated as an optimum filter taking into account the level of noise in the flat field images and the corresponding light levels (see Krieg et al. 1999, or Koschinsky et al. 2001). However, this filter changes with the wavelength possition as there are less counts in the core of the lines, the cutoff there occurs at smaller frequency. A change in resolution through the line profile is unacceptable in studies of small-scale structures like the magnetic fields on the quiet Sun. To avoid this problem, a single noise filter with fixed cutoff corresponding to 0.5 was selected. Such a cutoff is typically obtained from the calculations of the optimum filter. The filter is equal to one until the cutoff from whereon it falls off smoothly to zero (see Fig. 3.5 a).

To reduce noise further the narrow-band images were smoothed with a $5 \times 5$ pixels window. Figure $3.5 \mathrm{~b}$ shows azimuthal averages of the power spectra from the broad-band image, from a narrow-band image after the filter is applied, and after the smoothing. They are compared with the narrow-band power spectrum when it is smeared with a "seeing-like" filter (dashed curve in Fig. 3.5 a). The latter suppresses more power than is contained in the data used here for further analysis (solid power spectrum). 


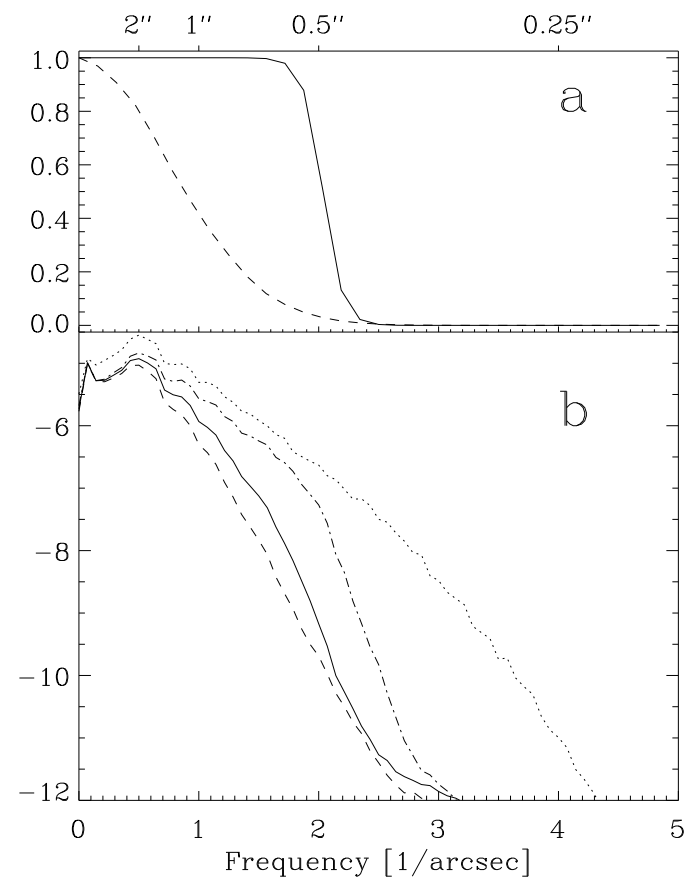

Figure 3.5: Filters and power spectra from the process of image restoration. a) Solid line: Noise filter applied to narrow-band images . Dashed line: Filter emulating a seeing with a FWHM of 0.5. b) Dotted: power spectrum of the broad-band image. Dash-dotted line: power spectrum of a narrow-band image processed with the noise filter. Solid line: power spectrum of the narrow-band image after smoothing. Dashed line: power spectrum of narrow-band image processed with the "seeing" filter. Frequencies are given in 1 /arcsec, whose inverse is also shown on the upper bound of the plots.

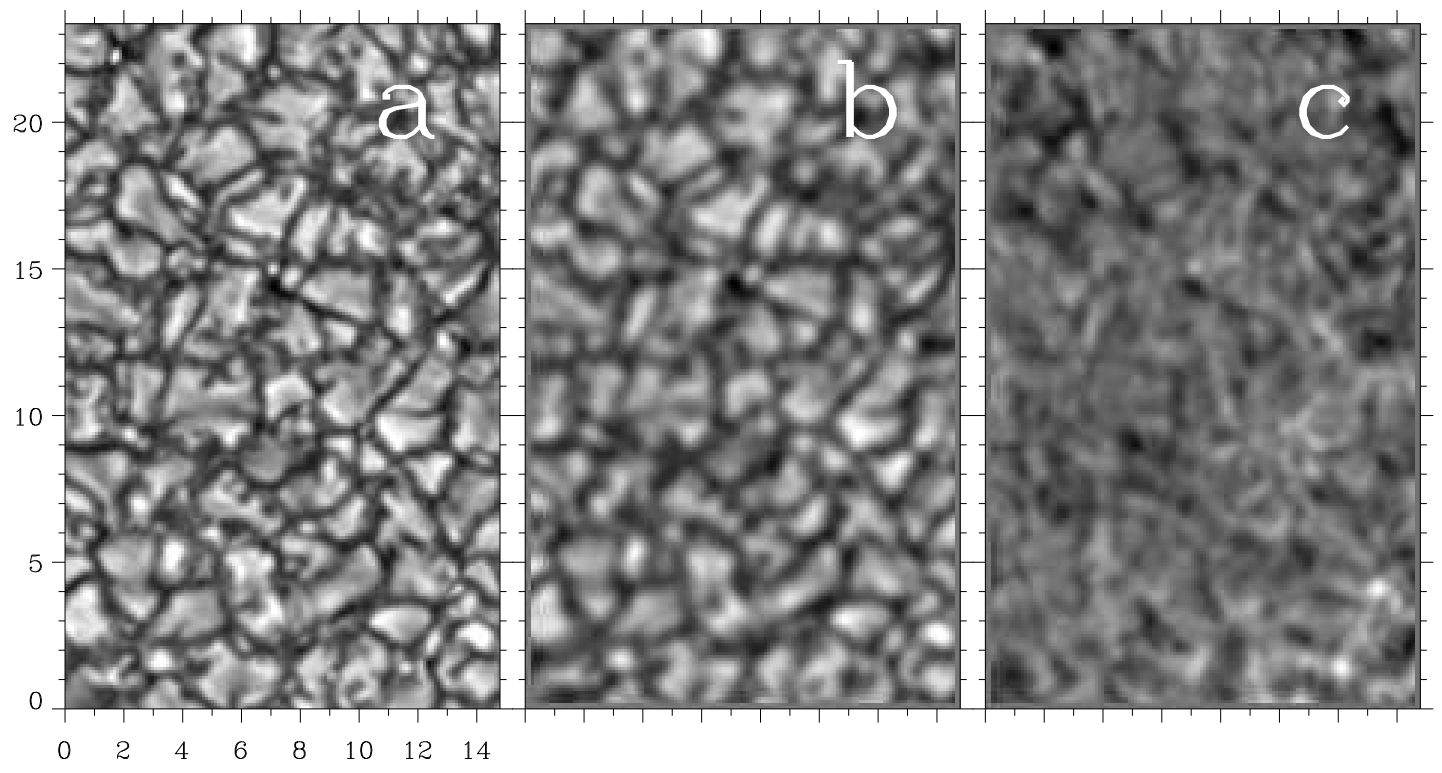

Figure 3.6: Reconstructed data. a) Broad-band image (spatial resolution $\sim 0$ '.25). b) Narrow-band continuum image (spatial resolution $\sim 00^{\prime \prime} 5$ ). c) Narrow-band image at core of Fe I $\lambda 6302.5 \AA$. The axes are in arcsec. 
Figure 3.6 shows images used in this work with a FOV of $14^{\prime \prime} \times 23^{\prime \prime}$. The original FOV is reduced due to the separation of the left and right circularly polarized images on CCD 2, and due to all the processing during the restoration. The broad-band image has a resolution of $0^{\prime \prime} .25$ with a contrast of $12 \%^{3}$, while the narrow-band continuum image has a contrast of $7 \%$. A line-core image is given in Fig. 3.6c, showing no sign of magnetic activity and thus providing another evidence that the observation was done in a very quiet Sun region.

After all this restoration the data obtained from CCD 2 is a pair of 33 images scanning the spectral range (shown in Fig. 3.4). The upper half of the CCD is scanning right circular polarization $(I+V)$ and the lower half scans the left circular polarization $(I-V)$. Adding and subtracting these images result in 2-dimensional spectra of Stokes $I$ and Stokes $V$.

\subsubsection{Crosstalk}

Usually one of the Stokes parameters measured with an instrument may be contaminated by the others. Such an effect is mainly produced by oblique reflections of the light on the mirrors in the telescope or in the instrument, becoming one of the most important problems in spectro-polarimetry. This is commonly called crosstalk.

The following experiment was performed in order to estimate the crosstalk produced by the FPI. The Stokes $V$ observed is

$$
V_{\text {obs }}=m_{I} I+m_{Q} Q+m_{U} U+m_{V} V,
$$

where $I, Q, U$ and $V$ are the Stokes parameters at the entrance of the instrument, and $m_{X}$ are the crosstalk coefficients. These constants are the fraction of contamination from Stokes $X$ to Stokes $V$ induced by the FPI. The ideal case is $m_{V}=1$ and $m_{I}=m_{Q}=m_{U}=0$. In general the latter three are not zero, and they must be estimated. The insertion of a linear polarizer in front of the FPI (on the left side of the scheme in Fig. 3.2) produces a vector of Stokes parameters like this

$$
\left(\begin{array}{l}
I \\
Q \\
U \\
V
\end{array}\right)_{L P}=I\left(\begin{array}{c}
1 \\
\cos 2 \theta \\
\sin 2 \theta \\
0
\end{array}\right)
$$

with $\theta$ the angle of the linear polarizer with regard to the vertical direction. The observed Stokes $V$ from Eq. 3.11 results in

$$
V_{o b s}=I\left(m_{I}+\cos 2 \theta m_{Q}+\sin 2 \theta m_{U}+0 m_{V}\right) .
$$

\footnotetext{
${ }^{3}$ With the definition of contrast as the standard deviation of intensity of the image divided by the average value of intensities.
} 
Measuring $V_{o b s}$ with three different angles $\left(0^{\circ}, 45^{\circ}\right.$ and $\left.90^{\circ}\right)$ and considering $I$ as the intensity observed, yields $m_{I}=0.016, m_{Q}=0.008$ and $m_{U}=0.027$. Stokes $Q$ and $U$ possess similar level of polarization as Stokes $V^{4}$. Since $m_{Q}$ and $m_{U}$ are only some few percent this crosstalk can be neglected. However, crosstalk from Stokes $I$ may not be ignored, due to the small polarization signals expected from IN. It can be corrected when the left and right circular polarization profiles $(I+V$ and $I-V)$ are normalized to the continuum before subtracting them. In general the quantities measured in the two parts of CCD 2 are

$$
\begin{aligned}
& S_{1}=f_{1}(I+V) / 2 \\
& S_{2}=f_{2}(I-V) / 2,
\end{aligned}
$$

thus Stokes $I$ and Stokes $V$ are

$$
\begin{aligned}
& I=S_{1}+S_{2} \\
& V=S_{1}-S_{2},
\end{aligned}
$$

After the normalization the factors are $f_{1}=f_{2}=1$, and $V$ is free of crosstalk from $I$.

\subsubsection{Magnetograms}

A fast way to obtain a magnetogram from Stokes $V$ maps is using the magnetograph equation (see Landi Degl'Innocenti 1992). This equation takes Stokes $V$ proportional to the longitudinal component of the magnetic field in the weak field approximation

$$
V(\lambda)=C(\lambda) B,
$$

where $C(\lambda)$ contains the derivative of Stokes $I$ with respect to the wavelength, the Landé factor $g_{L}$, and the square of the central wavelength of the line $\left(\lambda_{0}\right)$ :

$$
C(\lambda)=k g_{L} \lambda_{0}^{2} \frac{d I(\lambda)}{d \lambda},
$$

with $k$ a constant, $k=-4.67 \times 10^{-13}\left[\AA^{-1} \mathrm{G}^{-1}\right]$. The calibration, i.e. the determination of $C(\lambda)$, is done for each individual pixel in the FOV and for each line separately. In classical magnetographs only a measurement in one wing of the line is used for calibration (like the magnetogram of Fig. 1.1). Instead of using this traditional method, more than one measurement (i.e. more than one point in the Stokes $V$ profile) is considered to make a least-squares fit to Eq. 3.16 to obtain the magnetic field

$$
B_{\mathrm{eff}}=\frac{\sum_{j} V\left(\lambda_{j}\right) C\left(\lambda_{j}\right)}{\sum_{j} C^{2}\left(\lambda_{j}\right)} .
$$

\footnotetext{
${ }^{4}$ In the quiet Sun they are generally smaller.
} 
The wavelengths $\lambda_{j}$ taken for the summations are the two extrema of the derivative of Stokes $I$ and their adjacent points on the profiles. The statistical use of these six wavelength positions reduces the noise in the determination of the magnetic field. $B_{\text {eff }}$ is only an estimation because, in general, Stokes $I$ and Stokes $V$ do not satisfy Eq. 3.16 derived from the weak field approximation. Moreover, it can only give an estimation on the magnetic flux density averaged over the resolution element, and never on the magnetic field strength, as the magnetic structures under study are very likely much smaller than the resolution element. In this case

$$
B_{\text {eff }} \simeq \alpha B,
$$

with $\alpha$ the area filling factor of the magnetic structure with a magnetic field $B$, in a remaining non magnetic surface.

One source of error in these determinations is the random noise of the Stokes $V$ spectra. A study on error propagation for Eq. 3.18 may give an idea on the influence of this noise:

$$
\Delta B_{\mathrm{eff}}^{2} \simeq \sum_{j}\left[\partial B_{\mathrm{eff}} / \partial V\left(\lambda_{j}\right)\right]^{2} \Delta V\left(\lambda_{j}\right)^{2} \simeq \Delta V^{2} / \sum_{j} C^{2}\left(\lambda_{j}\right)
$$

with $\Delta V$ the rms noise of Stokes $V$. This rms is usually obtained from the continuum near the line profile. However, the data in this Chapter have almost no continuum points, most wavelength positions contain Stokes $V$ signals and there are no positions far from a line. Hence, $\Delta V$ was inferred from the representation of the standard deviation of $V\left(\Delta V_{j}\right.$ at each individual pixel $)$ versus $B_{\text {eff. }}$ The average of $\Delta V_{j}$ for magnetic flux extrapolated to zero is a good estimation of the noise of Stokes $V$. The value obtained was $5 \times 10^{-3} I_{c}$, with $I_{c}$ the continuum intensity. Using this value in Eq. 3.20 the figures for the noise in the magnetic field are

$$
\begin{aligned}
& \Delta B_{\text {eff }} \simeq 23 \mathrm{G} \text { for Fe I } \lambda 6301.5 \AA, \\
& \Delta B_{\text {eff }} \simeq 17 \text { G for Fe I } \lambda 6302.5 \AA .
\end{aligned}
$$

These are average values of the noise over the FOV. Each individual pixels has its own value, depending mainly on the Stokes $I$ profiles (pixel in intergranular lanes have slightly larger uncertainties)

A second source of error is the crosstalk from intensity to circular polarization. Due to the low number of wavelengths scanned through the spectral lines there are almost no continuum points (see Fig. 3.4). In this case, the normalization of $S_{1}$ and $S_{2}$ in Eq. 3.14 is done for only two points in the spectra of Stokes $I$. Thus it is affected by noise resulting that $f_{1}$ and $f_{2}$ are different from 1 and this 
crosstalk is not totally removed. Instead of the true circular polarization the difference yields

$$
\widetilde{V}=S_{1}-S_{2}
$$

where $\widetilde{V}$ can be contaminated by the intensity:

$$
\widetilde{V} \simeq V+m_{I \rightarrow V} I
$$

where $m_{I \rightarrow V}=\frac{f_{1}-f_{2}}{2}$ is the remaining crosstalk and $V$ is the true circular polarization. This crosstalk produced by noise must be of the order of the $\Delta V$ estimated before. Since $I$ is much larger than $V$ (two orders of magnitude for IN signals) this crosstalk can become a serious problem. From Eq. 3.23 the magnetic flux without crosstalk can be expressed as

$$
B_{\text {eff } 0}=B_{\text {eff }}-m_{I \rightarrow V} \Delta B
$$

where $B_{\text {eff }}$ in the flux calculated from $\widetilde{V}$ and $\Delta B$ the contribution from the intensity $I$. This contribution is the "magnetic flux" calculated for $V=I$ meaning that Stokes $V$ must be replaced by Stokes $I$ in Eq. 3.18:

$$
\Delta B=\frac{\sum_{j} I\left(\lambda_{j}\right) C\left(\lambda_{j}\right)}{\sum_{j} C^{2}\left(\lambda_{j}\right)} .
$$

This estimation for Stokes $I$ profiles from quiet Sun gives $\Delta B \sim 150$ G. Since the crosstalk $m_{I \rightarrow V}=\frac{f_{1}-f_{2}}{2}$ is smaller than a few per cent the contribution of the intensity is negligible (only a few $\mathrm{G}$ ) compared to the measured values (see Sect. 3.4.1). The method using both lobes of Stokes $V$ to obtain the flux has thus become an efficient technique to avoid crosstalk from $I$ to $V$. The use of only one wing of the line to determine the magnetic field leads to $\Delta B \sim 3 \times 10^{3}$ $\mathrm{G}$ resulting in a huge systematic error in the determination of the flux. The difference with respect to the two-wings procedure is due to the cancellation of positive and negative contributions in Eq. 3.25. 


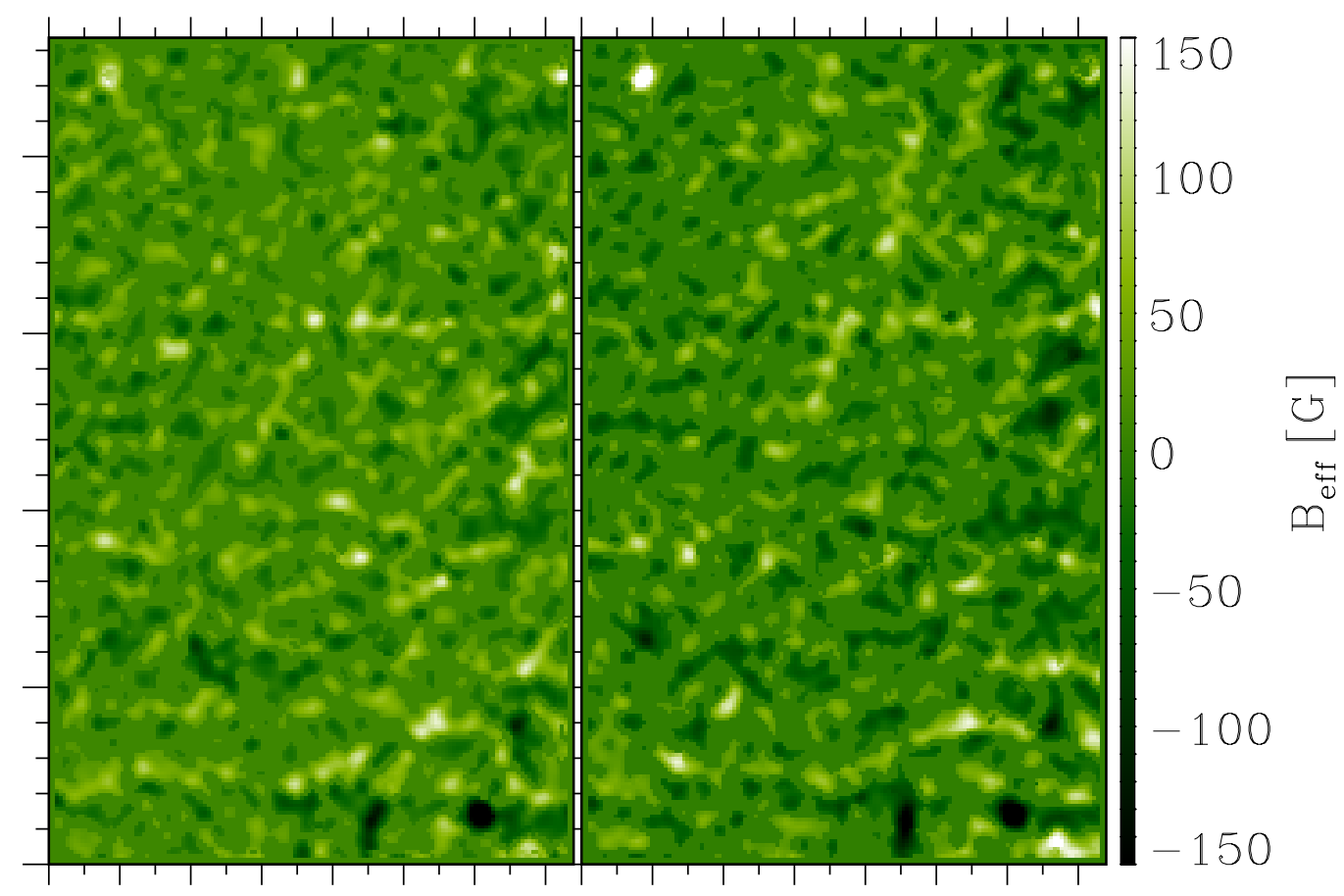

Figure 3.7: Magnetograms for both iron lines, Fe I $\lambda 6302.5 \AA$ (left) and Fe I $\lambda 6301.5 \AA$ (right). Signals below noise level are set to zero, and bright or dark distinguish between polarities. Distance between ticks is $1^{\prime \prime}$.

\subsection{Results}

\subsubsection{Flux density}

Figure 3.7 shows magnetograms obtained in the way descrived above. Pixels with magnetic field below noise level are set to zero. About $45 \%$ of the FOV is filled with magnetic fields, for both lines. Both magnetograms from Fe I $\lambda 6302.5 \AA$ (left) and Fe I $\lambda 6301.5 \AA$ (right) exhibit a salt and pepper pattern with a similar distribution of patches. This observation with limited magnetic sensitivity shows the largest amount of magnetic flux ever observed in the IN thanks mainly to the improvement of the spatial resolution (see Sect. 3.4.7). 
This unsigned magnetic flux is defined as

$$
\overline{\left|B_{\mathrm{eff}}\right|} \equiv \frac{1}{N} \sum_{j}\left|B_{\mathrm{eff}}^{j}\right|,
$$

with $\mathrm{N}$ the total number of pixels in the FOV and $\left|B_{\text {eff }}^{j}\right|$ the absolute value of the magnetic field in pixel $j$, once signals below noise are set to zero. The values obtained for the unsigned flux are

$$
\overline{\left|B_{\text {eff }}\right|}=\left\{\begin{array}{l}
21 \mathrm{G}, \text { for Fe I } \lambda 6301.5 \AA \\
17 \mathrm{G}, \text { for Fe I } \lambda 6302.5 \AA
\end{array}\right.
$$

A method to evaluate the effect of the noise associated with each individual magnetic field $\left(\Delta B_{j}\right)$, is to carry out a Monte-Carlo simulation (see Press et al. 1992). From a sample of $N$ values (from $N$ pixels), it is possible to create $M$ different samples just adding random noise $M$ times to each value with a standard deviation equal to the individual noise of each value. This noise must be as random as possible to avoid tendencies in the results. The results calculated for each sample give $M$ solutions. The average of these solutions is the final result, and the standard deviation is the noise of this result.

To avoid the influence of the noise in Eq. 3.26, the random noise must be added after calculating the absolute value of the magnetic field. In this case the $M$ samples are

$$
\left\{\left|B_{\text {eff }}^{\prime}\right|^{j, i}\right\}_{j=1, \ldots, N}=\left\{\left|B_{\text {eff }}^{j}\right|+R\left(\Delta B_{j}\right)\right\}_{j=1, \ldots, N}, \quad(i=1, \ldots, M)
$$

where $R\left(\Delta B_{j}\right)$ is the random noise of the $j$ values $\left(\Delta B_{j}\right),\left|B_{\text {eff }}^{\prime}\right|^{j, i}$ are the $j$ new values for sample $i$, and $B_{\text {eff }}^{j}$ are the $j$ values of the "mother" sample.

The above calculation shows the robustness of the estimates (3.27) since the resulting noise is negligible, pointing out that the average of absolute values and the use of a threshold do not affect the result.

The signed magnetic flux can be obtained keeping the sign of the magnetic field in Eq. 3.26, instead of taking the absolute value (i.e. averaging $B_{\text {eff }}^{j}$ ). Such calculation yields

$$
\overline{B_{\text {eff }}}=\left\{\begin{array}{l}
+2 \mathrm{G}, \text { for Fe I } \lambda 6301.5 \AA \\
+3 \mathrm{G}, \text { for Fe I } \lambda 6302.5 \AA
\end{array}\right.
$$

A similar low level of imbalanced flux has already been observed earlier with these lines (Lites 2002). 


\subsubsection{Magnetic field strength}

The ratio method can be used to estimate the magnetic field strength using the magnetic flux computed above. This method was used for the first time by Stenflo (1973) to determine the intrinsic fields of the unresolved magnetic structures of the network. The technique is based on the comparison of the flux measured with two different lines.

The magnetograms obtained from both lines are well correlated (see Fig. 3.7), but the effective flux estimated with Fe I $\lambda 6301.5 \AA$ is larger than the flux obtained from Fe I $\lambda 6302.5 \AA$. This difference must be obtained from all magnetic signals within the FOV, since noise prevents any conclusion from individual measurements. Using a least-squares fit the ratio of the fluxes from the two lines is

$$
\frac{B_{\text {eff }}(6301)}{B_{\text {eff }}(6302)} \simeq 1.25 \pm 0.14
$$

This ratio is clearly above 1 , and this is only possible when the lines are not formed under conditions of the weak field aproximation and have started to saturate magnetically. In the weak field regime, Stokes $V$ grows linearly with the magnetic field strength but when the Zeeman splitting starts to dominate over the broadening of the line the level of polarization does not increase any more (see Section 2.2 and Fig. 2.3).

The magnetic field strength needed for this saturation of Stokes $V$ to happen in a spectral line depends on the broadening of the line and on its Landé factor. Since both lines are very similar and have the same broadening the only parameter to take into account is $g_{L}$. As Fe I $\lambda 6301.5 \AA$ is less sensitive to magnetic fields than Fe I $\lambda 6302.5 \AA$, it needs stronger fields to saturate, thus the flux measured from Fe I $\lambda 6301.5 \AA$ can only be larger than for Fe I $\lambda 6302.5 \AA$ $\left(B_{\text {eff }}(6301) / B_{\text {eff }}(6302)>1\right)$, when the weak field approximation does not apply.

Figure 3.8 shows how this can happen. Synthetic profiles of both lines are compared with the derivative used to determine the magnetic flux as presented in Section 3.4.1. The comparison is done for a field of $150 \mathrm{G}$ (top) where the weak field approximation fits perfectly and for $1500 \mathrm{G}$ (bottom) where it fits better for Fe I $\lambda 6301.5 \AA$ than for Fe I $\lambda 6302.5 \AA$. The result is that the first line measures more flux than the second one.

It is possible to calibrate this ratio to have an estimation of the real magnetic field strength. Figure 3.9 shows synthetic ratios computed for different magnetic field strengths. Three different model atmospheres have been taken into account. The first considers an atmosphere with the same properties as the quiet Sun. 

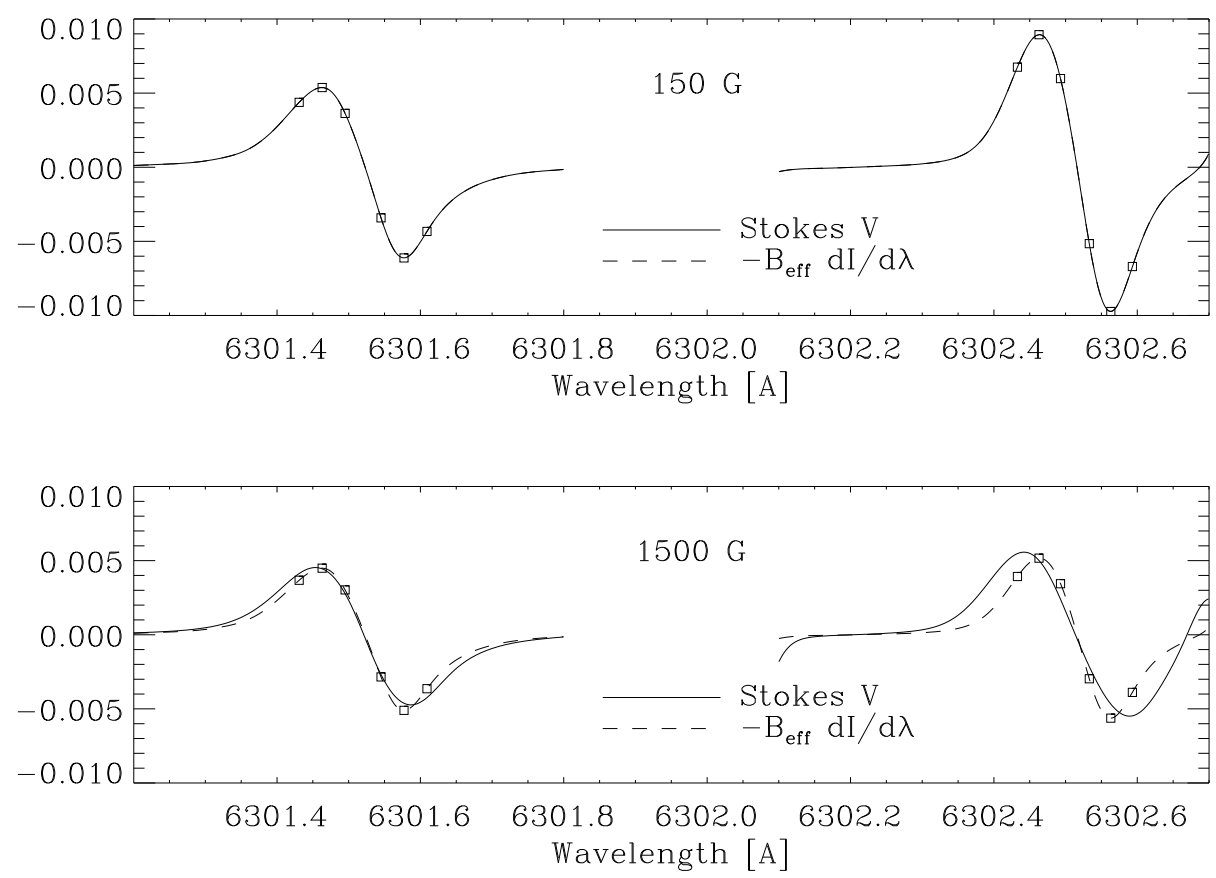

Figure 3.8: Comparison of synthetic Stokes $V$ profiles (solid) with derivatives of Stokes $I$ (dashed) for weak and strong magnetic fields. Squares indicate the wavelength positions used in the determination of $\mathrm{B}_{\text {eff }}$.

Under the assumption of a longitudinal magnetic field the synthetic profiles can be computed as

$$
\begin{aligned}
& V_{m}(\lambda)=\frac{1}{2}\left[I_{q}\left(\lambda-\Delta \lambda_{B}\right)-I_{q}\left(\lambda+\Delta \lambda_{B}\right)\right], \\
& I_{m}(\lambda)=\frac{1}{2}\left[I_{q}\left(\lambda-\Delta \lambda_{B}\right)+I_{q}\left(\lambda+\Delta \lambda_{B}\right)\right],
\end{aligned}
$$

where $I_{q}$ is Stokes $I$ produced by an unmagnetized quiet Sun and $\Delta \lambda_{B}$ is the Zeeman splitting (see Eq. 2.8). In case the resolution element is filled only with a fraction $\alpha$ by magnetic fields the resulting profiles are

$$
\begin{aligned}
V(\lambda) & =\alpha V_{m}(\lambda), \\
I(\lambda) & =\alpha I_{m}+(1-\alpha) I_{q}(\lambda) .
\end{aligned}
$$

The study was done changing the field strength, but preserving a constant flux ( $\alpha B=30 \mathrm{G}$, a typical value in the magnetograms of Fig 3.7). This method was used to compute the Stokes $V$ profiles shown in Fig. 3.8. The ratio obtained for weak fields is equal to 1 (see blue curve in Fig. 3.9), while it increases up to 1.4 for field strength of $1.5 \mathrm{kG}$. The observed ratio (Eq. 3.30 and horizontal line in Fig. 3.9) requires a magnetic field of $\sim 1.2 \mathrm{kG}$. 


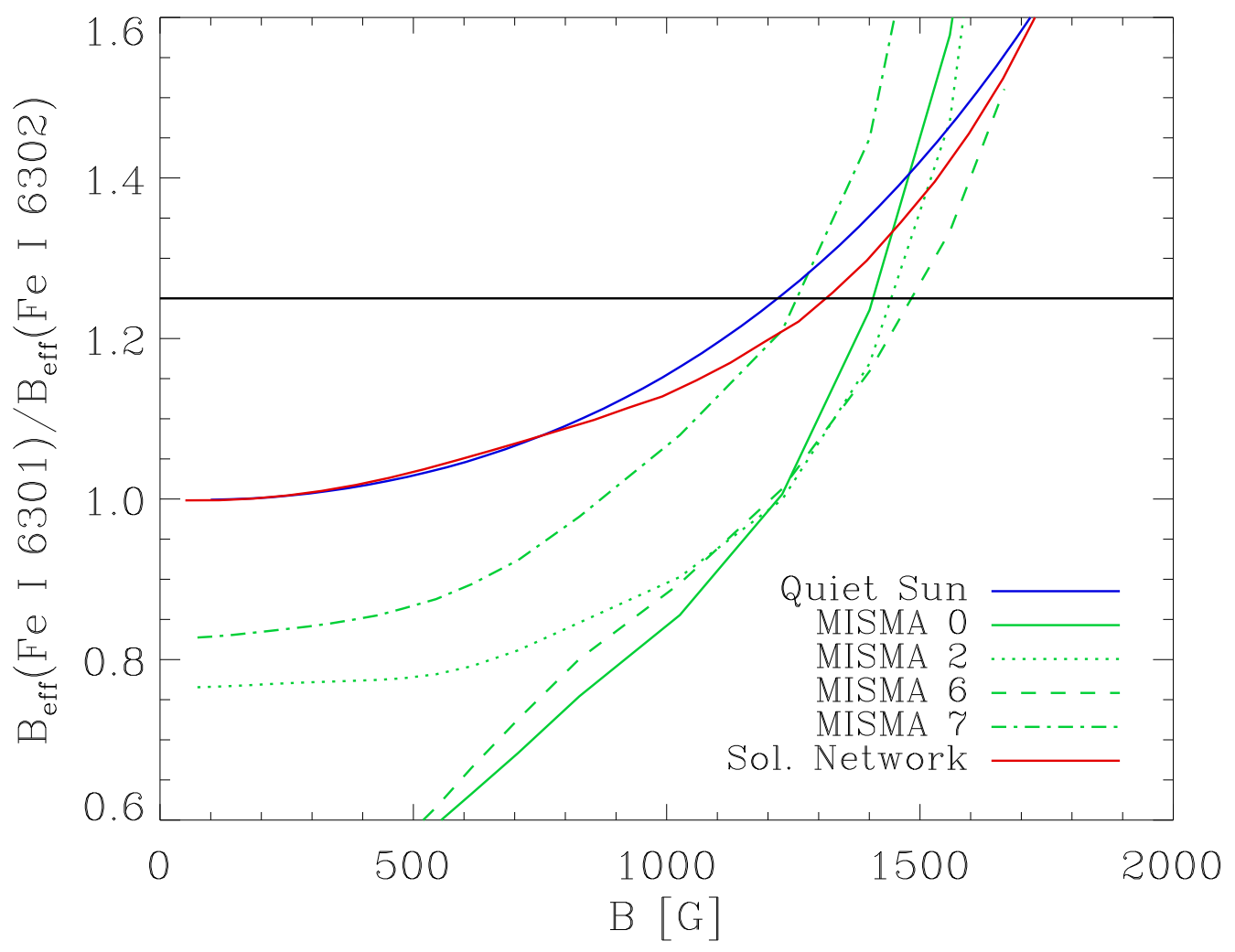

Figure 3.9: Ratio of the effective magnetic fields between Fe I $\lambda 6301.5 \AA$ and Fe I $\lambda 6302.5 \AA$ versus the magnetic field strength for different models of the solar atmosphere (in different colors). Observed ratio (horizontal line) agrees with models for intrinsic fields larger than $1 \mathrm{kG}$.

The second model used is a hot model atmosphere (Solanki 1986, network with constant magnetic field, constant microturbulence of $1 \mathrm{~km} \mathrm{~s}^{-1}$, and a macroturbulence of $1 \mathrm{~km} \mathrm{~s}^{-1}$ ). The variation of the ratio with field strength (red curve in Fig. 3.9) is very similar to that for the quiet Sun.

Finally, a third type of model is used for the calibration, the MISMA hypothesis by Sánchez Almeida \& Lites 2000 (see Section 4.6). This atmosphere can reproduce quiet Sun profiles observed at disk center, including their asymmetries. The magnetic field strength of the model MISMAs varies with height in the atmosphere. The field strength of the original models is modified by changing the magnetic field strength at the base of the atmosphere, and then recomputing the full vertical stratification. The mean magnetic field of the 
atmosphere represented in Fig. 3.9 is chosen as the magnetic field strength at the height where the line core opacity of Fe I $\lambda 6302.5 \AA$ equals one. The variation of the synthetic ratio versus the mean magnetic field strength is qualitatively different from the variation found in the two previous cases. However, the difference is significant only when the magnetic field is weak. The observed ratio (Eq. 3.30) also requires $\mathrm{kG}$ magnetic field strengths for MISMAs. The reason why the ratio $B_{\text {eff }}(6301) / B_{\text {eff }}(6302)$ does not tend to one for weak fields has to do with the stray-light contamination of the model MISMAs (a contribution equivalent to the term $[1-\alpha] I_{q}$ in Eq. 3.32). It has a velocity distribution very different from the velocities of the magnetized plasma. Then the magnetized and un-magnetized atmospheres are no longer similar, which causes the breakdown of the magnetograph equation (Eq. 3.16). Figure 3.9 includes several kinds of MISMAs, from simple ones (classes 0 and 2) to atmospheres having opposite polarities in the resolution element (classes 6 and 7). All of them assign the same magnetic field strength to the observed ratio (Eq. 3.30).

Structures with intrinsic kG magnetic field strength showing $20 \mathrm{G}$ flux density have to occupy only a small fraction of the solar surface. The simple twocomponent model allows to estimate the area coverage or filling factor. The filling factor is just the ratio between effective and intrinsic field strengths (Eq. (3.19)). If $B \sim 1 \mathrm{kG}$ then

$$
\alpha \sim \frac{B_{\text {eff }}}{B} \sim \frac{20 \mathrm{G}}{1000 \mathrm{G}} \sim 0.02
$$

Only $2 \%$ of the solar surface produce the observed signal. We observe them to cover as much as $45 \%$ of the FOV because of the limited spatial resolution of the observations. One can also employ the same order-of-magnitude calculation to estimate the size $l$ of the magnetic concentrations. If $M$ magnetic concentrations occupy a resolution element of size $L$, then

$$
\alpha \sim\left(\frac{l}{L}\right)^{2} M
$$

which, together with Eq. (3.19), renders

$$
l \sim L \sqrt{\frac{B_{\text {eff }}}{M B}} \sim\left\{\begin{array}{c}
75 \mathrm{~km} \text { for } M=1 \\
25 \mathrm{~km} \text { for } M=10 \\
7 \mathrm{~km} \text { for } M=100
\end{array}\right.
$$

We use $L=363 \mathrm{~km} \widehat{=} 0^{\prime \prime} .5, B=1 \mathrm{kG}$, and $B_{\text {eff }}=40 \mathrm{G}$, the latter being the typical value of the signals above noise in the magnetograms (Fig. 3.7). 


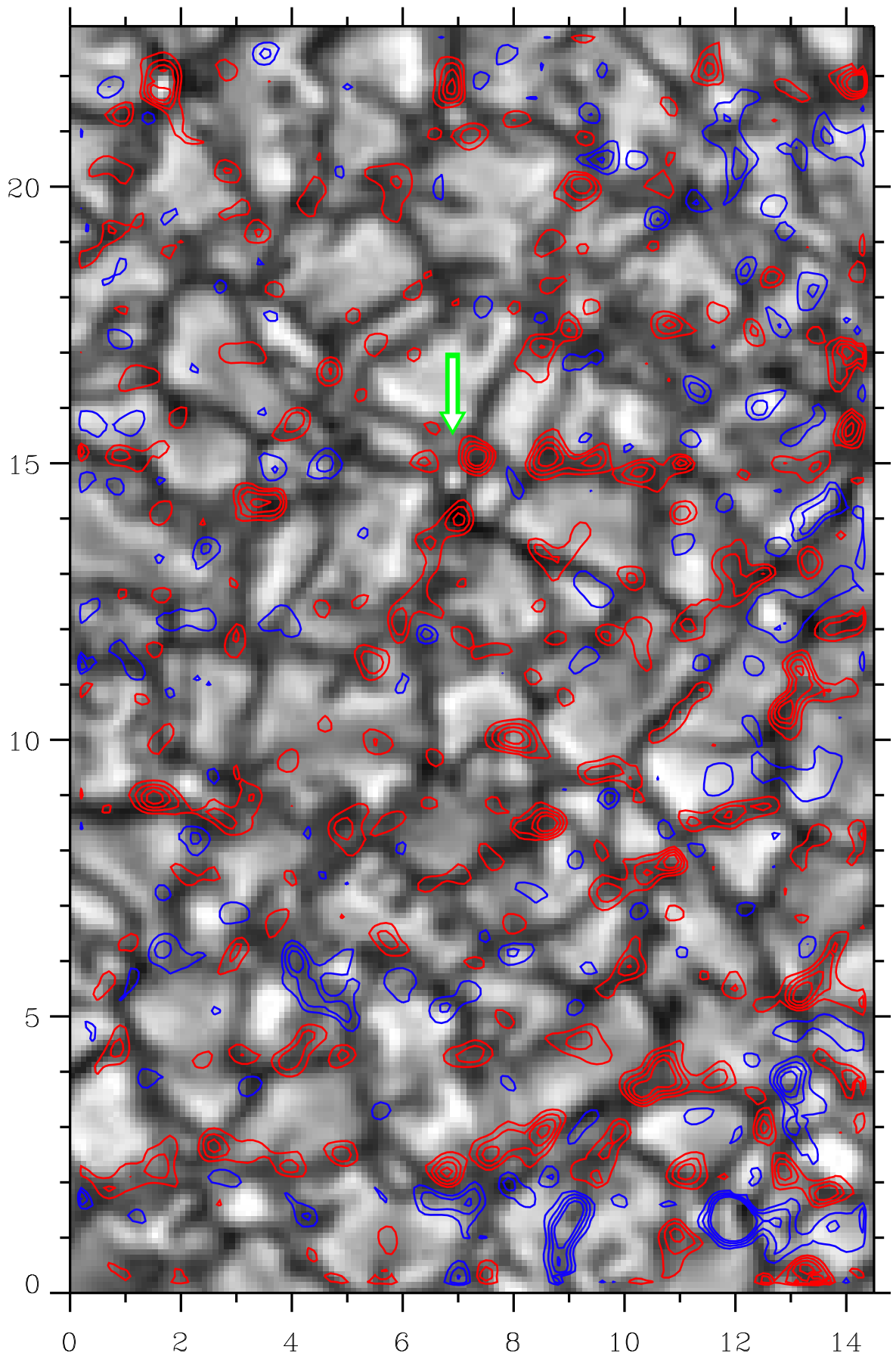

Figure 3.10: Magnetogram for Fe I $\lambda 6302.5 \AA$ overlaid over the speckle reconstructed broad-band image. Contours belong to $\left|B_{\text {eff }}\right|=30$, 50, 70 and $90 \mathrm{G}$. Red and blue contours distinguish between the two polarities. Axes in arcsec. 


\subsubsection{Spatial distribution}

Figure 3.10 shows the magnetogram obtained from Fe I $\lambda 6302.5 \AA$ data overlaid on the broad-band reconstructed image. Red and blue contours separate the two polarities and correspond to effective magnetic fields of 30, 50, 70 and $90 \mathrm{G}$. At first sight, it is clear that most of the magnetic fields are located in the intergranular lanes. Such tendency was observed before (Lin \& Rimmele 1999; Lites 2002; Khomenko 2003) and was predicted by MHD simulations of magnetoconvection (e.g., Cattaneo 1999; Vögler \& Schüssler 2003).

A more quantitative evidence of the preference of the magnetic fields to stay at intergranules is depicted in Fig. 3.11. It shows histograms for the number of pixels versus continuum intensity and versus the velocity. There is a big difference depending on whether the pixels contain magnetic signals above noise (solid lines)
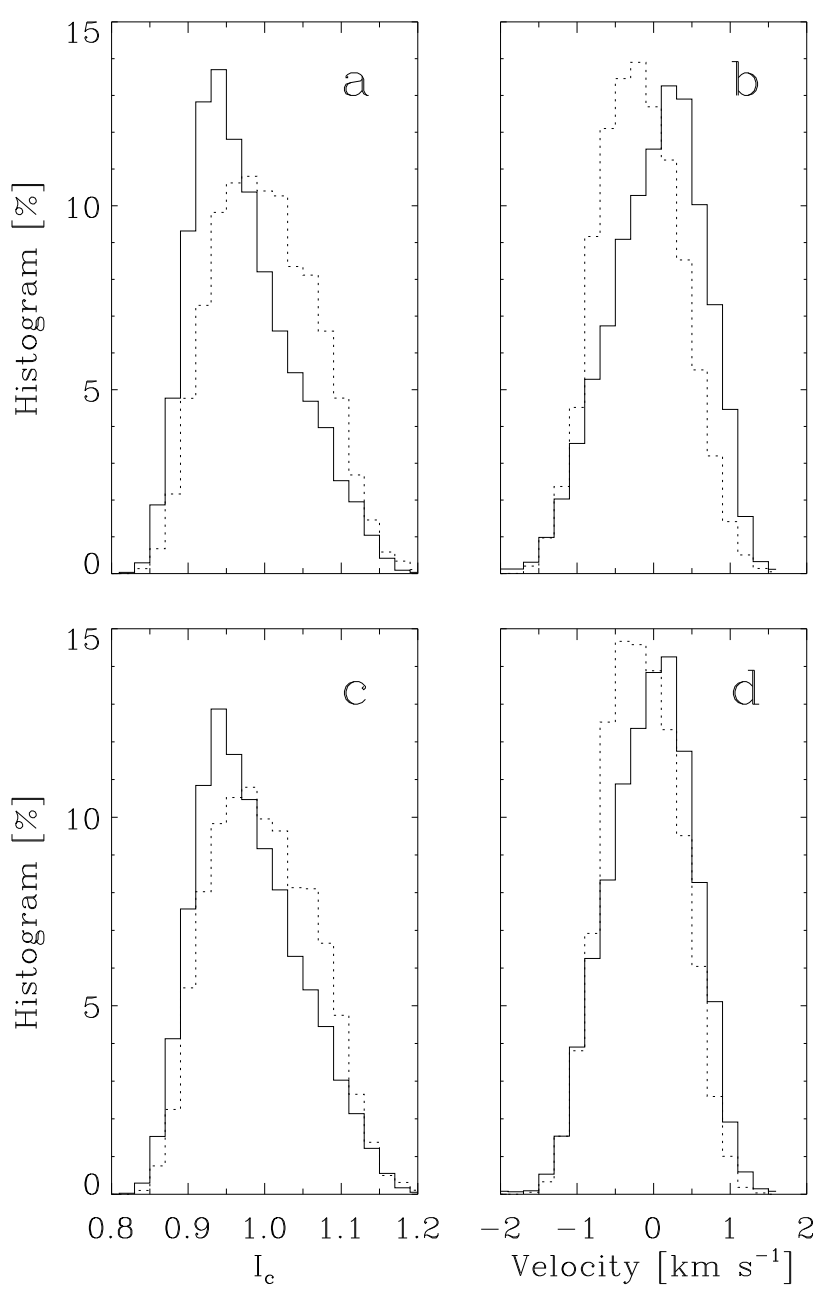

Figure 3.11: Histograms for continuum intensities (a and $\mathbf{c}$ ) and velocities (b and $\mathbf{d})$ for pixels with magnetic flux above noise (solid lines) and below noise level (dotted lines). Upper panels represent results from Fe I $\lambda 6302.5 \AA$ and lower panels from Fe I $\lambda 6301.5 \AA$. Intensities are given relative to their average over the FOV and the average velocity is defined zero. Redshifts are positive. 
or not (dotted lines). About $65 \%$ of the magnetic signals are found in pixels with low intensity $\left(I_{c}<1\right)$ and $60 \%$ of these pixels exhibit redshifts. These findings are both indications that magnetic fields are concentrated in the dark intergranular lanes associated with downflows. This phenomenon can be explained with the concept of frozen-in magnetic fields. In the case of small magnetic structures the granular motion dominates over the magnetic field so the plasma flow carries the flux tubes to the intergranules.

Another peculiarity from this study has to do with the imbalance of flux present in the magnetograms (Eq. 3.29). Without expanding on this point with detailed descriptions, note that the intergranular lanes seem to carry almost all of this imbalanced flux, while the granules themselves show balanced magnetic flux.

The magnetic structures found here harbour $\mathrm{kG}$ fields. This implies that they should be bright features in the broad-band images on the following ground. A flux tube with $\mathrm{kG}$ fields has a strong magnetic pressure producing a reduction of density of the magnetized plasma, decreasing also the opacity with respect to the surroundings. Thus the light in the flux tube comes from deeper layers, where the plasma is hotter and produces more photons than the ambient non-magnetic area (the so called hot-wall effect; Spruit 1976). However in Fig. 3.10 there are no bright points associated with magnetic fields, with some exceptions at position $\left[2^{\prime \prime}, 22^{\prime \prime}\right]$, or $\left[12^{\prime \prime}, 1^{\prime \prime}\right]$. This can be due to the still not sufficient resolution to see such brightness. Another explanation can be that our preliminary picture of magnetic fields in the quiet Sun is misleading and brightenings must not be expected.

The FOV contains a particular structure. There is a bright point surrounded by magnetic fields (indicated by the arrow in Fig. 3.10), but no magnetic signal appears just on its position. This exceptional structure can be explained when there is no magnentic field associated to the bright point. However the presence of magnetic field around it and the rareness of a such structures, suggests an alternative solution to the absence of polarization. Sánchez Almeida (2000) pointed out the possibility to find an evacuated flux tube with not enough plasma to produce polarized light. Thus Stokes $V$ profiles with only a very small level of polarization. Since the magnetic feature is almost empty of plasma the light comes from deep layers and the structure appears as a bright point. 


\subsubsection{Temporal evolution}

The short duration of the time series taken (20 snapshots during 17 minutes, see Section 3.2) and the variable quality of the series make it difficult to study the temporal evolution of the IN magnetic fields in depth. However some conclusions can be obtained in form of qualitative aspects of the dynamics.

Figures 3.12 and 3.13 show the temporal evolution of IN fields in two different small regions from the FOV of Fig. 3.10. Elapsed time is displayed in the top right corners. The single magnetogram studied in the above sections is the one at $7^{\mathrm{m}} 30^{\mathrm{s}}$. In general, both figures show magnetic signal remaining present for some time or even during the whole series. Magnetic flux patches, located in intergranular lanes and lasting for a long time, are likely not single magnetic field structures with long lifetime. Simulations of magnetoconvection in the quiet Sun show persistent downdrafts continuously fed with magnetic fields carried by the plasma (Cattaneo 1999; Emonet \& Cattaneo 2001). This process gives the impression of a single structure, while in reality magnetic field is continuously reprocessed. The present result can serve as an important observational constraint to calculate the correct efficiency of the granulation in quiet Sun to process magnetic fields, or to compute the creation time scale of the IN flux.

A clear evidence revealing cancellation of magnetic fields of opposite polarity is shown in Fig. 3.12. The arrow points to a very stable negative magnetic patch (associated with a small bright feature) remaining during the whole series. This structure seems to be eating positive magnetic structures in the surroundings. Both, positive and negative structures, are interacting but the cancellation does not come to an end. The above explanation for the longlivety of some magnetic structure is also valid here, both patches of opposite polarity are all the time gathering magnetic fields from their surroundings with the impression of stability, when in reality the process is dynamically active and continuous field cancellation is going on. Such interactions are also visible in the simulations by Cattaneo (1999).

The arrow in Fig. 3.13 points to the same particular point as in Fig. 3.10. This particular structure shows a bright point with no magnetic signal but surrounded by magnetic features (see Section 3.4.3). The temporal evolution of the bright point shows magnetic fields associated with it just before and after the snapshot at $7^{\mathrm{m}} 30^{\mathrm{s}}$. A possible explanation of this behaviour is given by Sánchez Almeida (2000). A small increase in the magnetic field concentration can evacuate the flux tube totally resulting in an absence of magnetized gas and thus an absence of polarized radiation. Such a phenomenon may occur in small intervals of time, their observable signature may look similar as a creation-destruction process of magnetic fields when in reality its changes in polarization signals are due to evacuation of the magnetic flux tube. Thus it should be another caveat in the determination of the rates of creation-destruction of magnetic fields. 


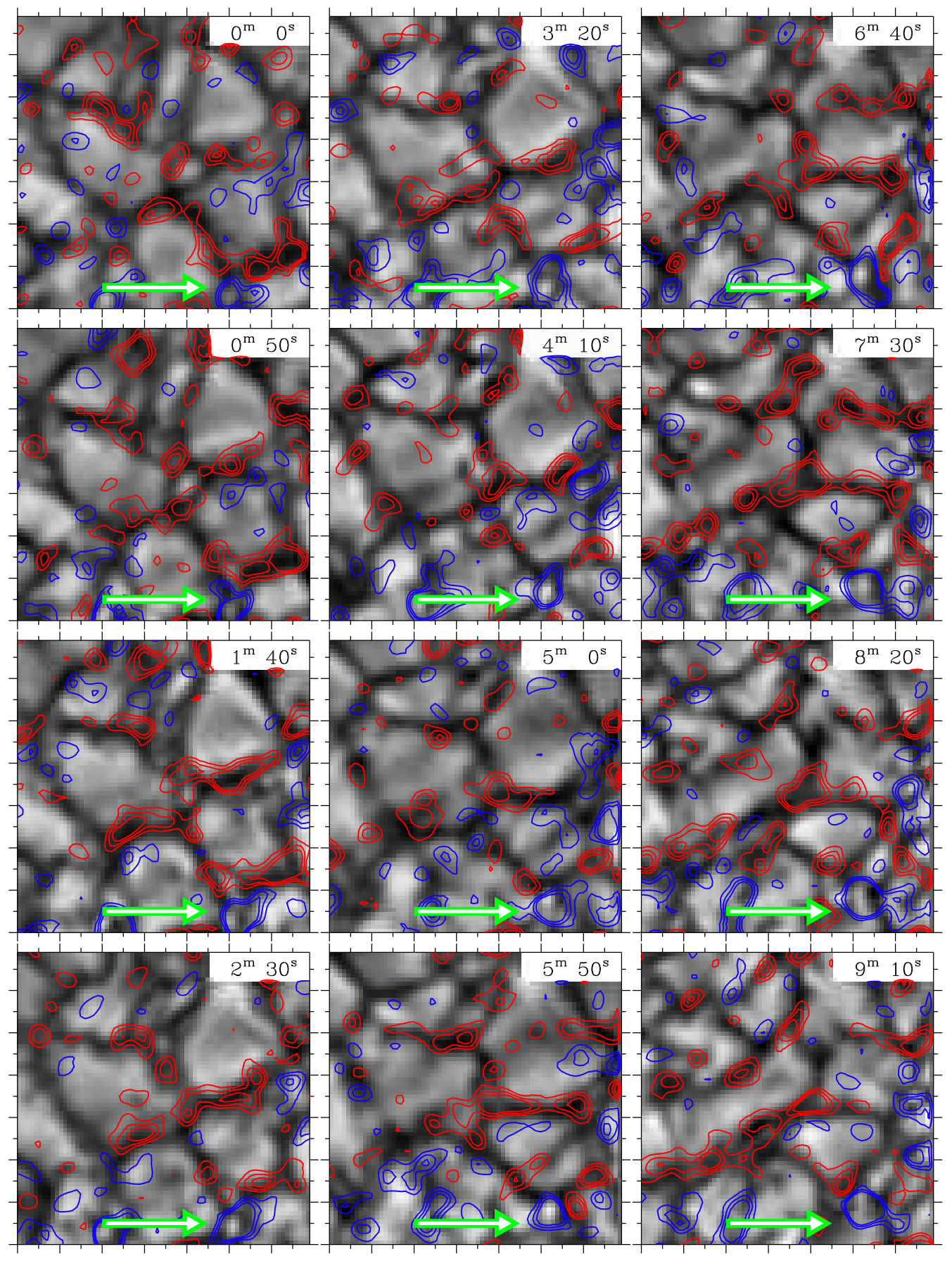

Figure 3.12: Time sequence of magnetograms overlaid onto intensity images (as in Fig. 3.10) of $7^{\prime \prime} \times 7^{\prime \prime}$ FOV, with time step of 50 sec. Arrow points to a region dominated by negative magnetic field seemingly eating positive patches. 


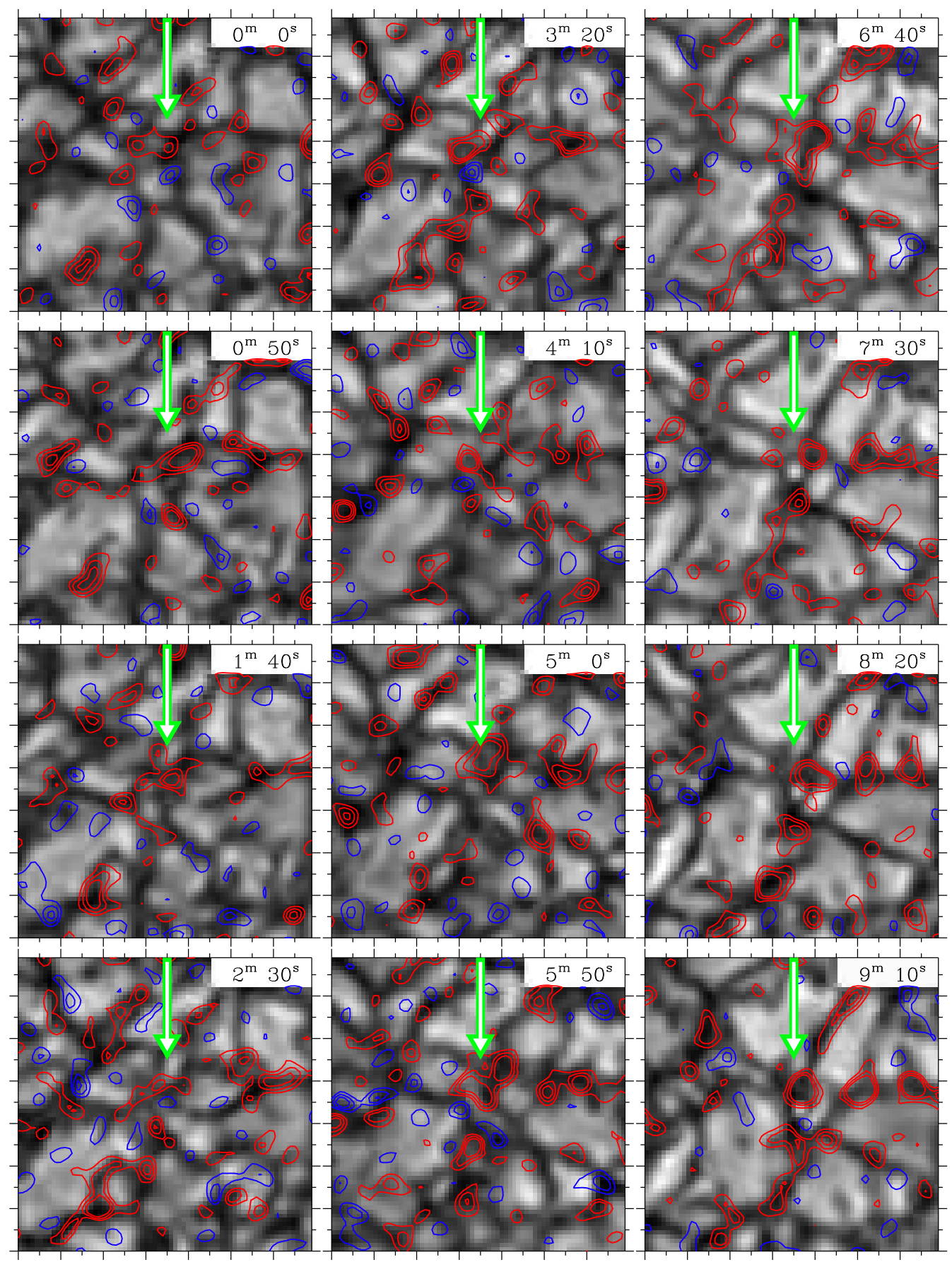

Figure 3.13: Same as Fig. 3.12 for another FOV. Arrow as in Fig. 3.10 where some cancellations occur and finally a bright point surrounded by magnetic fields appears (see text). 
Figure 3.14: Magnetogram of the FOV showing strongest signals with a threshold of $50 \mathrm{G}$. The red circle has a diameter of $5^{\prime \prime}$.

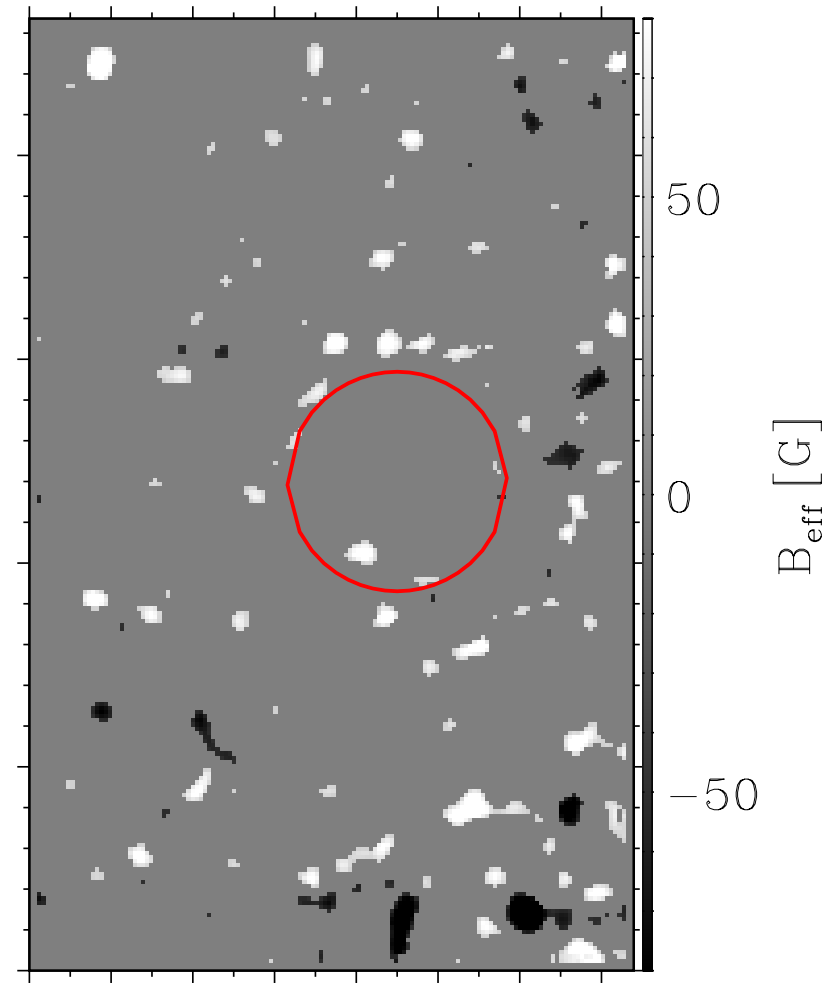

\subsubsection{Mesogranulation}

A representation of only the strong signals in the magnetogram shows a peculiar distribution of patches following a characteristic pattern with a size of about $5^{\prime \prime}$ (see Fig. 3.14). The scale of this pattern suggests that these magnetic signals are tracing the mesogranulation.

Mesogranulation is one of the characteristic scales of convection in-between the granulation (with sizes of $1 \mathrm{Mm}$ ) and the supergranulation (with sizes of 20$30 \mathrm{Mm}$ ). It was detected for the first time by November et al. (1981) using maps of vertical velocities. Mesogranules can also be detected in intensity images (e.g. Deubner 1989) and in maps of horizontal velocities (e.g. Hirzberger et al. 1997). This horizontal motion is usually computed with Local Correlation Tracking (LCT, see November \& Simon 1988) over a time series of intensity images. However, all these three methods employ smoothing to avoid contamination by granulation. Thus, there is resulted a large range of sizes associated with mesogranules depending on the method used by different authors. In the last years this has produced a controversy on the reality of this intermediate scale of convection (see Rieutord et. al 2000).

The use of the strong signal of polarization (as in Fig. 3.14) can help to solve this 
controversy. The method traces mesogranules directly and without smoothing of data. The existence of convection with a certain scale should produce horizontal motions of plasma which carry magnetic flux into the boundaries of each individual cell resulting in a cellular pattern in the magnetograms. Such a process is known to occur at the supergranular scale (producing the network; see Fig. 1.1) and operates also in the granular convection (see Fig. 3.10). Numerical simulations of magnetoconvection predicted this behaviour at mesogranular scale (see Cattaneo et al. 2001), and here, its first detection in magnetograms is presented (see also Trujillo Bueno 2003).

Recently, an excellent correlation between the visualization mesogranules in magnetograms and with methods based on intensity images has been demonstrated (Domínguez Cerdeña 2003).

\subsubsection{Consistency}

Many of the results shown in this chapter are novel and imply a big improvement in the understanding of the quiet Sun magnetism. This section gives a listing of arguments supporting the self-consistency of the obtained results.

1. Magnetograms were computed from the observation of two spectral lines almost simultaneously in time ${ }^{5}$. The two magnetograms are very similar (Fig. 3.7) since most of the magnetic structures appear at the same location in both images which would be difficult to conceive when the signals were due to noise.

2. Many magnetic patches have larger sizes than the resolution element of $0{ }^{\prime \prime} .5$. Again this is unlikely to occur for random noise.

3. The magnetic fields are predominantly found in intergranular lanes. Such a correlation excludes the possibility of pure noise.

4. The possibility of a misalignment of the two beams used to obtain Stokes $V$ (Section 3.3.1) was tested. This kind of contamination should appear in places with strong gradients in the intensity or the velocity. A shift in the beams results in subtraction of spectral lines from different points on the solar surface, thus with different Doppler velocities. In this case the subtraction produces a false Stokes $V$ signal. This spurious signal is larger for stronger gradients of velocities which happen in transitions between granules and intergranules. The signals in Fig. 3.10 do not seem to stem from these particular regions. Moreover, the plot of the magnetic flux versus the gradient in different directions of the continuum intensity (and also versus gradient of the velocities) does not show any clear dependence.

\footnotetext{
${ }^{5}$ The gap between the observation of the lines is about $20 \mathrm{sec}$.
} 
5. Another source, able to produce artificial Stokes $V$ signals, can be the nonlinearity of the narrow-band detector (CCD 2). A detector has a non-linear response to the light when an increase by a factor of $N$ in the number of photons does not result in a factor of $N$ more counts on it. In this case, a different illumination on the two parts of the CCD (from the two circularly polarized beams) results in a false signal of polarization. To check that such an effect is not present, images were taken from the same source (quiet Sun region) with different exposure times yielding the usual range of counts. The plot of counts (average over the FOV) versus exposure time shows a quasi perfect linear slope. We may thus reject the non-linearity as a source of error.

6. A possible source of error may have its origin in the crosstalk. The influence of the crosstalk from Stokes $I$ becomes negligible for the technique used in the computation of the magnetic field (see Section 3.3.3). However, the crosstalk from linear polarization (Stokes $Q$ and $U$ ) can not be corrected in this case. The estimation of the crosstalk coefficients $m_{Q}$ and $m_{U}$ for the FPI yields some few per cent (see Section 3.3.2), while for the VTT they are not larger than 10-20\%. Since the magnetic fields present in the quiet Sun have predominantly vertical direction the polarization signals in Stokes $Q$ and $U$ are smaller than the signals in Stokes $V$. Thus the crosstalk from linear polarization hardly affect to the results.

7. A glance at the temporal evolution of the magnetograms (Fig. 3.12 and 3.13) shows the presence of many magnetic patches remaining in the same place from one snapshot to the next. This is new evidence for the consistency of the results. Such a behavior can not be associated with noise.

\subsubsection{The effect of the seeing}

The observations presented above have supplied a new lower limit of the unsigned magnetic flux density measured in the quiet Sun (Eq. 3.27). The value exceeds all previous measurements, for the main reason of improved spatial resolution.

To compare with non-restored observations one must take into account how well the speckle reconstruction performs. While slit spectropolarimetric observations are affected by the seeing conditions ${ }^{6}$, the speckle reconstructed images are cleaned from most of the effects produced by the atmosphere and the telescope. Thus, one can state that restored images are closer to reality than non-restored ones. A good demonstration can be obtained by smearing the observations. Instead of using the optimum filter $H$ in Eq. 3.10, a seeing-like filter is applied

\footnotetext{
${ }^{6}$ Until now there are no previous observations of IN with adaptive optics or any other kind of restoration.
} 


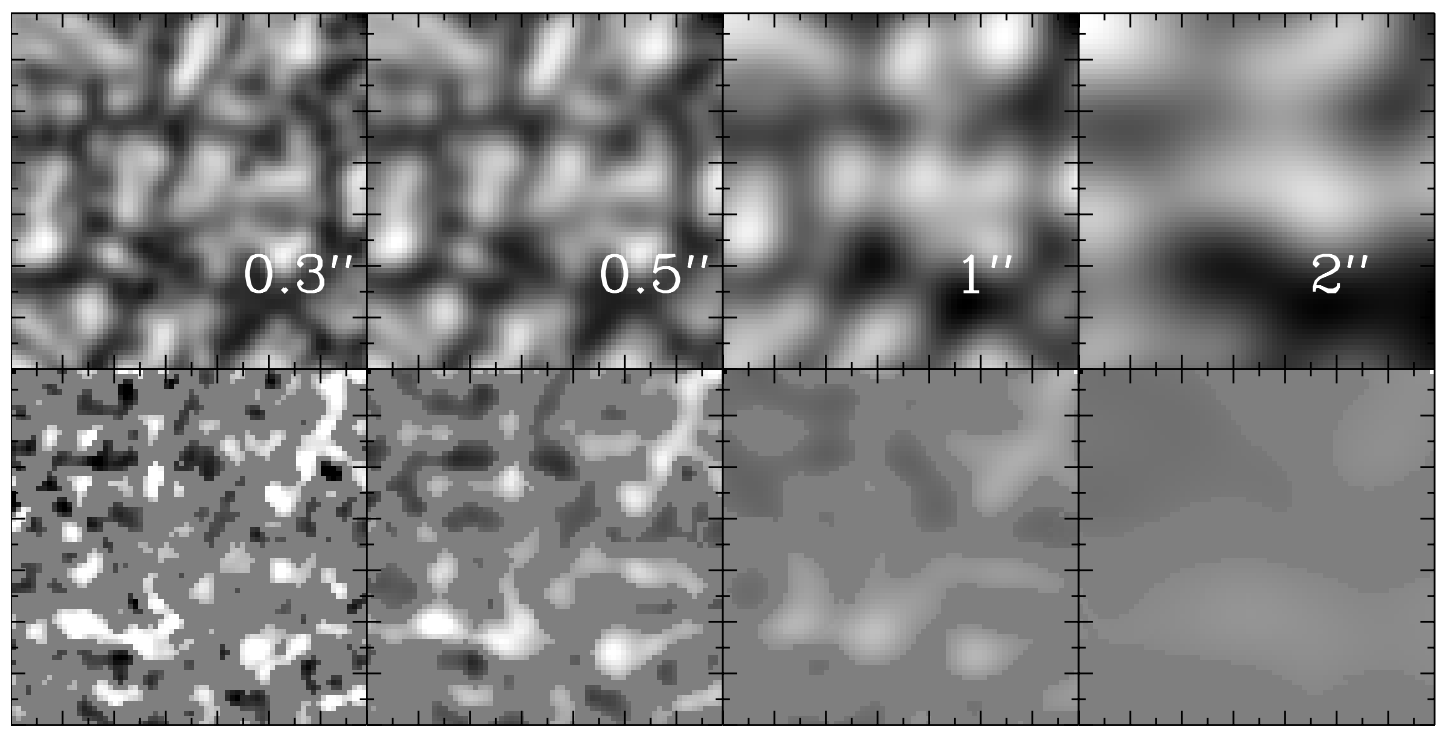

Figure 3.15: Effect of four different seeing conditions on intensity images (top) and the magnetograms (bottom). All magnetograms are equally scaled to 60 and $-60 \mathrm{G}$. The FOV is $7^{\prime \prime} \times 7^{\prime \prime}$.

to the data at this step of restoration. As it is shown in Fig $3.5 \mathrm{a}$, the use of a Gaussian filter corresponding to a seeing of 0.5 suppresses much more power from the images than the optimum filter. This means that a usual image affected by seeing has less spatial information than a reconstructed one with the same "resolution" parameter. Thus, in the case of magnetograms from quiet Sun (with unresolved magnetic structures), restored data yield more flux than nonrestored data. Figure 3.15 shows the same $7^{\prime \prime} \times 7^{\prime \prime}$ FOV under different synthetic seeing conditions, with intensity images in the upper row and magnetograms in the lower row. The FWHM (Full Width at Half of Maximum) of the seeing filter was set to $0^{\prime \prime} \cdot 3^{7}, 0^{\prime \prime} .5,1^{\prime \prime}$ and $2^{\prime \prime}$. The intensity image with $1^{\prime \prime}$ resolution exhibits a contrast of $3.6 \%$. The same contrast was obtained from slit observations with $1^{\prime \prime}$ resolution by Lites (2002) for the same spectral line. This is an evidence for the consistency of the smearing procedure, since the relation between the contrast and spatial resolution can be considered linear except for higher resolution than $0 \prime .5$. All magnetograms in the figure are equally scaled for comparison. There is a huge difference between magnetograms with 0.5 resolution and $1^{\prime \prime}-2^{\prime \prime}$ (seeing conditions for typical observations), not only in the loss of richness of structures but also in the loss of flux as the magnetic signals are much weaker in the latter magnetograms.

\footnotetext{
${ }^{7}$ This "higher" resolution with regard to the cutoff selected in Section 3.3.1 should not be confusing, since the speckle reconstructed image contains the original power.
} 


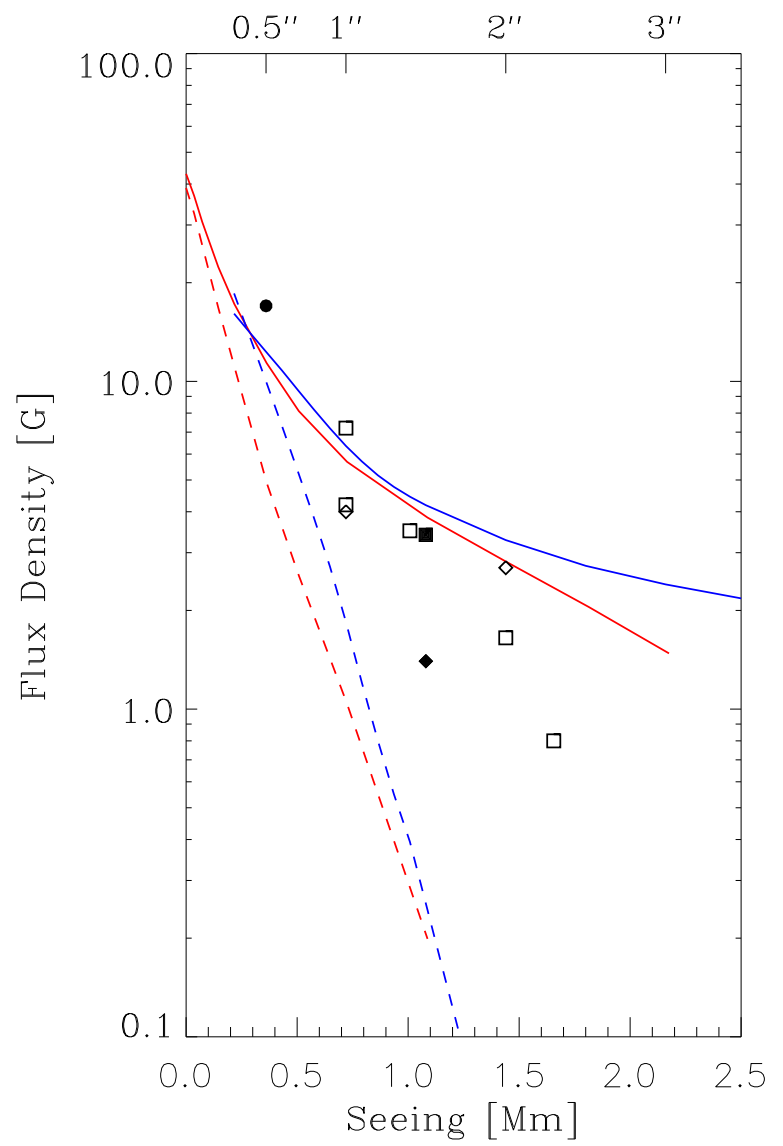

Figure 3.16: Unsigned flux density vs. seeing condition. Blue curves: Flux from $\mathrm{Fe}$ I $\lambda 6302.5 \AA$ magnetograms computed from data restored with a seeing filter (see Fig. 3.5a). Red curves: Flux density from magnetoconvection simulations smeared with different spatial resolutions. Both cases present fluxes with maximum sensitivity (solid lines) and with a sensitivity of $20 \mathrm{G}$ (dashed lines). Symbols: Flux from observations, this work (•, from Eq. 3.27), works using visible lines $(\square)$ and using IR lines $(\diamond)$, and from the simultaneous observations in Chapter 4 $(\boldsymbol{\square}$ and $\boldsymbol{\vee}$, from Eq. 4.2) . See Table 3.1.

The blue curve in Fig. 3.16 displays the unsigned flux remaining, after smearing with different seeing parameters in the data from this work. The decrease of magnetic flux is due to cancellation between patches of opposite polarity. The fluxes were computed for pixels with signal above noise, resulting that the occupation over the FOV was $\sim 50 \%$. Even when polarization signals have become weaker from smearing the percentage of pixels above noise was almost independent of the seeing parameter, since the noise decreases also with larger smearing. Figure 3.16 also displays the flux computed for pixels above a fixed threshold of 20 G (dashed blue), which exhibits a deeper fall. Sánchez Almeida et al. (2003b) collected unsigned flux obtained in previous works (see Table 3.1) with the same behavior of decreasing flux density with spoiling spatial resolution. Its values are plotted in Fig. 3.16 for visible (squares) and IR (diamonds) observations. In the comparison, one must take into account the fractional area considered in the calculation of the flux (see Table 3.1). Visible observations tend to have less sensitivity than the data smeared by seeing of this work, thus yielding fluxes below the blue curve. The exception is the flux density obtained by Lites (2002) with $f$ of 0.60 and an unsigned flux just above the curve. On the other hand IR observations have high sensitivity but measure lower fluxes. Finally, the dot represents 
Table 3.1: Values of averaged unsigned flux density and spatial resolutions from previous works (collected by Sánchez Almeida et al. 2003b).

\begin{tabular}{lcccr}
\hline \hline & & & & \\
source & resolution & $\left|B_{\text {eff }}\right|$ & $f$ & spectral lines \\
\hline this work & $0^{\prime \prime} 5$ & 17 & 0.45 & Fe I $\lambda 6302.5 \AA$ \\
Lites (2002) & $1^{\prime \prime}$ & 7.2 & 0.60 & Fe I $\lambda 6302.5 \AA$ \\
Socas-Navarro & & & & \\
$\quad$ \& Sánchez Almeida (2002) & $1^{\prime \prime}$ & 4.3 & 0.27 & Fe I $\lambda 6302.5 \AA$ \\
Khomenko et al. (2003) & $1^{\prime \prime}$ & 4 & 0.50 & Fe I $\lambda 15648 \AA$ \\
Stolpe \& Kneer (2000) & $1^{\prime \prime} .4$ & 3.5 & 0.25 & Fe I $\lambda 6302.5 \AA$ \\
Chapter 4 & $1^{\prime \prime} .5$ & $3.4^{a}$ & 0.45 & Fe I $\lambda 6302.5 \AA$ \\
Chapter 4 & $1^{\prime \prime} .5$ & $1.4^{a}$ & 0.45 & Fe I $\lambda 15648 \AA$ \\
Lin \& Rimmele (1999) & $2^{\prime \prime}$ & 2.7 & 0.68 & Fe I $\lambda 15648 \AA$ \\
Wang et al. (1995) & $2^{\prime \prime}$ & 1.65 & 0.35 & BBSO magnetogram \\
Grossman-Doerth et al. (1996) & $2^{\prime \prime} .3$ & 0.8 & 0.40 & Fe I $\lambda 5250 \AA$ \\
\hline \hline
\end{tabular}

${ }^{a}$ From magnetograms of simultaneous observations (see Section 4.3).

the flux density obtained from the speckle reconstructed data, which is larger than expected from the blue curve as it was discussed before. The red curves present the flux density obtained from numerical simulations of a local magnetic dynamo (Cattaneo 1999) versus different seeing conditions (see Sánchez Almeida et al. 2003b). The curve for flux density with maximum sensitivity (solid) has a similar shape as the observations at high resolution. However, simulations are well balanced in magnetic flux, thus the decrease in resolution leads to a limit of zero flux, while real observations are imbalanced and the corresponding curve tends to $\sim 2 \mathrm{G}$ (see Section 3.4.1).

The study of flux density versus spatial resolution can give an idea on the importance of the unsigned flux that should be observed in the quiet Sun with infinite angular resolution. Local dynamo simulation gives a value of $\sim 50 \mathrm{G}$ (see Sánchez Almeida et al. 2003b). However, this value can be larger. When one compares the curves with the same polarimetric sensitivity of $20 \mathrm{G}$ (dashed lines in Fig. 3.16) obtained from observations (blue) and from simulations (red), one finds a tendency of higher flux density in the observations than in the simulations. It thus seems that the IN carries more flux than expected from simulations. 


\section{Chapter 4}

\section{Simultaneous visible and infrared observations}

\subsection{Observations: Spectropolarimetry with two telescopes}

The data presented in this Chapter stem from the first ever simultaneous visible and infrared (IR) spectropolarimetry. Such an observation needs the use of two telescopes ${ }^{1}$ and was only possible at the Spanish Observatorio del Teide (Tenerife, Spain). This observatory operates two leading solar telescopes with sensitive spectropolarimeters. The IR observations were gathered with the Tenerife Infrared Polarimeter (TIP; Collados 1999; Martínez Pillet et al. 1999) operated at the German Vacuum Tower Telescope (VTT). Spectrograms of the full Stokes vector $(I, Q, U, V)$ were obtained in the IR iron lines Fe I $\lambda 15648 \AA$ (Landé factor $\left.g_{L}=3\right)$ and $\mathrm{Fe}$ I $\lambda 15652 \AA\left(g_{L}=1.53\right)$. The visible observations were carried out with the French-Italian telescope THEMIS (Téléscope Heliographique pour l'Étude du Magnétisme et des Instabilités Solaires) using the spectropolarimetric mode MTR (Mein \& Rayrole 1985; Rayrole \& Mein 1993). Three different visible spectral ranges were observed simultaneously with the MTR, however only one is under study in this thesis. The lines observed are those studied in Chapter 3, the pair of iron lines Fe I $\lambda 6301.5 \AA$ and Fe I $\lambda 6302.5 \AA$. The atomic parameters of the four observed lines are listed in Table 4.1.

The instruments used are slit spectropolarimeters which allow to measure onedimensional spectra. Using different modulations of a polarization analyzer it is possible to measure spectra from which one can obtain the four Stokes parameters (see Section 4.2). The slit width was set to 0.5 in both telescopes. To obtain two-dimensional fields of view (FOV) the solar surface was scanned with the

\footnotetext{
${ }^{1}$ Nowadays it is possible with TIP+POLIS (VTT, Tenerife) and SPINOR (DST, Sac Peak)
} 
Table 4.1: Atomic parameters of the lines studied.

\begin{tabular}{lcccc}
\hline \hline Wavelength $(\AA)$ & $\chi(\mathrm{eV})^{a}$ & $A g f^{b}$ & Transition $^{c}$ & $g_{L}$ \\
\hline $6301.499^{d}$ & 3.65 & $9.766 \cdot 10^{-6}$ & ${ }^{5} \mathrm{P}_{2} \rightarrow{ }^{5} \mathrm{D}_{2}$ & 1.67 \\
$6302.492^{d}$ & 3.69 & $2.630 \cdot 10^{-6}$ & ${ }^{5} \mathrm{P}_{1} \rightarrow{ }^{5} \mathrm{D}_{0}$ & 2.5 \\
$15648.515^{e}$ & 5.426 & $8.035 \cdot 10^{-6}$ & ${ }^{7} \mathrm{D}_{1} \rightarrow{ }^{7} \mathrm{D}_{1}$ & 3 \\
$15652.874^{e}$ & 6.246 & $3.443 \cdot 10^{-5}$ & ${ }^{7} \mathrm{D}_{5} \rightarrow{ }^{7} \mathrm{D}_{4}$ & $1.53^{1}$ \\
\hline \hline
\end{tabular}

${ }^{a}$ Excitation potential from Moore, Minnaert, \& Houtgast 1966, and Kurucz 1994.

${ }^{b}$ Abundance of Fe, i.e. total Fe times statistical weight of the lower level times oscillator strength from Gurtovenko \& Kostic 1981 (visible lines) and from Borrero et al. 2003 (IR lines).

${ }^{c}$ Levels which take part in the transition from Nave et al. 1994.

${ }^{d}$ Wavelengths from Higgs 1962.

e Wavelengths from Nave et al. 1994.

telescopes from East to West taking spectrograms at 60 positions separated by $0 \prime 5$. In order to reduce the noise, the integration time was $30 \mathrm{~s}$ per position, yielding a duration of 30 minutes for one scan.

The data set of this work was observed on 2002, August 10 in an intra-network (IN) region close to disk center $(\mu=0.94)$. To select quiet Sun regions one avoids bright features in $\mathrm{Ca}$ II $\mathrm{K}$ and $\mathrm{H} \alpha$ slit-jaw images from video cameras during observation. In order to point both telescopes to the same target on the Sun, a video link between both telescopes was used providing the slit-jaw images from VTT to THEMIS.

Since the integration time is long and the variation of granulation is fast (on the order of minutes) one needs to point the slit in both telescopes to the same region with an offset less than few arcsec. Since the pointing of the telescopes (using heliographic coordinates) is not accurate enough, and the slit-jaw images of the granulation are not a feasible way to recognize the region pointed by the two telescopes one needs another method for coordinated pointing. The method used was a kind of differential pointing. First, both telescopes are pointed to a characteristic small structure which can be easily identified, e.g., a small pore. Then the VTT pointing was changed to a quiet Sun region, and finally the THEMIS pointing was displaced by the same distance.

The differential refraction was also corrected. When the Sun is low in the sky, the light is refracted more in the visible than in the IR. For the day of observation, the offset due to differential refraction amounted to $3^{\prime \prime}$ at 8:00 UT. Three of the five trials to observe the same region had a shift of $5^{\prime \prime}$. However, the one under study here had an offset between the telescopes of only $1^{\prime \prime}$. When this shift is corrected (see Section 4.2.2), it corresponds to a time lag between the IR and 
visible data of 1 minute which can be considered simultaneous enough for the study of the IN fields.

The spatial resolution of the IR observations is $1^{\prime \prime}-1$ ".2 with a granular intensity contrast of $1.7 \%$ while it becomes 1.5 in the visible range with a contrast of $2 \%$. The cutoff of the power spectra of the continuum images was the tool used to estimate the resolution. The estimated resolution and contrast in the visible spectral range agree with those obtained from the smearing of speckle reconstructed images at the same wavelength (Section 3.4.7). Figure 4.2 shows continuum images from both spectral ranges. The better resolution of the IR data is due to the observation with a correlation tracker in the VTT using an active tip-tilt mirror which corrects global image motions during the scanning (Ballesteros et al. 1996).

\subsection{Data reduction}

TIP uses a polarization analyzer consisting of two ferro-electric liquid crystals followed by a calcite beam-splitter. The instrument gathers four linear combinations of Stokes parameters every $0.5 \mathrm{~s}$. In order to have an integration time of 30 s per slit position one takes 60 accumulations of this kind. The calcite produces two beams from which two spectrograms are formed on separate parts of the CCD. The simultaneous measurements of the two beams avoids seeing-induced crosstalk $^{2}$.

The polarization modulator of the MTR mode of THEMIS is formed by two retarders (quarter-wave plates), and also a calcite beam splitter. Depending on the combination of angles of the retarders (EM25 and EM26) one can obtain different combinations of Stokes parameters. Table 4.2 shows the 4 measurements

Table 4.2: Modulations at THEMIS with the angles of the quarter-wave plates (EM 25 and EM 26) used for each measurement. The angles are measured clockwise relative to the direction of the polarization of the calcite ordinary beam.

\begin{tabular}{ccc}
\hline \hline EM 25 & EM 26 & Measurement \\
\hline$-45^{\circ}$ & $0^{\circ}$ & $I \pm V$ \\
$+45^{\circ}$ & $0^{\circ}$ & $I \mp V$ \\
$0^{\circ}$ & $0^{\circ}$ & $I \pm Q$ \\
$+22.5^{\circ}$ & $+22.5^{\circ}$ & $I \pm U$ \\
\hline \hline
\end{tabular}

\footnotetext{
${ }^{2}$ Artificial polarization signals produced by the variation of the Stokes profiles due to the motion of the image produced by atmospheric perturbations.
} 
performed in the present observation. $I \pm V$ means that the one part of the CCD detector gathers $I+V$ and the other part $I-V$. As in TIP, the purpose of such measurements is the correction of the seeing-induced crosstalk. In the case of THEMIS, the series of modulations in Table 4.2 are measured every $6 \mathrm{~s}$, and 5 accumulations were gathered per slit position giving also a total integration of $30 \mathrm{~s}$.

Each of the two beams observed is a spectral image with a size in the spectral direction of 256 pixels for TIP and 280 pixels for THEMIS with a sampling of $29.1 \mathrm{~m} \AA$ and $22 \mathrm{~m} \AA$, respectively. The size in the spatial direction was 101 pixels for TIP (corresponding to a total length of $35^{\prime \prime}$, see Section 4.2.2) and 130 for THEMIS (equivalent to $60^{\prime \prime}$ ). Hence there are 101 and 130 individual spectrum lines per spectral image in the visible and IR spectral range, respectively.

The data reduction was done in the usual way with similar procedures for both data sets:

- To remove the differential response of the CCD to the light, one must correct with flat fields. The corresponding images were taken with both telescopes by moving telescope pointing over a quiet Sun region thereby avoiding the magnetic regions. To clear out the flats from any solar structure many images were averaged to get a flat for each modulation step. To have one flat per modulation will remove many interference fringes produced by the optics of the instruments.

- There are some interference fringes with a time depending position on the detectors. These were removed by measuring two flats, one before and one after each observation set. A linear interpolation in time between these two flat field images corrects most of the fringes.

- Spectrograms from flat fields and observed data were corrected for inclination of the lines in direction of dispersion and for differential spectral dispersion. To correct these effects each individual spectrum must be displaced with a precision of a hundredth of pixel. Many interpolation methods were tested and the most effective turned to be the Fourier interpolation (taking advantage of the "shift theorem" for Fourier transforms). Due to small vibrations of the detector of THEMIS (produced by the mechanical motions during the modulation) there is a global shift from one spectral image to the next which was also corrected. These artifacts must be removed from both flat field images and data spectral images.

- After flat field correction, the measurements are demodulated to obtain the four Stokes parameters. The residual interference fringes are removed by fitting them to a series of harmonics (sinus and cosinus). Finally the two beams are combined to remove the seeing-induced crosstalk. In the case of 
TIP the demodulation matrix must be calculated with measurements using calibration optics in front of the polarization analyzer.

- The crosstalk ${ }^{3}$ from $I$ to $Q, U$ and $V$ was removed by setting each Stokes parameter to zero at the wavelengths corresponding to the continuum of the lines in the following way

$$
X=X^{\prime}-m_{I \rightarrow X} I
$$

where $m_{I \rightarrow X}$ is the crosstalk coefficient obtained from the value of Stokes $X^{\prime}$ in the continuum (where $X$ is expected to be zero).

- Finally the crosstalk produced by the instrumental polarization (IP) of the telescope itself was removed. Since THEMIS is free of IP, the visible data do not need this kind of correction. The correction of IP in the VTT was possible with the calculation of the Mueller matrix for the telescope. It was calibrated with measurements with different orientation of the axis of a linear polarizer mounted at the coelostat and at the entrance window of the evacuated tube of the telescope (see Collados 2003).

More details about the instruments and the data reduction can be found in Collados (1999) and Schlichenmaier \& Collados (2002) for TIP, and in López Ariste et al. (2000) and Bommier \& Rayrole (2002) for THEMIS.

\subsubsection{Wavelength calibration}

An absolute wavelength calibration was done. Since the data used have lines in the visible and in the IR and the final purpose of this work is the simultaneous inversion of the four lines, one needs to have a consistent calibration in both spectral ranges.

For the visible lines the central wavelength of Fe I $\lambda 6301.5 \AA$ and Fe I $\lambda 6302.5 \AA$ was considered to be the zero-crossing of the Stokes $V$ profiles corresponding to the strongest signals in the network points from the FOV (see Fig. 4.5). Such a method was tested in the past by Solanki (1986) giving an accuracy of $100 \mathrm{~m} \mathrm{~s}^{-1}$ $(2 \mathrm{~m} \AA)$. Only the strongest signals were chosen $\left(V>0.02 I_{c}\right.$ with $I_{c}$ the intensity of the continuum near the line). Weaker signals tend to be redshifted as it is shown in Fig. 4.1. The calibration yields also the spectral sampling, i.e. the pixel distance in dispersion, of $21.94 \mathrm{~m} \AA$.

Since this method has not been tested for the IR lines they need another way of calibration. A mean Stokes $I$ profile over the FOV was computed. The minimum of the lines was fit to a fourth order polynomial whose minimum position was

\footnotetext{
${ }^{3}$ Contamination in one Stokes parameter from the others, see Section 3.3.2.
} 

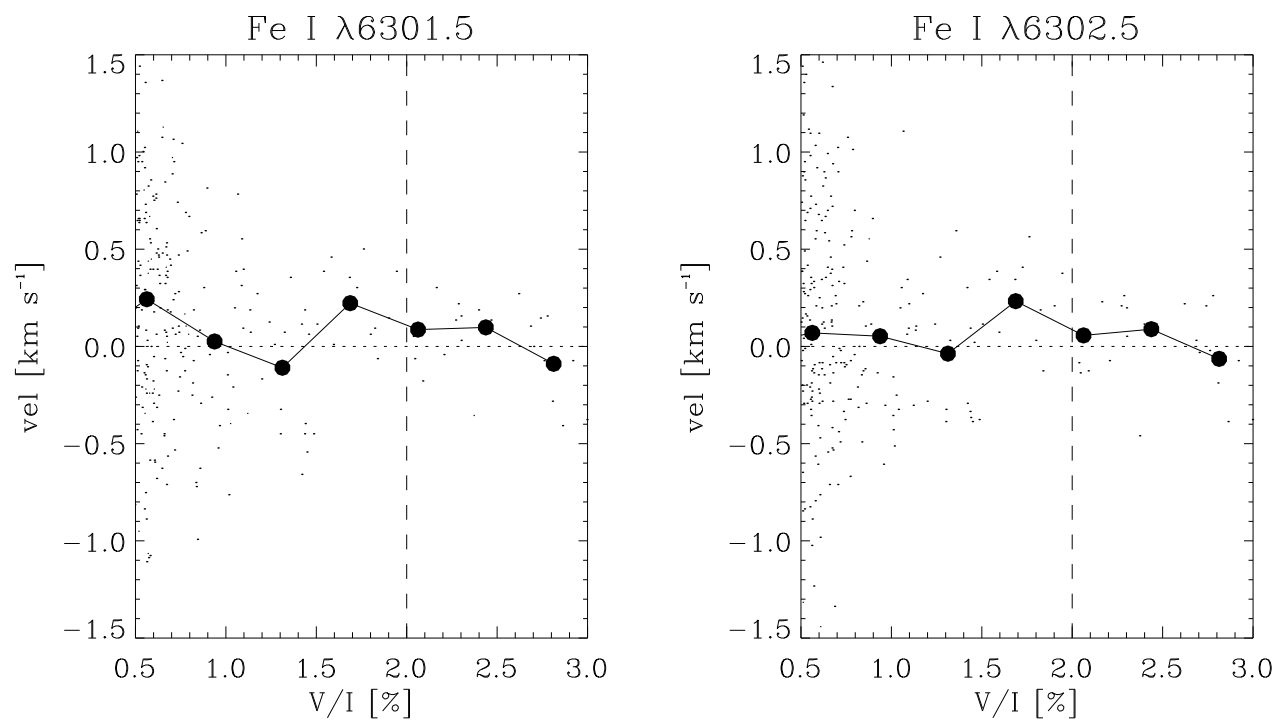

Figure 4.1: Zero-crossing velocities computed for Stokes $V$ profiles versus the level of polarization for Fe I $\lambda 6301.5 \AA$ (left) and Fe I $\lambda 6302.5 \AA$ (right). Dashed vertical line represents the threshold chosen to calibrate. Filled circles represent means of velocities for different bins of level of polarization to show the redshift tendency. The convention here is that positive velocities are defined as redshift.

taken as the central wavelength of the spectral line. To make this calibration absolute one must correct for the convective blue shift. Table 4.3 presents the convective blue shift for Fe I $\lambda 15648 \AA$ and Fe I $\lambda 15652 \AA$ obtained from a twocomponent inversion of many line profiles from quiet Sun (Borrero et al. 2003) and from line synthesis from numerical simulation of granulation (Asplund et al. 2000).

Table 4.3: Convective blue shift for the four spectral lines from two sources.

\begin{tabular}{ccc}
\hline \hline line & $v_{C B}{ }^{a}\left(\mathrm{~m} \mathrm{~s}^{-1}\right)$ & $v_{C B}{ }^{b}\left(\mathrm{~m} \mathrm{~s}^{-1}\right)$ \\
\hline Fe I $\lambda 6301.5 \AA$ & +50 & -100 \\
Fe I $\lambda 6302.5 \AA$ & -80 & -150 \\
Fe I $\lambda 15648 \AA$ & -370 & -400 \\
Fe I $\lambda 15652 \AA$ & -430 & -400 \\
\hline \hline
\end{tabular}

${ }^{a}$ From synthesis of simulations by Asplund et al. 2000

${ }^{b}$ From two-component inversion of many iron lines (Borrero et al. 2003; Borrero \& Bellot Rubio 2002) 


\subsubsection{Alignment and spatial resolution}

One of the most important steps in the data reduction is the spatial alignment of the visible and IR scans. The aim of this process is to ensure that the scans are not only taken from the same region on the Sun but also that each individual pixel has the information (full Stokes spectra) for the visible and IR lines from the same point of the IN region. The correct alignment must be guaranteed for a meaningful analysis of the data, i.e. for the comparison of the results from both spectral ranges and the simultaneous interpretation of both data sets.

To perform correctly this procedure one needs to take into account five parameters, the scales of the data sets in $X$ and $Y$ direction, the shift in $X$ and $Y$ direction and the rotation of the data sets with respect to each other.

- Rotation. The orientation of the spectrograph slits in the two telescopes was not exactly the same. In THEMIS the slit was always in the NorthSouth solar direction. However, the slit in the VTT was fixed at $12^{\circ}$ from North-South Earth orientation at the beginning of the observing campaign. During the observing day the North-South solar axis had an inclination of $13^{\circ}$ with the North-South Earth axis, resulting in a correction of $1^{\circ}$ with an error of $\pm 10^{\prime}$.

- Scales. The pixel size of the two data sets is different.

The $X$ direction is the direction of scanning perpendicularly to the slit (the horizontal direction in Fig. 4.2). In THEMIS this scale was 0.5 per pixel, but due to mechanic limitations the scale at the VTT was 0.'515 per pixel.

The continuum image of a sunspot, from data gathered the same day, was used to calibrate the scale in the $Y$ direction (the spatial direction of the spectral images). On the one hand the spatial size of the spectra in THEMIS is given by the size of the pre-slit, $60^{\prime \prime}$. A comparison with MDI continuum images shows that this value is correct, yielding a pixel size of $0{ }^{\prime \prime} 465$. The IR image of the sunspot was correlated with the image in the visible, resulting in a pixel size for TIP data of 0"'35 (a bit lower than the 0!'38 observed by Khomenko et al. 2003)

- Shift. There is a spatial shift of the scans due to the uncertainties in the pointing of the telescopes (explained in Section 4.1).

Once the rotation and the scales were corrected, it was relatively easy to calculate via correlation in Fourier space the shift in $X$ and $Y$ direction, using just the intensity images (from the continuum near the spectral lines). The visible and IR images were very similar when the IR image was smeared to the resolution of the visible one (see Fig. 4.2). In the $Y$ direction the visible map covers the whole IR map, and finally the shift in $X$ direction was only 0 "' $6(\sim 1$ pixel $)$. 

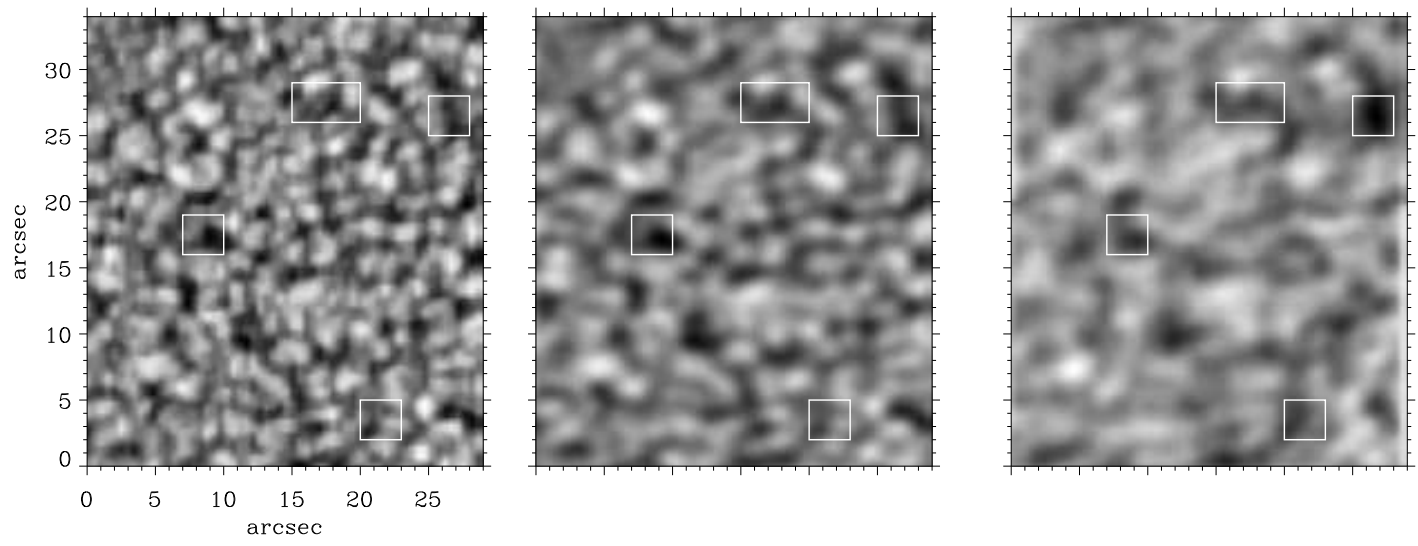

Figure 4.2: Intensity images in the continuum of the IR lines before (left) and after smearing (middle) and of the visible lines (right). The squares point out structures that can be identified very easily in both IR and visible images. Axes in arcsec.

The parameters thus obtained were used to align the visible data to the IR data. The visible spectrograms were computed as an average, with appropriate weights, over four pixels of the THEMIS scan such that the resulting positions correspond to those in the TIP scan. Figure 4.2 shows the good alignment between the two images in IR (left) and visible (right). Squares show structures easily recognized in both maps. Overall, the FOV alignment was better than half a pixel. The final FOV of the data was $30^{\prime \prime} \times 34^{\prime \prime}$.

In a simultaneous study of the visible and IR data it is mandatory to have the same resolution for both data sets. However, the angular resolution of the observations at the VTT was better than at THEMIS. To solve this problem the IR data were degraded to the resolution of the visible data (1".5). The center panel in Fig. 4.2 shows a continuum image in the IR after smearing. The contrast of the IR image is now $0.9 \%$.

A possible way to check that the resolution is now the same for both observations is to compare them with synthesized images obtained from three-dimensional numerical simulations of granular convection. Such simulations were carried out with the MURAM code by Vögler \& Schüssler (2003) and Vögler (2003). The images in the left panels in Fig. 4.3 represent calculated continuum intensities at $\lambda 6302.5 \AA$ (top) and at $\lambda 15648 \AA$ (bottom) from these simulations (see Shelyag et al. 2003). They were smoothed to the resolution of the data (in a similar way as in Schüssler et al. 2003) and are shown in the middle panels of the figure. Finally the right panels show a fragment of continuum images from the observations in the two spectral ranges and with the same size as the simulations $\left(6 \times 6 \mathrm{Mm}^{2}\right.$ or 

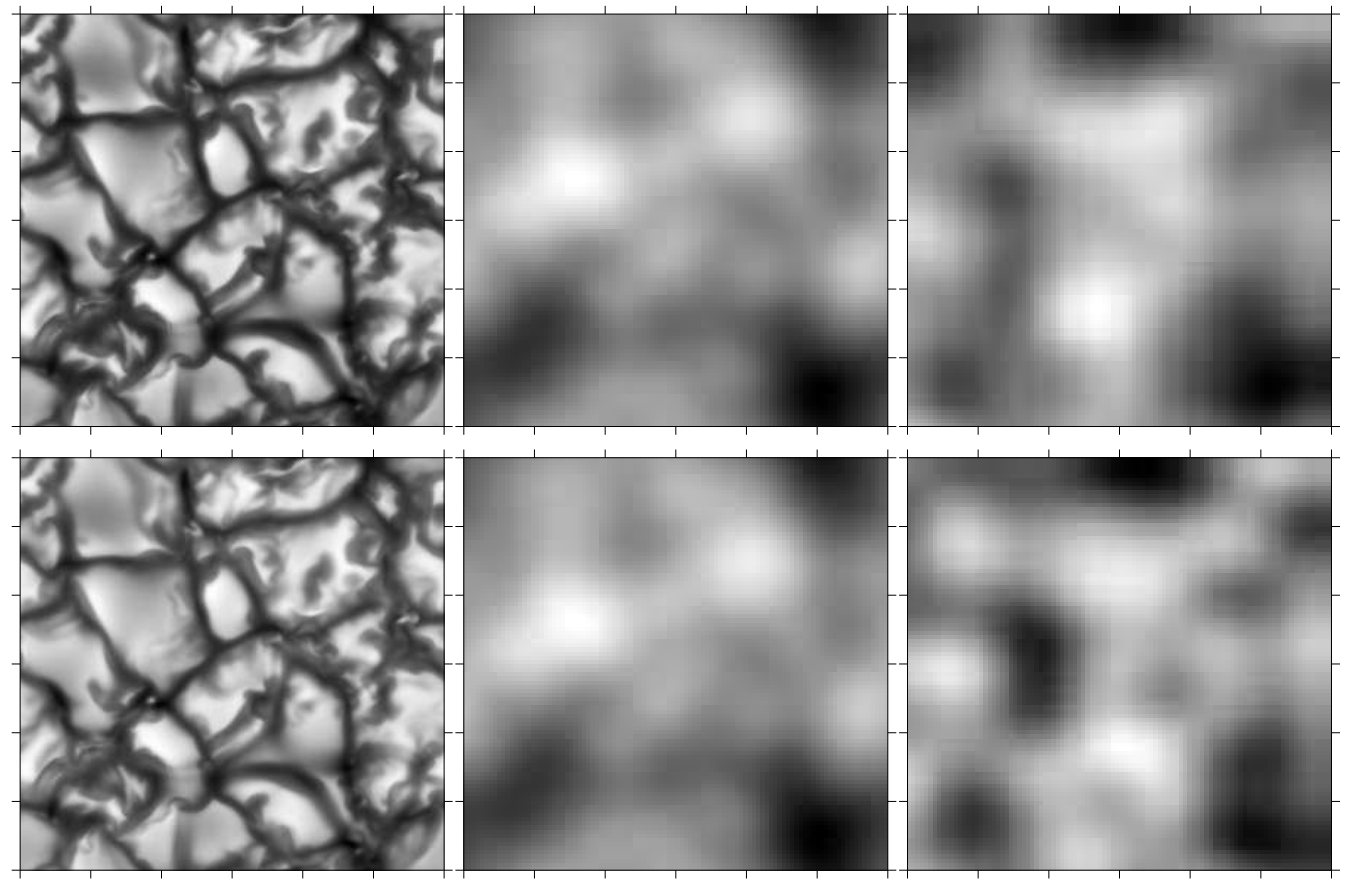

Figure 4.3: Comparison of observation with synthetic images from simulations. Images synthesized for continuum of $\lambda 6302.5 \AA$ (top) and at $\lambda 15648 \AA$ (bottom) from 3D numerical simulations of granulation (left panels, courtesy S. Shelyag, Max-Planck-Institut für Aeronomie). The same images after smearing to 1".5 spatial resolution (middle) are compared with a fragment from images of the observations (right). Separation between tickmarks is in Mm.

$8.3 \times 8.3)$. Neglecting that the simulation and the observation contain different samples of granulation, both images look quiet similar.

Figure 4.4 shows a scatter plot of intensity in IR $v s$. intensity in visible, for observations (blue) and simulations (red). The fit to a straight line yields in both cases a very similar slope of about 0.52 . This is an argument to believe that both observations have the same resolution. In fact any smearing of the synthetic images results in a very similar slope. It does not depend on the resolution. The contrast computed for both synthetic images was very close to the contrast of the continuum images. Note that the representation of the synthetic images gives also a cloud of points, and the larger dispersion for the observations can be due to noise and some differences in variable seeing effects between the two telescopes.

After all data reduction including smearing of the IR data and Fourier filtering of the visible data the level of noise in polarization for Stokes $V$ is $(2-3) \times 10^{-4} I_{c}$ in the IR data and $6 \times 10^{-4} I_{c}$ in the visible data ( $I_{c}$ is the continuum intensity). 


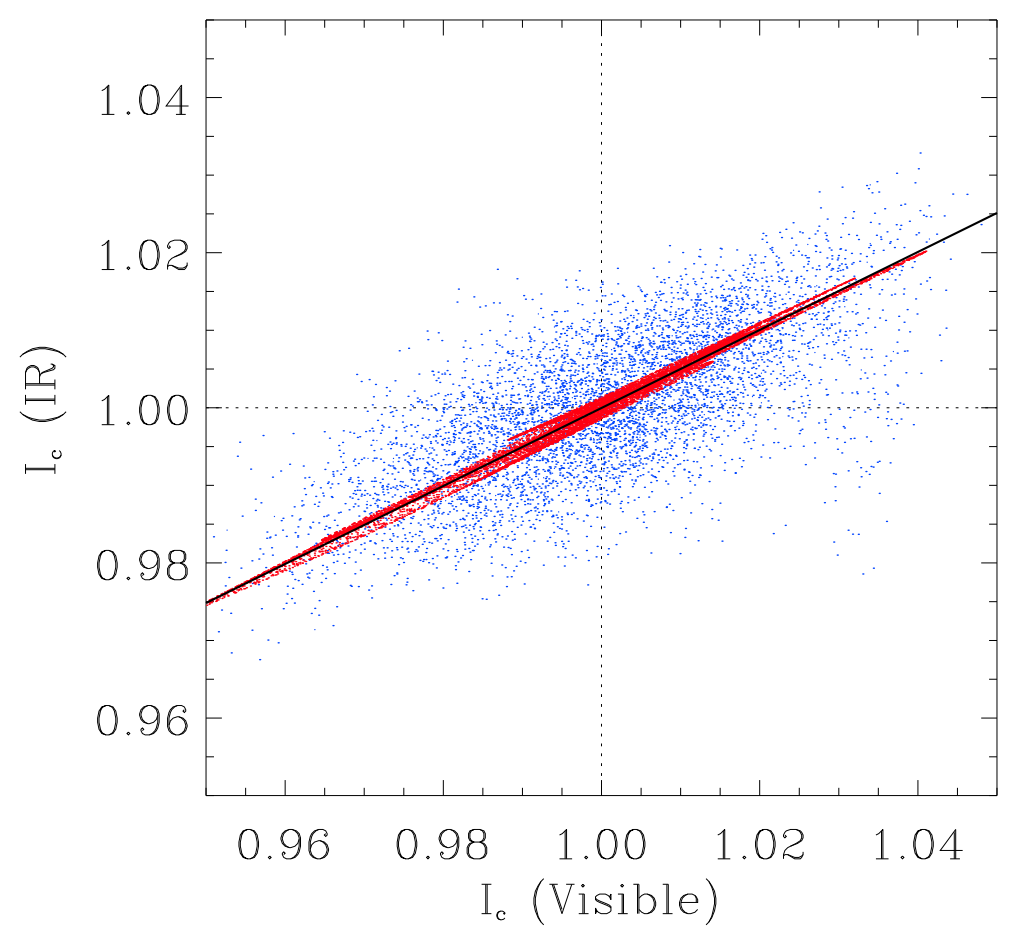

Figure 4.4: Scatter plot of the intensity in the IR range and in the visible for the observations (blue) and for the synthetic images after smearing (red). Black solid line shows the behaviour of both clouds of points.

\subsection{Magnetograms}

Figure 4.5 shows the magnetograms calculated from Stokes $V$ profiles of Fe I $\lambda 6302.5 \AA$ and Fe I $\lambda 15648 \AA$. The magnetic flux density or $B_{\text {eff }}$ was computed with the magnetograph equation under the weak field approximation as described in Section 3.3.3. Both magnetograms are scaled to the same limits of magnetic flux densities. At first sight, both images are similar with the exception that the signals found in visible are larger than signals found in IR. Indeed this is the first important difference. The unsigned flux was computed as described in Section 3.4.1. Since only the IN fields are of interest here, the network region in the lower left part of our FOV was discarded by rejecting pixels with flux densities larger than $20 \mathrm{G}$ in visible and $10 \mathrm{G}$ in IR. The noise levels turned out to be $2 \mathrm{G}$ in visible and $1 \mathrm{G}$ in IR. Finally the unsigned flux obtained from pixels in the FOV which fulfill both requirements of flux density below the upper limit 

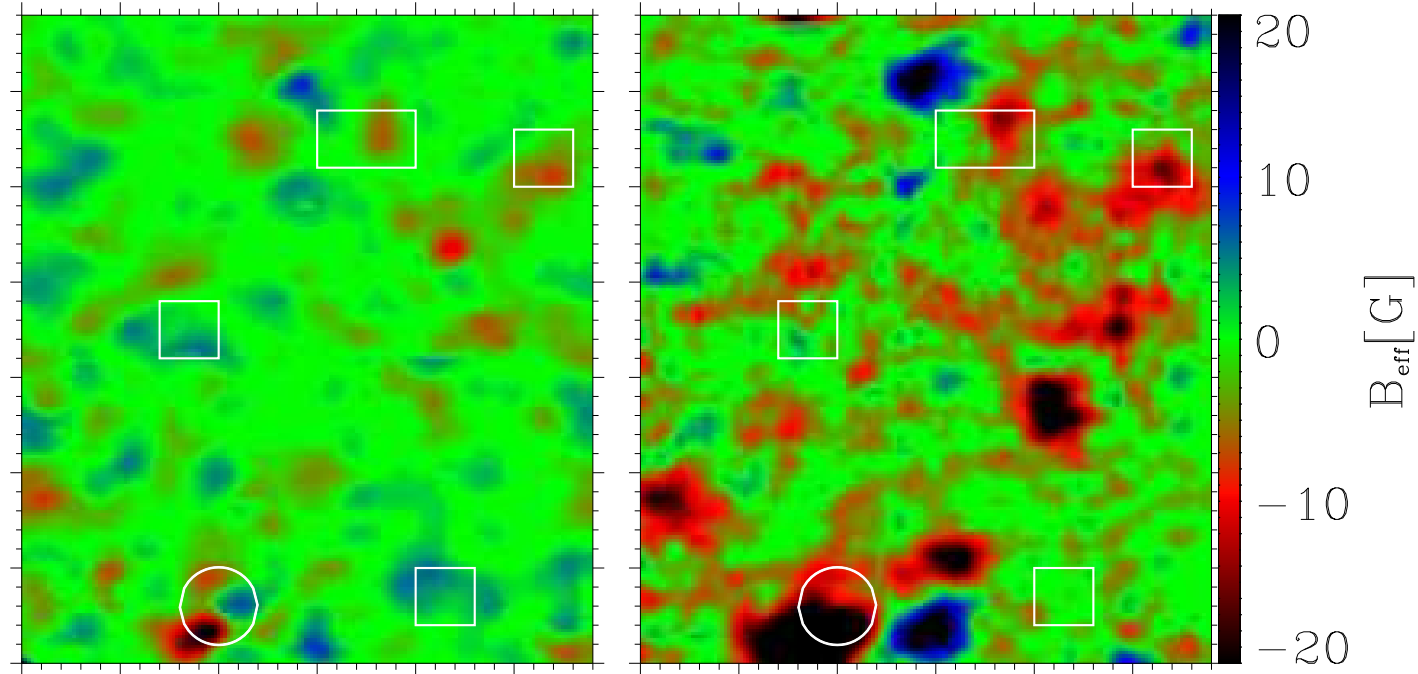

Figure 4.5: Magnetograms for Fe I $\lambda 15648 \AA$ (left) and Fe I $\lambda 6302.5 \AA$ (right). Red and blue color distinguish between polarities. Both magnetograms are scaled to flux densities $\left|B_{\text {eff }}\right|<20$ G. Distance between tickmarks is $1^{\prime \prime}$.

and above noise was

$$
\overline{\left|B_{\text {eff }}\right|}=\left\{\begin{array}{l}
3.4 \mathrm{G}, \text { for Fe I } \lambda 6302.5 \AA \\
1.4 \mathrm{G}, \text { for Fe I } \lambda 15648 \AA
\end{array}\right.
$$

In both cases the pixels taken into account covered $40-50 \%$ of the total FOV (the rest of the FOV was set to zero in the calculations). It is seen that the measurements in the visible detect more flux than in the IR. This suggests that visible and IR lines trace different magnetic structures (see Section 2.3). The difference of flux is conspicuous, and one may be tempted to ascribe it to the techniques used. However, more elaborate techniques show the same behaviour (see Fig. 4.10). One should note that the flux measured in visible lines agrees with that simulated from the speckle data smeared to 1".5 spatial resolution (see Fig. 3.16).

Magnetic flux concentrations appear very similar in some places for both magnetograms (upper squares in Fig. 4.5) but also different in some other locations (other two squares). Some structures seem to have one polarity in the IR magnetogram, and the opposite in the visible one. This effect is evident even in the network area with large polarization signals (see the circle in Fig. 4.5). Such a 
phenomenon can be considered as a further evidence of the different sensitivity to magnetic fields of the visible and IR lines.

Finally there is the appearance that magnetic fields in the right magnetogram (visible) exhibit a preferred polarity while the left magnetogram (IR) looks well balanced. The signed flux was measured to be

$$
\overline{B_{\text {eff }}}=\left\{\begin{array}{l}
-2.6 \mathrm{G}, \text { for Fe I } \lambda 6302.5 \AA \\
-0.1 \mathrm{G}, \text { for Fe I } \lambda 15648 \AA
\end{array}\right.
$$

There is a clear difference. The magnetogram computed from IR lines is well balanced while there is a large signed flux in the visible magnetogram. This imbalanced flux is similar to that observed in Section 3.4.1 and to other visible observations (e.g., Lites 2002). Yet, Khomenko et al. (2003) found almost no signed flux using the same IR lines (they observed $0.2 \mathrm{G}$ ). If the theory suggested in Section 2.3 is correct, the interpretation of Eq. 4.3 can be that weak magnetic field strengths are well balanced while $\mathrm{kG}$ fields present a clear imbalance.

In order to show that this imbalanced flux found in the visible data is not an artifact, I present a list of tests and arguments to check the consistency of this result and the data in general:

1. False signals, e.g. due to misalignment of spectrograms during data analysis, that could result in a net flux would appear in average profiles of Stokes $Q$ and $U$. The absence of net linear polarization rejects the possible effects of misalignment of the spectral images. This can also be checked with the telluric lines. There is no residue in the maps of polarization in the telluric lines confirming the good alignment in the demodulation and correction of seeing-induced crosstalk.

2. Was there something different in the reduction of Stokes $V$ and for $Q$ or $U$ ? One can try to obtain $V$ as $Q$ or $U$, in other words, to compute $V$ from three different modulations. In this case the test was done with $V=\frac{1}{2}(I+$ $Q)+\frac{1}{2}(I+U)-(I-V)$. The resulting Stokes $V$ exhibits the same net signal.

3. During the observing day other three scans where performed. The one from the same region as the scan under study has the same imbalanced flux, while the other two have different net fluxes, negative and positive. This fact dismisses the idea of systematic mistakes in the data reduction or in the instrument that could generate the same kind of signal.

4. Is this effect due to flat fielding? One set of flat field images was reduced as it were data. The result was no Stokes $V$ signal at all, thus no net $V$ signal. 


\subsection{PCA classification}

From here until the end of this Chapter a set of Stokes $V$ profiles clearly above noise is selected from the data. A threshold of three times the noise level was chosen in order to separate clear polarization signals from very noisy profiles. Pixels with profiles whose peak of polarization is above the threshold in both IR and visible data correspond to $40 \%$ of the FOV. This data set includes some 2350 pixels.

The profiles in the selected data set were classified using a principal component analysis algorithm (PCA, e.g., Everitt 1995). This classification was done in a similar way as by Sánchez Almeida \& Lites (2000, Section 3.2). The aim is, on the one hand, to have a reduced number of representative profiles to make a first study and, on the other hand, to have very clear profiles to perform a first inversion (either ME in Section 4.5 or MISMA in Section 4.6) and to use the obtained parameters as the initialization for inversion of individual profiles. The classification is done simultaneously for visible and IR Stokes $V$ profiles. The profiles of the four lines were scaled to the blue lobe of the Fe I $\lambda 6302.5 \AA$ Stokes $V$ profile. The PCA analysis gave 38 classes. This huge number of classes compared with that obtained for visible lines by Sánchez Almeida \& Lites (2000) or for IR lines by Khomenko et al. (2003) is due to the classification of a combination of the different asymmetries found in the Stokes $V$ profiles of the two pairs of lines.

Some of these classes are shown in Figs. 4.6 and 4.7 (circles are the observed classes). Note how IR profiles from classes 0 and 4 have similar tails of polarization (extending far out from their extreme peaks) as those expected in Fig. 2.6. Such profiles are similar to class 0 in Khomenko et al. (2003, Fig. 1). The first 6 classes include $42 \%$ of the total number of profiles. One conspicuous fact is that some classes (e.g., 6, 20) show opposite polarity in the visible and in the IR lines. Such a circumstance was expected from the magnetogram in Fig. 4.2. Approximately $20 \%$ of the profiles show this effect. The classification was done in a way that all profiles with large polarization signals $\left(1 \% I_{c}\right.$ in visible and $0.7 \% I_{c}$ in IR) were included in one single class in order not to contaminate the other classes. These network profiles are in class 10, and they show characteristic broad IR profiles (also observed in plage regions by Stenflo et al. 1987). 


\subsection{Milne-Eddington inversion}

In order to estimate the magnetic field strength a Milne-Eddington inversion was performed. This is a simple technique, but it can be considered as a first approximation to compare with other results in the literature.

One of the simplest solutions of the radiative transfer equation for polarized radiation (RTE, Eq. 2.6) is the solution for an atmosphere under the MilneEddington (ME) approximation. This simplification yields an analytical solution to the RTE. An inversion code based on such an atmospheric model is applied to the data and gives a fast estimation of the magnetic field strength.

The Milne-Eddington model works with five approximations:

1. The magnetic field vector is constant, i.e., independent of height or optical depth $\tau$.

2. All parameters related with the profile of the absorption coefficient (Doppler broadening $\Delta \lambda_{D}$ and damping constant $a$ ) are independent of optical depth.

3. There are no velocity fields.

4. The ratio $\eta_{0}$ between line and continuum absorption coefficients is constant with $\tau$.

5. The Planck function depends linearly on $\tau$ : $B_{T}(\tau)=B_{0}+B_{1} \tau$.

One can obtain an analytical solution for the Stokes parameters from the above simplifications. The vectorial form of the Eq. 2.6 can be written as

$$
\frac{d \mathbf{I}}{d \tau}=-\mathbf{K}(\mathbf{I}-\mathbf{S})
$$

where $\mathbf{K}$ is the absorption matrix, $\mathbf{I}=(I, Q, U, V)^{T}$ is the Stokes vector, and $\mathbf{S}=$ $\left(B_{T}, 0,0,0\right)^{T}$ the LTE source vector. From the above hypotheses the absorption matrix is constant with $\tau$, and from the linear dependence of the Planck function with $\tau\left(B_{T}(\tau)=B_{0}+B_{1} \tau\right)$ one obtains the solution for the emergent Stokes parameters (more details in Landi Degl'Innocenti 1992)

$$
\begin{aligned}
I & =B_{0}+B_{1} \Delta^{-1}\left[\eta_{I}\left(\eta_{I}^{2}+\rho_{Q}^{2}+\rho_{U}^{2}+\rho_{V}^{2}\right)\right] \\
Q & =-B_{1} \Delta^{-1}\left[\eta_{I}^{2} \eta_{Q}+\eta_{I}\left(\eta_{V} \rho_{U}-\eta_{U} \rho_{V}\right)+\rho_{Q}\left(\eta_{Q} \rho_{Q}+\eta_{U} \rho_{U}+\eta_{V} \rho_{V}\right)\right] \\
U & =-B_{1} \Delta^{-1}\left[\eta_{I}^{2} \eta_{U}+\eta_{I}\left(\eta_{Q} \rho_{V}-\eta_{V} \rho_{Q}\right)+\rho_{Q}\left(\eta_{Q} \rho_{Q}+\eta_{U} \rho_{U}+\eta_{V} \rho_{V}\right)\right] \\
V & =-B_{1} \Delta^{-1}\left[\eta_{I}^{2} \eta_{V}+\eta_{I}\left(\eta_{U} \rho_{Q}-\eta_{Q} \rho_{U}\right)+\rho_{Q}\left(\eta_{Q} \rho_{Q}+\eta_{U} \rho_{U}+\eta_{V} \rho_{V}\right)\right]
\end{aligned}
$$

with $\Delta=\eta_{I}^{2}\left[\eta_{I}^{2}+\eta_{U}^{2}+\eta_{U}^{2}+\eta_{V}^{2}+\rho_{Q}^{2}+\rho_{U}^{2}+\rho_{V}^{2}\right]-\left(\eta_{Q} \rho_{Q}+\eta_{U} \rho_{U}+\eta_{V} \rho_{V}\right)^{2}$. This solution is generally referred to the Unno-Rachkovsky solution (see Unno 1956). 


\begin{tabular}{lcl}
\cline { 2 - 3 } Table 4.4: Free parameters & $B$ & Magnetic field strength \\
which can be modified by MILK & $\theta$ & Magnetic field inclination \\
to fit the Stokes profiles of the & $\chi$ & Magnetic field azimuth \\
lines. & $\eta_{0}$ & Line strength \\
$\Delta \lambda_{D}$ & Doppler width \\
$a$ & Damping parameter \\
& $v_{D}$ & Doppler shift \\
$B_{0}$ & Constant part of Planck function \\
$B_{1}$ & Slope of Planck function \\
$\xi$ & Macroturbulence \\
$f_{s l}$ & Fraction of stray light \\
\hline
\end{tabular}

The inversion code used was MILK, which is part of the Community Inversion Codes $^{4}$ developed at the High Altitude Observatory (see Socas-Navarro 2001). The code adjusts 11 different atmospheric and magnetic field parameters in order to fit the observed profiles (for a description of the procedure, see Skumanich \& Lites 1987). Table 4.4 characterizes all parameters.

The code assumes an atmosphere with a single magnetic component. Since the magnetic fields occupy only a small fraction of the quiet Sun, the stray light, or false light, is considered to be the non-magnetic component and the filling factor of the magnetic component is then

$$
\alpha=1-f_{s l}
$$

The expected $\alpha$ in IN fields is of the order of a few percent (see Section 3.4.2; Sánchez Almeida \& Lites 2000; Khomenko et al. 2003). Thus, the stray light was introduced in the code as the Stokes $I$ profile from each inverted class or pixel (there is almost no influence from the magnetic component).

The inversion was performed separately for the two pairs of lines, and was done for the Stokes $I$ and Stokes $V$ profiles from each class or individual pixel. Here, the only parameters of relevance from the inversion are the magnetic field strength $(B)$, the filling factor $(\alpha)$ and the Doppler velocity measured from the Doppler shift. Since only Stokes $V$ profiles are used for the determination of the field strength, the latter is, in the weak field approximation, the component along the line of sight. However, this value is very close to the total field strength because magnetic field vectors in the quiet Sun are mainly vertical.

Figures 4.6 and 4.7 show the result of the inversion for 12 classes. The observed classes (circles) are the average of the individual Stokes $V$ profiles which belong to each class, and the ME fits are also displayed (solid line). The average of Stokes I profiles from each class was also used in the inversion. In these figures

\footnotetext{
${ }^{4}$ See http://www.hao.ucar.edu/public/research/cic/
} 

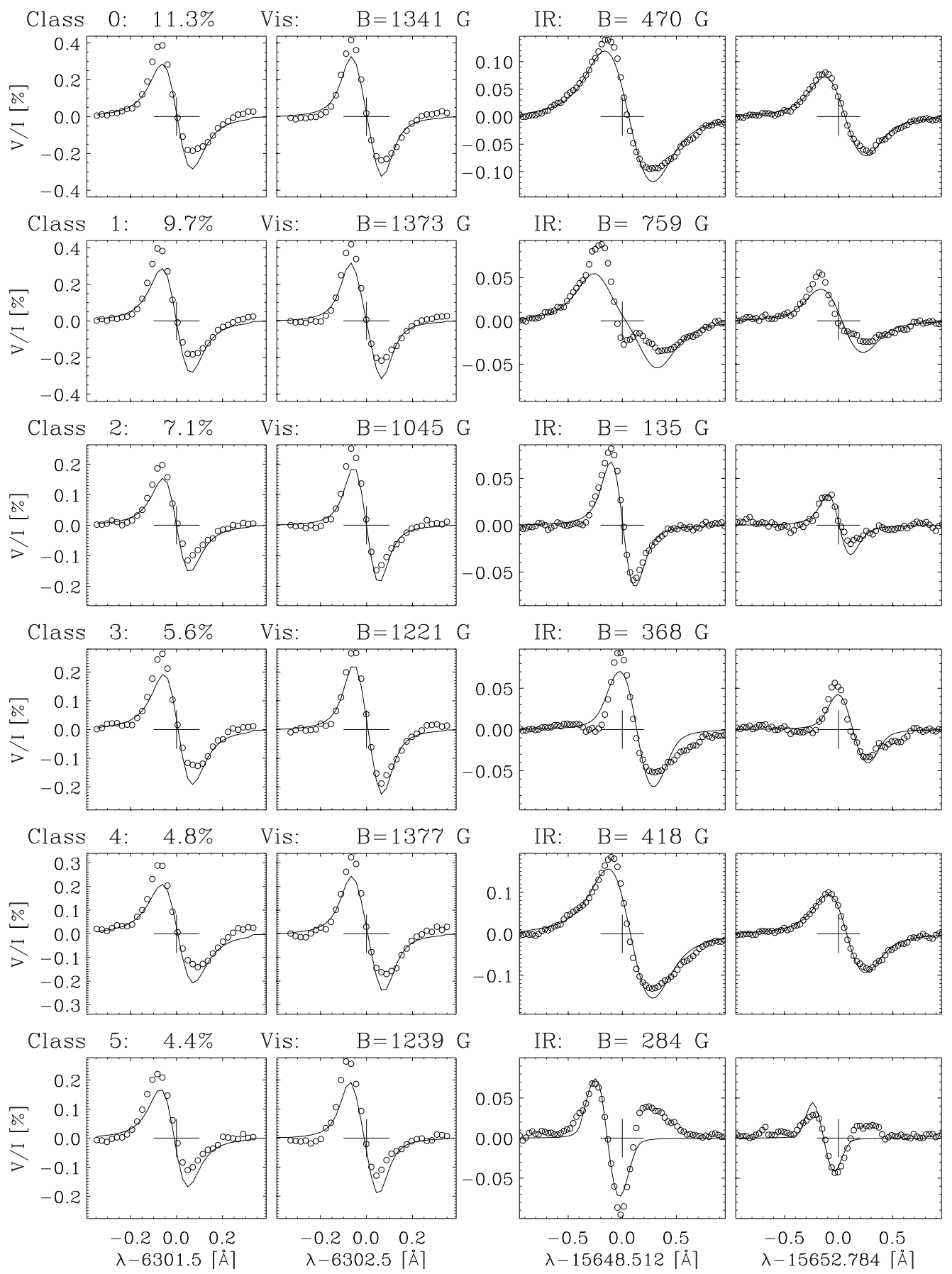

Figure 4.6: Stokes $V$ profiles from the 6 most abundant classes. The circles represent the observed mean profiles of Fe I $\lambda 6301.5 \AA$, Fe I $\lambda 6302.5 \AA$, Fe I $\lambda 15648 \AA$ and Fe I $\lambda 15652 \AA$ (from left to right). The solid lines show the ME fit. 

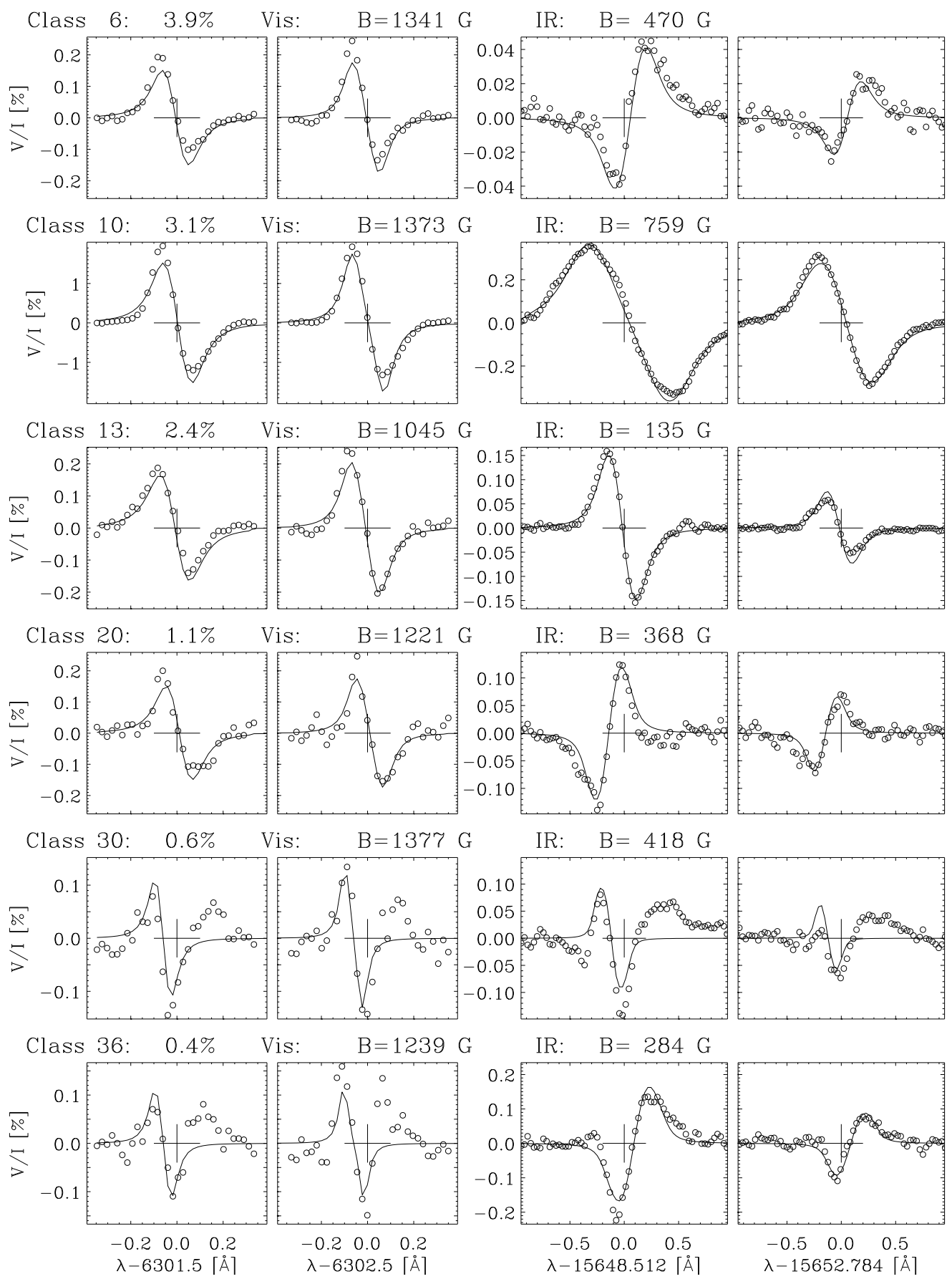

Figure 4.7: Same as in Fig. 4.6 for other 6 characteristic classes. Note that classes 6 and 20 have opposite polarities in the visible and IR lines, class 10 comes from network profiles, and classes 30 and 36 have three lobes in visible. 
the level of polarization is given in percentage of the continuum of Stokes $I$. The spectral range used to display the visible profiles is $0.8 \AA$, while the IR profiles need $2 \AA$. Since the ME inversion uses only one magnetic component in a very simple atmosphere there is no possibility to fit the asymmetries which are clearly present in all classes. Sometimes, the $V$ profiles in the IR are very broad (e.g., class $0,1,4,10$ ). Here, the ME inversion finds, in order to fit the observations, an unrealistically large macroturbulence velocity parameter. Very likely, the broad profiles result from the presence of both weak and strong magnetic fields (see Section 2.3), or at least from magnetic field gradients. However, that effect does not affect the estimation of the magnetic field.

The magnetic field strength computed for the visible and the IR are also given in Fig. 4.6 and 4.7. In most of the 38 classes the magnetic field is above $1 \mathrm{kG}$ from inversions in the visible and below $500 \mathrm{G}$ from inversions in the IR. Such a behavior was expected from previous works (see Section 2.3). One can say that the ME code estimates the magnetic field in the visible by the ratio between the lobes of the Stokes $V$ profiles from Fe I $\lambda 6302.5 \AA$ and Fe I $\lambda 6301.5 \AA$ (similar to the ratio method presented in Section 3.4.2). A ratio close to 1 means a magnetic field above $1 \mathrm{kG}$, while a ratio of 0.6 is expected for weak fields (see Section 5 in Socas-Navarro \& Sánchez Almeida 2002). Since the sensitivity to the magnetic field, which is proportional to $\Delta \lambda_{B} / \Delta \lambda_{D} \propto g \lambda B$, is approximately three times larger for Fe I $\lambda 15648 \AA$, MILK estimates the magnetic field strength in the IR essentially from the separation of the lobes. Khomenko et al. (2003) computed the field strength using only the separation of the lobes, finding that the minimum reliable intrinsic field measured with this method was $300 \mathrm{G}$. Weaker fields produce profiles with similar splitting ${ }^{5}$, thus the field strength and the filling factor cannot be determined separately. Since the ME inversion takes into account also the ratio between maxima of the lobes in the Stokes $V$ profiles of Fe I $\lambda 15648 \AA$ and Fe I $\lambda 15652 \AA$, the lowest limit of field strength with this technique turned out to be smaller. There are classes with intrinsic fields lower than $150 \mathrm{G}$ (e.g., class 2 and 13).

At first sight the difference of the estimation of the magnetic field strength from the visible and IR lines is surprising. The estimation in the visible can be spoiled by noise in the Stokes $V$ profiles (see Bellot Rubio \& Collados 2003). However, the observed classes possess almost no noise, since they represent averages of many individual profiles. Thus the inversion can not be affected by noise. An inspection of the maxima of the lobes in the two visible lines results in ratios between 0.75 and 1 in most of the classes, which correspond to $\mathrm{kG}$ fields.

\footnotetext{
${ }^{5}$ The saturation of Fe I $\lambda 15648 \AA$ occurs at fields of $300-400$ G. See Section 2.2 .
} 

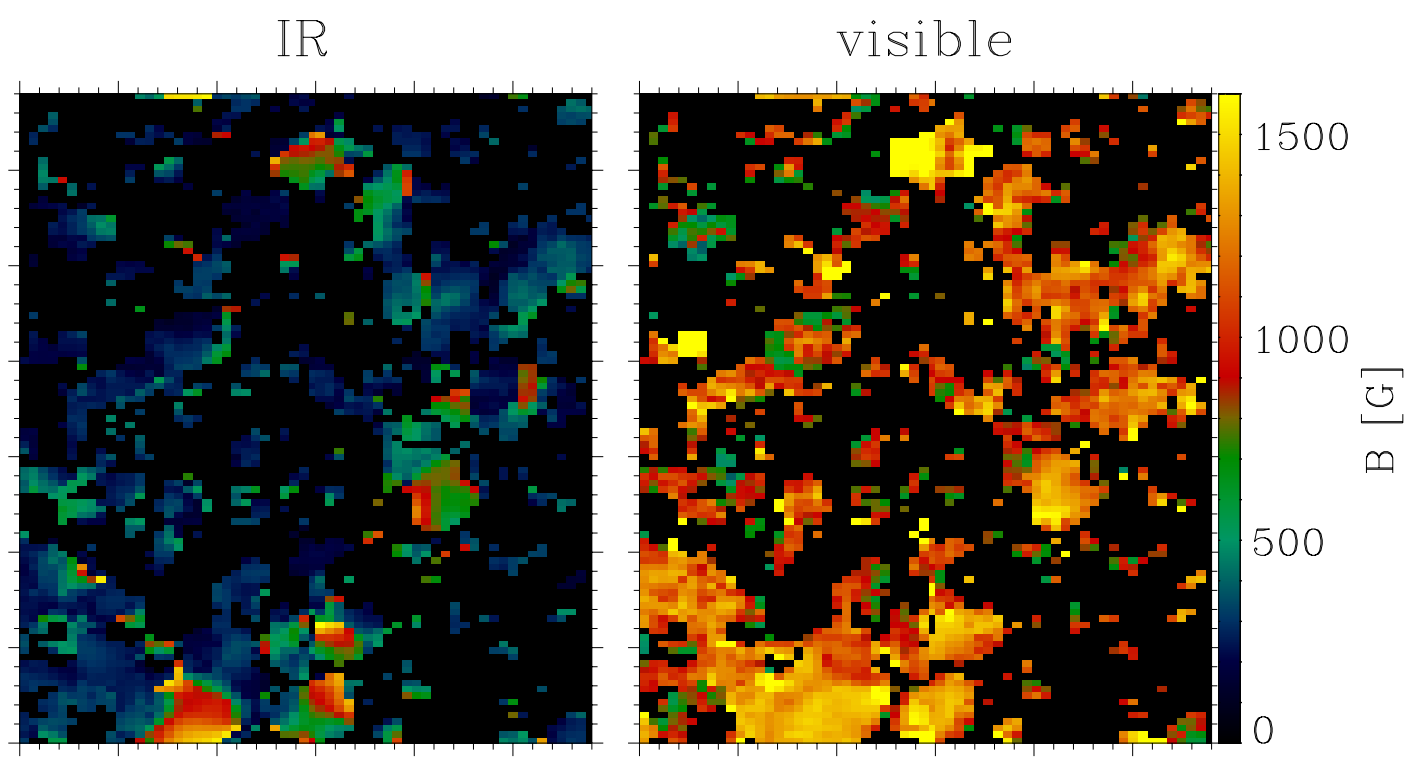

Figure 4.8: Maps of the magnetic field strength obtained from ME inversion for the IR (left) and the visible (right) lines. Separation between tickmarks in arcsec. Both maps are equally scaled, and black represents pixels with signals too low to be analyzed.

\subsubsection{Magnetic field strength}

The parameters from the inversion of classes were used as initialization of the inversion of the individual profiles corresponding to each class. Figure 4.8 shows maps of the magnetic field strength resulting from the inversion of individual pixels for the IR inversion (left) and the visible inversion (right). Both maps have the same scale. As it was observed in the inversion of classes, most of the pixels have sub-kG magnetic fields inferred from IR lines while they have $\mathrm{kG}$ fields from visible inversion. The visible magnetic field map resembles that obtained by Socas-Navarro \& Sánchez Almeida (2002, Fig. 5) for the same pair of iron lines. However there are some differences. The pixels analyzed here cover $40 \%$ of the FOV (compared with the $27 \%$ in their work) and there is almost no network (only $3 \%$ of the FOV, versus their $7 \%$ ). Strong fields are present everywhere, and the highest values correspond to the network regions. The few weak fields are located in the external pixels of the magnetic patches. The map from IR data shows some strong fields apart from the network region. In general, the patches exhibit in the center slightly stronger fields than at the borders. This effect was also observed by Khomenko et al. (2003). 

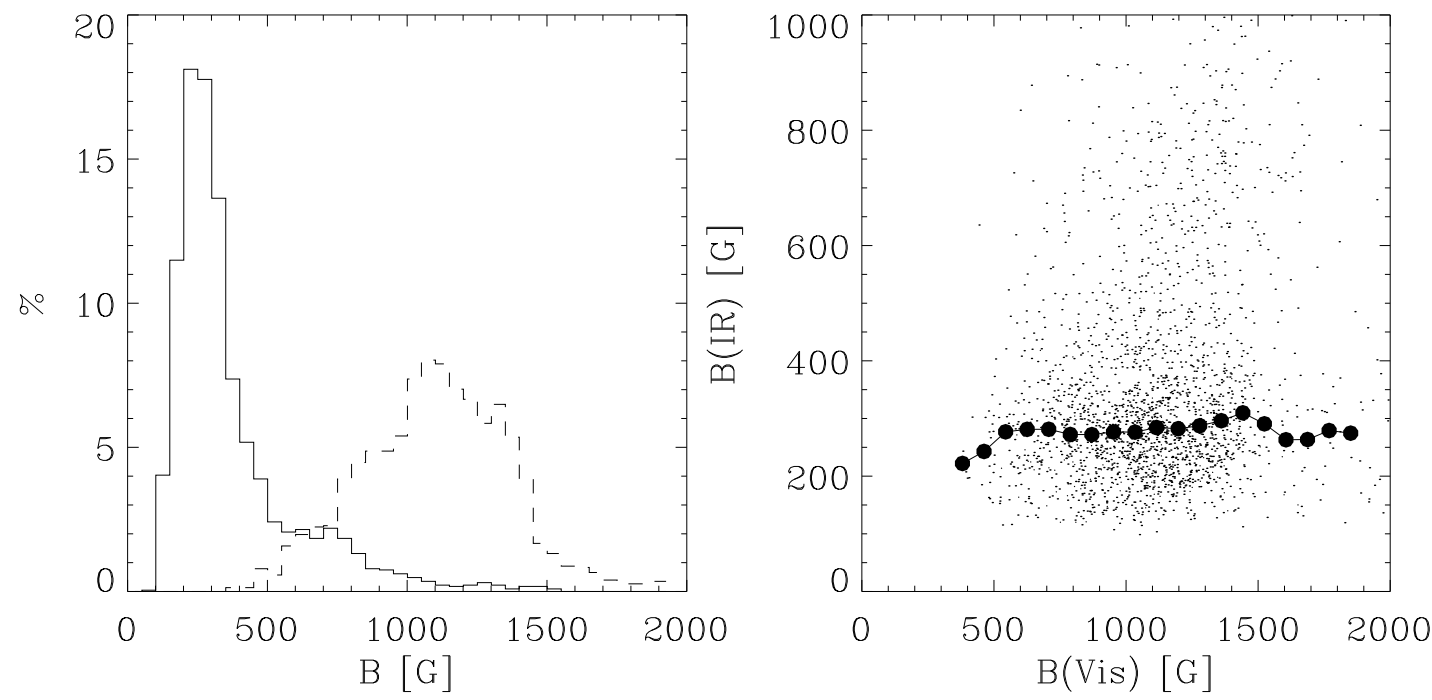

Figure 4.9: Comparison between results from ME inversion for visible and IR lines. Left: Histograms of $B$ (magnetic field strength) for IR (solid line) and for the visible (dashed). Right: B for IR vs. B for visible. Filled circles are averages over $\mathrm{B}(\mathrm{IR})$ for equally spaced bins in $\mathrm{B}(\mathrm{Vis})$.

Figure 4.9 shows a histogram (left panel) of the occurrence of the magnetic field strength in the visible (dashed) and IR (solid). On the one hand, the histogram for IR observations is clearly concentrated in weak fields and it is quite similar to that obtained by Khomenko et. al (2003, Fig. 6) for the same lines. On the other hand, the histogram for the visible is concentrated in $\mathrm{kG}$ fields. The average magnetic field strength was $400 \mathrm{G}$ in the IR and $1100 \mathrm{G}$ in the visible.

The scatter plot (Fig. 4.9 right) of the magnetic field strength obtained from the IR $(B(\mathrm{IR})) v s$. the ones obtained from the visible $(B$ (Vis)) shows no dependence, which means that both magnetic fields are independent. The filled circles are averages of $B(\mathrm{IR})$ for bins of $B$ (Vis), neglecting those pixels with strong fields in the IR. This behaviour has to be ascribed to the property of the quiet Sun harboring two magnetic components, one with weak fields and another with strong fields. Due to the different sensitivity of the lines the polarization signal of the IR Stokes $V$ profiles are mainly produced by the weak component while the signals of visible profiles are produced mainly by strong signals (see Fig. 2.4). This is a strong argument showing that in the quiet Sun the $\mathrm{kG}$ magnetic fields co-exist with sub-kG fields. It must be kept in mind that this result on co-spatiality refers to spatial resolution elements of 1 .".5.

Figure 4.10 displays the maps for the filling factor (top panels) and the magnetic flux (bottom panels). The latter was defined as the magnetic field strength times 
IR
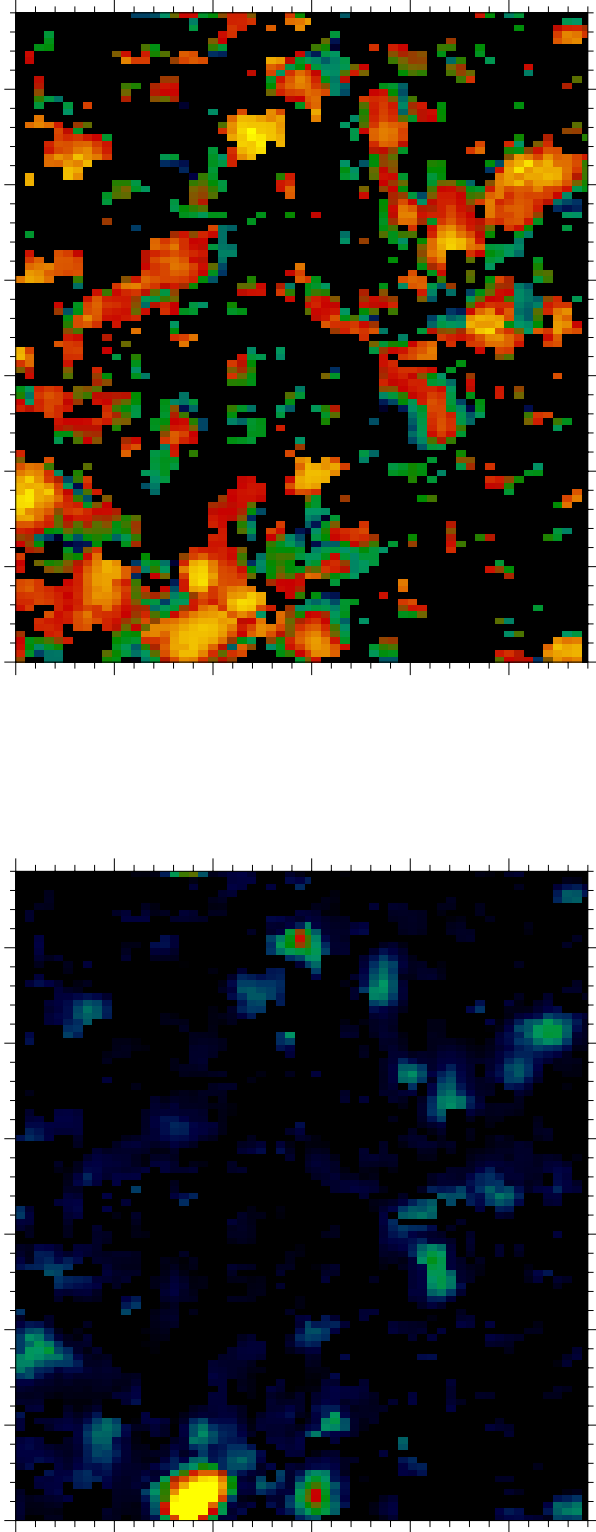

visible

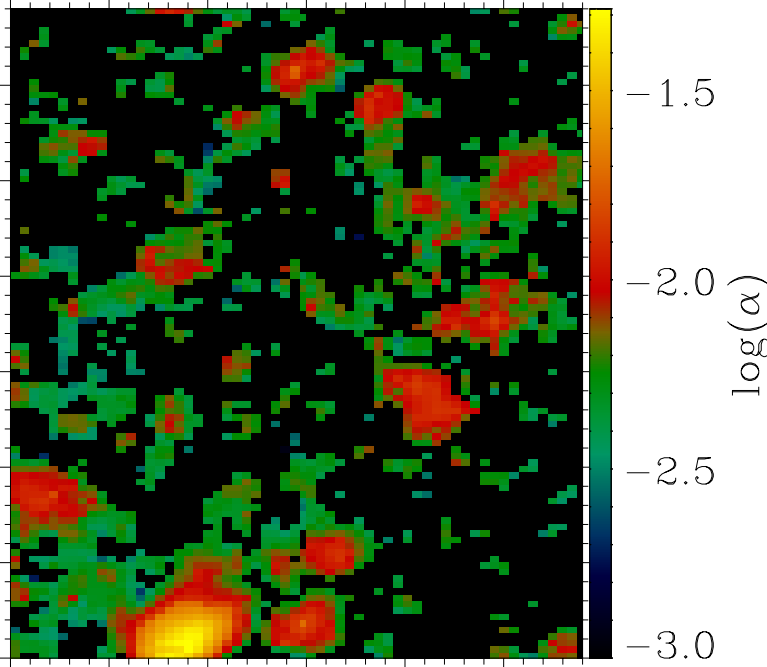

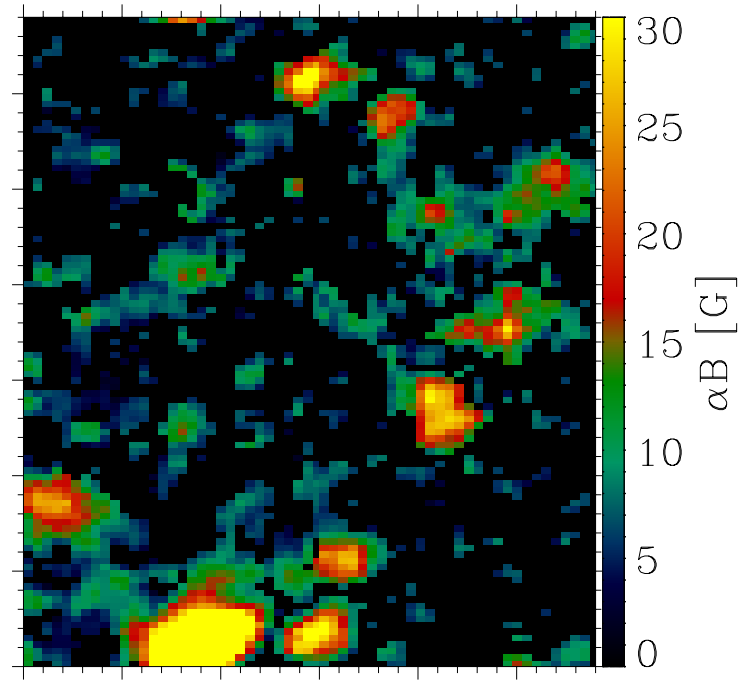

Figure 4.10: Maps of the filling factor in logarithmic scale ( $\log \alpha$, top panels) and of the magnetic flux density ( $\alpha B$, bottom) obtained from ME inversion in IR (left) and visible (right). Separation between tickmarks in arcsec. Both pairs of maps are equally scaled, and black is at pixels with too low polarimetric signals. 
the filling factor $(\alpha B)$. An inspection shows that the filling factors are smaller in the visible than in the IR. Some pixels in the visible have $\alpha$ larger than $1 \%(\log$ $\alpha=-2$ ) while more magnetic fields in IR pixels have larger magnetic occupation than 1\%. The filling factors obtained in Socas-Navarro \& Sánchez Almeida (2002) for the visible lines have larger values than in this work. The difference is due to the better spatial resolution of their observations ${ }^{6}$. A comparison with the results of Khomenko et al. (2003) shows that the same reduction of the filling factors occurs in the IR analysis.

In contrast to this, the maps of flux density (bottom panels in Fig. 4.10) indicate that the magnetic flux is larger when it is inferred from the visible lines than from the IR lines. This effect was already observed in the magnetograms obtained from less sophisticated techniques (see Section. 4.3). These magnetograms are similar to the maps of magnetic flux from ME inversion, with the exception that magnetic flux in the visible turns out to be larger when it is calculated with the ME inversion.

The plot of the field strength as a function of the magnetic flux density (Fig. $4.11 \mathrm{a}$ and $4.11 \mathrm{~b}$ ) shows a clear dependence in both data sets. The larger the intrinsic field the more flux is detected. A similar result was already reported from plages and network (Stenflo \& Harvey 1985) and from internetwork in IR lines (Lin 1995; Khomenko et al. 2003) and in the visible lines (Grossmann-Doerth et al. 1996; Socas-Navarro \& Sánchez Almeida 2002). Sánchez Almeida \& Lites (2000) ascribed this effect to the dependence of the transparency of the atmosphere with the magnetic flux. They propose that magnetic structures with larger flux are more transparent than those with smaller flux. Since the magnetic field strength is estimated from a small range of opacities, the Stokes $V$ profile from a more transparent structure (with larger flux) is produced by plasma in lower layers. The magnetic field strength decreases with height, hence more transparent structures are associated with larger intrinsic fields.

From the classification one can divide the profiles into three main categories. The first one corresponds to those pixels with ordinary Stokes $V$ profiles (called hereafter w1 profiles). They are those profiles with two lobes in all four spectral lines, and the polarity of the magnetic fields is the same in visible and IR (e.g., classes $0,1,2$ ). This is the most abundant group (for this reason profiles are named ordinary) with an occurrence of 59\%. The second category (w2) stands for those profiles with two lobes in all four lines, but whose polarity is different in the visible and IR profiles (e.g., classes 6, 20). $20 \%$ of the analyzed pixels have profiles of this kind. The third group (w3) contains profiles with three or more lobes in at least one pair of lines. These profiles need magnetic fields with mixed polarities in order to fit such an asymmetry. Approximately $17 \%$ of the pixels

6 Their resolution was $1^{\prime \prime}$, thus their resolution element is $2-3$ times smaller compared with the one in this work. 
Table 4.5: Average magnetic field strength $(B)$, filling factor $(\alpha)$ and magnetic flux density $(\alpha B)$ from IR and visible inversions. The different categories correspond to ordinary profiles (w1, see text), opposite polarity profiles (w2), three-lobes profiles (w3), and network profiles (w4). The values are also given for profiles apart from network profiles (all-w4). Note that $B, \alpha$, and $\alpha B$ are average values by themselves, such that $B \times \alpha=\alpha B$ must not necessarily hold.

\begin{tabular}{cc|ccc|ccc}
\hline \hline & & \multicolumn{3}{|c|}{ IR } & \multicolumn{3}{c}{ Visible } \\
\hline Group & $\begin{array}{c}\text { Occurrence } \\
{[\%]}\end{array}$ & $B$ & $\alpha$ & $\alpha B$ & $B$ & $\alpha$ & $\alpha B$ \\
& {$[\mathrm{G}]$} & {$[\%]$} & {$[\mathrm{G}]$} & {$[\mathrm{G}]$} & {$[\%]$} & {$[\mathrm{G}]$} \\
\hline all & 100 & 390 & 1.14 & 4.0 & 1120 & 1.06 & 12.3 \\
w1 & 59 & 400 & 1.3 & 4.0 & 1150 & 1.02 & 11.8 \\
w2 & 20 & 350 & 0.8 & 2.5 & 1040 & 0.7 & 7.6 \\
w3 & 17 & 290 & 0.9 & 2.5 & 1060 & 0.7 & 7.1 \\
w4 & 3 & 900 & 2.1 & 19.6 & 1380 & 5.5 & 78 \\
all-w4 & 97 & 370 & 1.1 & 3.4 & 1110 & 0.9 & 10 \\
\hline \hline
\end{tabular}

belong to this group. Most of the classes of this kind show three lobes in the IR lines exclusively (e.g., class 5 ). Only $2 \%$ of the profiles have three lobes in the visible (e.g., classes 30,36). Sánchez Almeida \& Lites (2000) observed $6 \%$ of profiles with three lobes in the same visible lines, while Khomenko et al. (2003) classified $35 \%$ of the pixels with such a quality. Finally, the presence of Stokes $V$ profiles with 4 lobes in the IR lines is noteworthy (e.g., class 30), they occur in $3 \%$ of the pixels. A fourth category was assigned to the profiles belonging to network areas (w4, class 10). Only $3 \%$ of all pixels contain these profiles with strong signals. It is important to remark the problem of fitting w3 profiles with a one-component model. The analysis misses part of the magnetic field since the inversion code tries to fit two of the three lobes with one magnetic field and one velocity. Yet, w2 profiles must be the result of mixed polarities. It is however not evident to find atmospheric structures and dynamics able to reproduce such profiles.

Table 4.5 presents the average values of intrinsic fields, filling factors and flux densities over the pixels belonging to either one of the categories defined above and computed by inversion of visible and IR lines. It is remarkable that the group w1 presents higher filling factors and fluxes in both spectral ranges than the groups w2 and w3. In general profiles with three lobes and with reversed polarity have lower polarization signals. It is conspicuous that these groups have less signal in both visible and IR profiles. Such a behavior was expected from the observation that the number of profiles with mixed polarities increases with decreasing polarization signal (Sánchez Almeida \& Lites 2000). There is a slight 

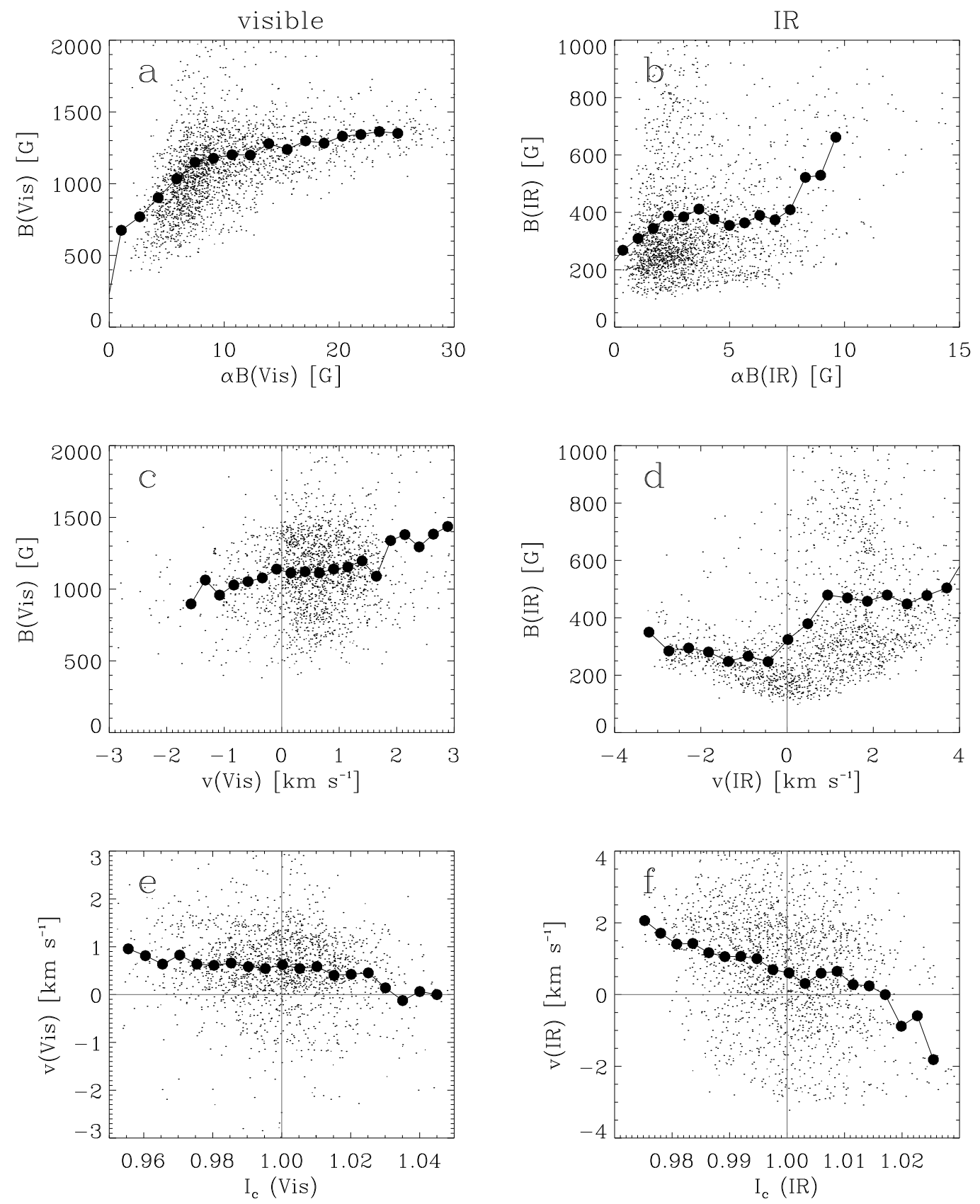

Figure 4.11: Magnetic field strength as a function of the flux density $\alpha B$ (a and b), magnetic field strength $v s$. velocity (c and d) and velocity vs. the continuum intensity (e and f) for visible lines (left panels) and IR lines (right panels). Filled circles are averages over the ordinate within bins of the abscissa, as in Fig. 4.9. 
reduction in the intrinsic fields from w1 profiles to w2 and w3. The diminishing occurs in both the visible and IR determinations and it can be explained with the above mentioned dependence of the field strength on the flux (and consequently on the filling factor). Since the w4 group corresponds to network it shows strong fields in the visible and IR, and one can see that there is a small influence of this group on the average values from all profiles (comparing with all-w4). The important result is to be emphasized that the fluxes obtained from inversions in IR and visible in the network, differ by a factor of 4 . From now, and to avoid any contamination in the study of IN fields, all calculations will avoid group w4.

\subsubsection{Velocities}

Some interesting results can be obtained from the line-of-sight velocity computed by the ME code. This velocity is very similar to that obtained from the zero crossing of the Stokes $V$ profiles and deviations are due to asymmetries of the profiles. However, the study done for the velocities obtained in both ways shows that the essential properties are the same.

Figure 4.12 displays a histogram of the velocities (left). The first and surprising
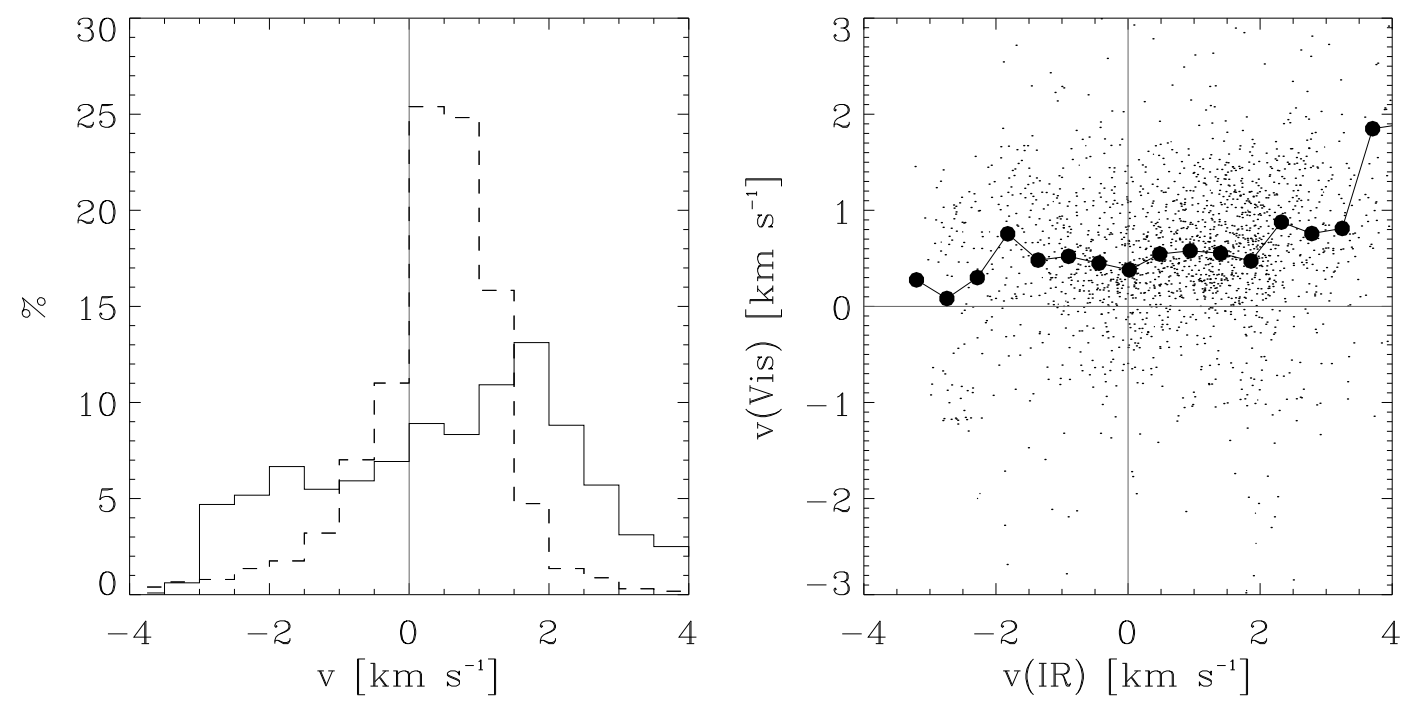

Figure 4.12: Comparison between results from ME inversion for visible and IR lines. Left: Histograms for velocity computed from IR lines (solid line) and from visible lines (dashed). Right: velocity for visible vs. velocity for IR. Positive velocities correspond to downflows in the solar atmosphere. Filled circles are averages over the ordinate within bins of the abscissa as in right panel of Fig. 4.9. 
Table 4.6: Average velocities from inversion of IR and visible observations. Groups are defined as in Table 4.5.

\begin{tabular}{ccc}
\hline \hline Group & $\mathrm{v}(\mathrm{IR})\left[\mathrm{km} \mathrm{s}^{-1}\right]$ & $\mathrm{v}(\mathrm{Vis})\left[\mathrm{km} \mathrm{s}^{-1}\right]$ \\
\hline all-w4 & $+0.6 \pm 1.8$ & $+0.36 \pm 1.0$ \\
$\mathrm{w} 1$ & $+0.9 \pm 1.5$ & $+0.6 \pm 0.8$ \\
$\mathrm{w} 2$ & $+0.35 \pm 1.7$ & $+0.4 \pm 0.9$ \\
w3 & $-0.15 \pm 2.6$ & $-0.6 \pm 1.2$ \\
\hline \hline
\end{tabular}

phenomenon is the difference in the width of the histograms. The distribution of IR velocities (solid line) is much more spread than the distribution of visible velocities (dashed line). The average velocity was $600 \mathrm{~m} \mathrm{~s}^{-1}$ for the IR data and $360 \mathrm{~m} \mathrm{~s}^{-1}$ for the visible data. Similar net downflows were also observed in the IR (550 $\mathrm{m} \mathrm{s}^{-1}$ in Khomenko et al. 2003). The dispersion of the velocities amounts to $1 \mathrm{~km} \mathrm{~s}^{-1}$ in the visible and $1.8 \mathrm{~km} \mathrm{~s}^{-1}$ in the IR. Such a difference can be only explained if the two Stokes $V$ profiles come from different magnetic components. Many ingredients may produce this difference in the dispersion, e.g., a difference in the height of formation of the Stokes $V$ profiles. The discrepancy of these velocities is an open question.

Table 4.6 presents the average velocities for the different categories defined in Section 4.5.1. It is conspicuous that w1 exhibits a larger net downflow and a smaller dispersion than other groups. Velocities in group w3 have a huge dispersion due to the kind of profiles inverted. These velocities are not very reliable since the profiles have three lobes and the ME code fits two of them, thus there is always a large velocity (see class 5 in Fig. 4.6). As a consequence, w3 profiles may be contaminating the velocity calculations, and the actual dispersion is slightly smaller. Thus from this point on, the calculations of velocities will neglect the group w3.

A scatter plot of the velocity computed from visible profiles as a function of the velocity from IR profiles is given in the right panel of Fig. 4.12. In addition to the above mentioned larger dispersion of velocities, there is a weak dependence of $\mathrm{v}$ (Vis) on $\mathrm{v}(\mathrm{IR})$. The arguments given before pointed out the independence of the magnetic components deduced from each visible and IR pair of lines. Hence, the velocities should also be independent and the cause of the small correlation can be the 5-min oscillation with Doppler velocities of few hundred $\mathrm{m} \mathrm{s}^{-1}$.

Figures $4.11 \mathrm{c}$ and $\mathrm{d}$ show the field strength as a function of the velocity. There is an increase of the intrinsic field for increasing downflows in the visible and IR. However, the IR fields show a perceptible increase for larger upflows with no clear explanation.

When the velocity is plotted as a function of the continuum intensity (Figs. $4.11 \mathrm{e}$ and $\mathrm{f}$ ) the tendency is that downflows are concentrated in intergranular lanes. However, the resolution is not good enough to make a deep analysis differentiating between granule and intergranule. 


\subsubsection{Probability Density Function (PDF)}

From the magnetic field and filling factor procured by the ME inversion, the probability density function (PDF) of the magnetic field strength has been computed. The PDF gives the probability to find a specific magnetic field strength in the observed IN region. One of the main results discussed in the above sections is the fact that visible and IR lines are tracing different magnetic structures. Under this assumption the PDF was computed as the sum of the PDF for the visible inversion and the PDF for IR inversion. A range of magnetic fields from 0 to $2000 \mathrm{G}$ was divided into bins of $50 \mathrm{G}$ width. Each individual intrinsic field $B$ is assigned to one bin. The probability to find the magnetic field of one bin is the sum of the filling factors of all pixels with $B$ belonging to this bin. Finally, the normalization of the PDF takes into account the pixels not under analysis due to low polarization signal. Thus, $60 \%$ of the FOV are assumed to be field-free.

Figure 4.13 shows the total PDF (solid line) on a logarithmic scale. The main characteristic is that weaker fields have a larger probability than strong fields. In other words, weaker fields occupy more of the FOV. This was already clear from the maps of the filling factors (Fig. 4.10). There it is seen that the magnetic fields determined from the IR lines, which trace mainly weak fields, have larger occupation fractions than the magnetic fields from visible lines. Figure 4.13 also includes separately the PDF from the IR data (dashed line) and from the visible data (dotted line).

Table 4.7 exhibits the other main peculiarity. It presents the filling factor, magnetic flux density and fraction of magnetic flux for different parts of the PDF. The average flux density is $5.1 \mathrm{G}$ filling just $0.81 \%$ of the FOV. The part of the PDF representing the visible lines exhibits a smaller filling factor but shows a larger amount of flux than the IR PDF. The magnetic flux for field strengths larger than $500 \mathrm{G}$ was $81 \%$ of the total flux, which is in agreement with the PDF suggested by Socas-Navarro \& Sánchez Almeida (2003) plotted in Fig. 2.5.

This excess of $\mathrm{kG}$ flux together with the larger filling factor for weak fields are the necessary ingredients to solve the controversy presented in Section 2.3. However, one must have in mind the simplicity of the techniques used in this section. More complicated models and inversions are required to reproduce the asymmetries and obtain a more reliable magnetic field distribution than from a one-component model. The advantage of using such a simple scenario is that the results obtained are less affected by noise and less depending on initial conditions, since the inversions need only a few free parameters. 


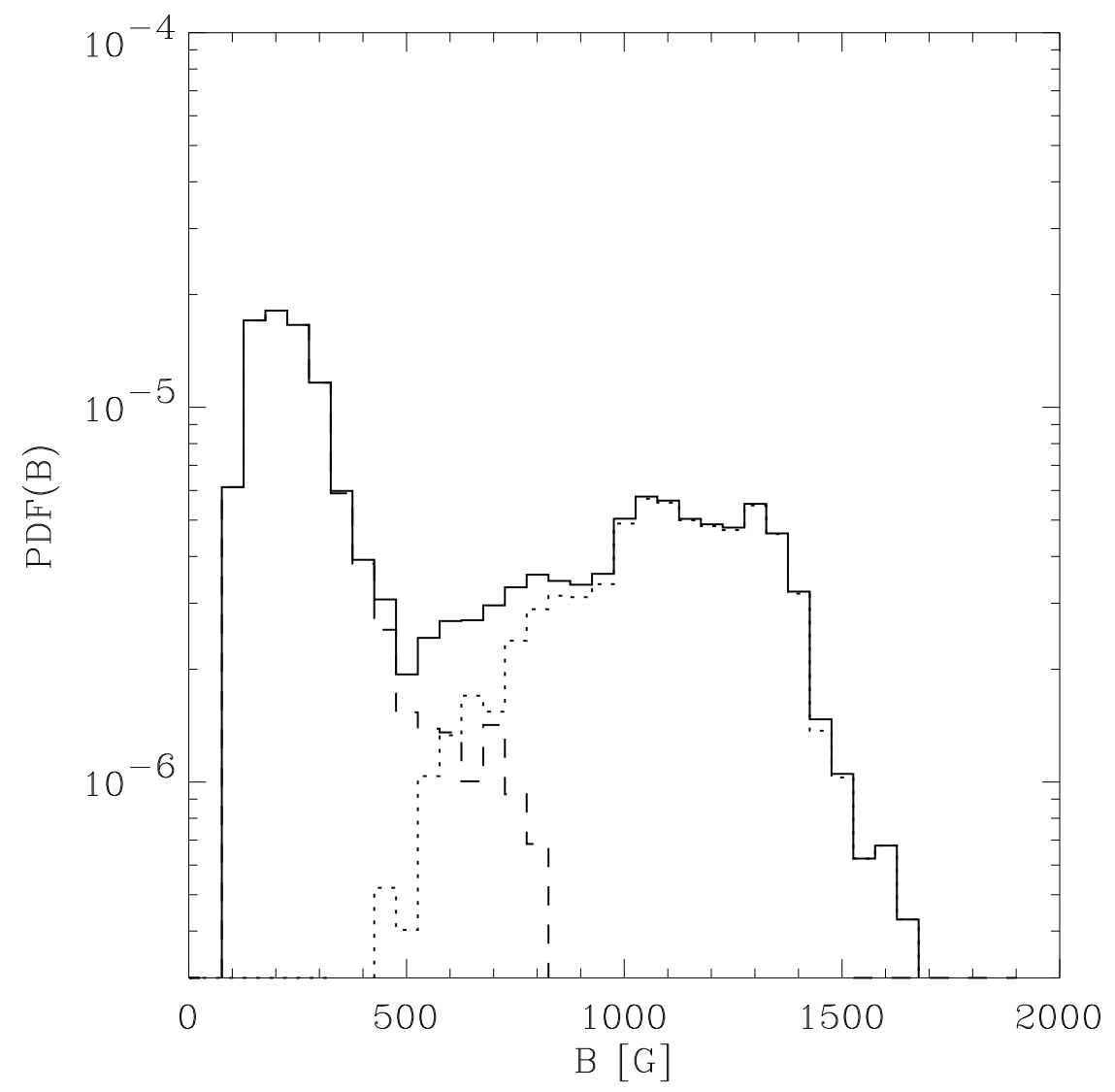

Figure 4.13: PDF of the magnetic field strength $B$ inferred from visible lines (dotted), and from IR lines (dashed). The sum of both is given by the solid line.

Table 4.7: Filling factor and magnetic flux density from PDF in Fig. 4.13. It has been computed separately for IR PDF, visible PDF, and for two different thresholds.

\begin{tabular}{lccc}
\hline \hline & $\begin{array}{c}\text { filling factor } \\
{[\%]}\end{array}$ & $\begin{array}{c}\text { flux density } \\
{[\mathrm{G}]}\end{array}$ & $\begin{array}{c}\text { fraction of flux } \\
{[\%]}\end{array}$ \\
\hline full PDF & 0.81 & 5.1 & 100 \\
IR PDF & 0.50 & 1.3 & 25 \\
Visible PDF & 0.31 & 3.8 & 75 \\
$B>500 \mathrm{G}$ & 0.4 & 4.2 & 81 \\
$B>1000 \mathrm{G}$ & 0.25 & 3 & 60 \\
\hline \hline
\end{tabular}




\subsection{MISMA inversion}

The goal of this section is the study of the simultaneous observations in the framework of a more realistic atmospheric model, able to fit the asymmetries found in all Stokes $V$ profiles, and yielding more reliable results than the ME inversion. Such a work could be performed by inverting the observed profiles under the MISMA hypothesis.

The MIcroStructured Magnetized Atmosphere (MISMA) was introduced for the first time by Sánchez Almeida et al. (1996), with the objective to reproduce all kind of asymmetric Stokes profiles present in most observations from solar structures. The basic idea of this theory is to consider an atmosphere with magnetic structures changing at scales much smaller than the mean-free-path of the photons $(\sim 100 \mathrm{~km}$ at $\tau=1)$. Figure 4.14 shows a possible scheme of a MISMA. In the quiet Sun, it can be considered as an atmosphere with many optically thin flux tubes embedded in a non-magnetic background. The magnetic structures are optically thin because their diameters are much smaller than the mean-free-path of the photons. The RTE for polarized light can be written as

$$
\frac{d \mathbf{I}}{d s}=-\mathbf{K}(\mathbf{I}-\mathbf{S})
$$

with $s$ the path length along the direction of integration. The Stokes profiles produced by a MISMA are hard to obtain from Eq. 4.7 due to the small scale of details in such an atmosphere. To solve this problem Sánchez Almeida et al. (1996) took the local average of the RTE. When the structures are much smaller than the mean-free-path, as in the case of a MISMA, it can be written as

$$
\frac{d \mathbf{I}}{d s}=\langle\mathbf{K}\rangle\left(\mathbf{I}-\mathbf{S}^{\prime}\right),
$$

with $\mathbf{S}^{\prime}=\langle\mathbf{K}\rangle^{-\mathbf{1}}\langle\mathbf{K S}\rangle$, and $\langle\ldots\rangle$ denotes an average over the micro-structures. Equations 4.7 and 4.8 are formally identical. It is well known that in order to produce asymmetric Stokes profiles integrating Eq. 4.7, one needs to introduce gradients of the line-of-sight velocity (and/or of the magnetic field). However, correlations between velocities and magnetic fields of the micro-structures destroy the symmetries of the absorption matrix $\langle\mathbf{K}\rangle$, and the solution of Eq. 4.8 results automatically in asymmetric profiles with no need of gradients (see Sánchez Almeida \& Landi Degl'Innocenty 1996).

There are some reasons to choose the MISMA inversion as a convincing method to study the IN fields. First, the MISMA scenario has become the only one which reproduces all kind of Stokes $V$ profiles observed in the quiet Sun (Sánchez Almeida \& Lites 2000). Other inversion techniques require the definition of complex models of flux tubes with a large amount of free parameters while MISMA inversions do not need additional ingredients. Finally, the upper limit for the size of the 


\section{Non-magnetic component \\ $\diamond \quad$ Magnetic component \\ $\longrightarrow$ Direction of the flow}

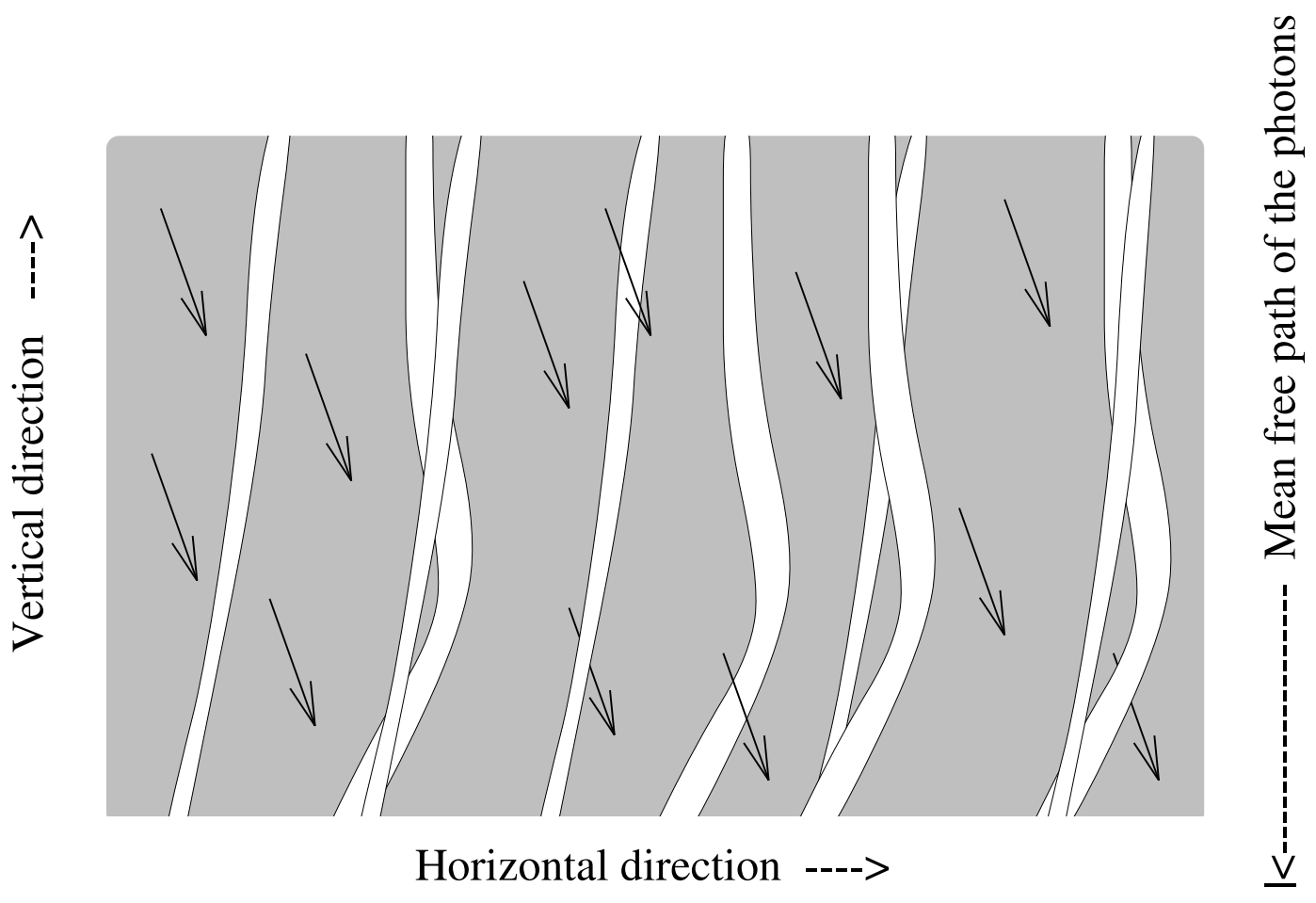

Figure 4.14: Artist's view of a MISMA, i.e., an atmosphere with properties changing in scales smaller than the mean-free-path of the photons. It shows some magnetic tubes which belong to a magnetic component embedded in a nonmagnetic downflowing background. Courtesy J. Sánchez Almeida (from Sánchez Almeida et al. 1996, Fig 7) 
small-scale magnetic structures in the quiet Sun seems to be 50-100 km (see Section 3.4.2; Khomenko et al. 2002). This number presumes one single structure in the resolution element, however it is sensible to assume that the dynamical influence of the granulation on the magnetic fields produces a distribution of very small structures. This phenomenon was already discovered in MHD simulations of the quiet Sun, like local dynamo simulations (Cattaneo 1999; Emonet \& Cattaneo 2001), or in more realistic 3D magnetoconvection (e.g., Stein \& Nordlund 2002; Vögler 2003). More arguments in favour of a microstructured atmosphere can be found in Sánchez Almeida (1998).

The inversion code under the MISMA hypothesis was developed by Sánchez Almeida (1997) and it solves Eq. 4.8 under local thermodynamic equilibrium (LTE). The inversion was carried out, at first, separately for the visible and IR pair of lines and finally, for the four lines simultaneously. The general properties of the atmosphere assumed in the inversion are those described in Sánchez Almeida \& Lites (2000):

1. There are $N$ different components, one non-magnetic and $N-1$ magnetic ( $N=3$ in the case of separated inversions and $N=4$ for simultaneous inversions). They are embedded in a background which is considered to produce unpolarized stray light.

2. The magnetic fields are vertical, along the line of sight. Since Stokes $Q$ and $U$ have very low signals it is reasonable to assume the inclination of the magnetic field to be $0^{\circ}$ or $180^{\circ}$. The inversions were performed only for Stokes $I$ and Stokes $V$.

3. The temperature of the $N$ components is the same. Since the magnetic structures are optically thin the radiative exchange between the components and background should be very efficient (Stix 2002).

4. The $N$ components are in lateral pressure balance.

5. Motions are allowed along magnetic field lines.

Table 4.8 describes the parameters used to fit the Stokes profiles. The temperatures $T_{0}\left(z_{j}\right)$ are retrieved at four fixed heights in the atmosphere and are considered the same for the $N$ components. The parameters from each component $i$ are inferred at the bottom $z_{0}$ of the integration domain. They are the magnetic field strength, the occupation fraction, the velocity and broadening parameters. The stratification of these parameters is obtained under the assumption of hydrostatic equilibrium and horizontal pressure balance. Note that the magnetic field inclination and azimuth do not appear in the table since they are fixed parameters. Finally, three parameters account for the stray light, and $\xi$ parametrizes 
Table 4.8: List of the free parameters considered in the inversion.

\begin{tabular}{cl}
\hline \hline$T_{0}\left(z_{j}\right)$ & Temperature at the four heights $z_{j\{j=0 \ldots 3\}}$ \\
$B_{m}\left(z_{0}\right)$ & Maximum magnetic field strength at $z_{0}$ \\
\hline$B_{i}\left(z_{0}\right)$ & Magnetic field strength of each magnetic component at $z_{0}$ \\
$\alpha_{i}\left(z_{0}\right)$ & Occupation fraction at $z_{0}$ \\
$U_{i}\left(z_{0}\right)$ & Bulk velocity of each component at $z_{0}$ \\
$v_{i}\left(z_{0}\right)$ & Doppler width (microturbulence) of each component at $z_{0}$ \\
$a_{i}$ & Damping coefficient of each component \\
\hline$f_{s l}$ & Fraction of stray light (i.e. from outside MISMA, but within \\
& the resolution element) \\
$U_{s l}$ & Doppler shift \\
$v_{s l}$ & Doppler width \\
$\xi$ & Macroturbulence \\
\hline \hline
\end{tabular}

the solar macroturbulence and the instrumental broadening (i.e., produced by a limited spectral resolution). The number of free parameters amounts to 20 for a three-component atmosphere. This number is just two times larger than that used in the ME inversion of the previous Section, but smaller than other inversion procedures able to fit asymmetries with flux tube models (e.g., Bellot Rubio et al. 1997; Frutiger \& Solanki 1998).

\subsubsection{Independent inversion}

The inversion was performed independently for the visible and IR pairs of lines. The MISMA models were able to fit all asymmetries with two magnetic components. All results presented now are from these inversions. A final section (Section 4.6.5) will show some successfull simultaneous inversions.

An example of fit to intensity profiles of the two pairs of lines is shown in Fig. 4.15. The observed profiles (circles) exhibit some line blends. These are a telluric line close to the red wing of Fe I $\lambda 6302.5 \AA$, a solar Fe I line at $15647.3 \AA$ and a telluric line in the red wing of Fe I $\lambda 15648 \AA$. To avoid the contamination by these blends, the weights of the Stokes $I$ profiles (and not of Stokes $V$ ) at the wavelength of the blends are set to zero ${ }^{7}$. There are also two bands of the $\mathrm{OH}$ molecule, of solar origin, close to Fe I $\lambda 15652 \AA$, however these bands are only visible in cooler solar regions than the quiet Sun (like in sunspots). The intensity profiles in Fig. 4.15 are those from class 2 . Note that Fe I $\lambda 6301.5 \AA$ and Fe I $\lambda 6302.5 \AA$ are stronger lines than Fe I $\lambda 15648 \AA$ and Fe I $\lambda 15652 \AA$. It is well known that these IR lines

\footnotetext{
${ }^{7}$ The same procedures were used in the ME inversions.
} 

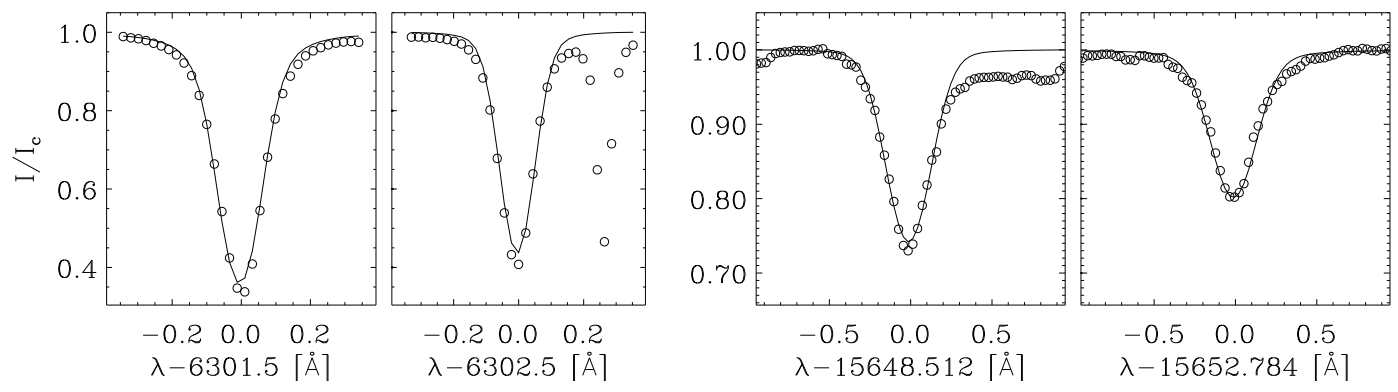

Figure 4.15: Example of fit to Stokes $I$ profiles of the four lines. The inversions were performed separately for visible and IR pairs of lines. The circles represent the observed profiles, and solid line the fit.

are formed lower in the solar atmosphere than the visible ones.

Figures 4.16 and 4.17 show the fits produced by the MISMA inversion to the same classes of Stokes $V$ profiles presented in Figs. 4.6 and 4.7. The quality of the fits is very good and all kind of profiles are reproduced. In order to make a distinction of the magnetic components, they are divided in major and minor components. The major component is that carrying the largest mass $\left(\int \alpha \rho d z\right)$, as was defined by Sánchez Almeida \& Lites (2000). The inversion gives the variation with height of the physical parameters (see Table 4.8) inferred in each class or individual pixel. From now on, all results will be referred to a single height in the atmosphere. The selected height was the base of the quiet Sun photosphere (as in Socas-Navarro \& Sánchez Almeida 2002) which can be defined as the height with

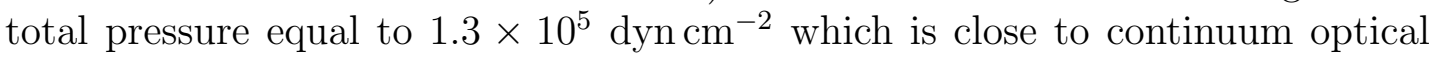
depth unity. The parameters analyzed will be the magnetic field strength and the filling factor. The study concentrates on these two parameters to enhance their importance.

The magnetic field strength inferred from the MISMA inversion in the visible and the IR is also given in Figs. 4.16 and 4.17, for each inverted class. The magnetic field of the major and the minor component are denoted as $\mathrm{B}_{1}$ and $\mathrm{B}_{2}$, respectively. It is remarkable that 4 of the 5 most abundant classes possess a strong magnetic component in the IR (i.e. with field strength larger than $1 \mathrm{kG}$ ). 


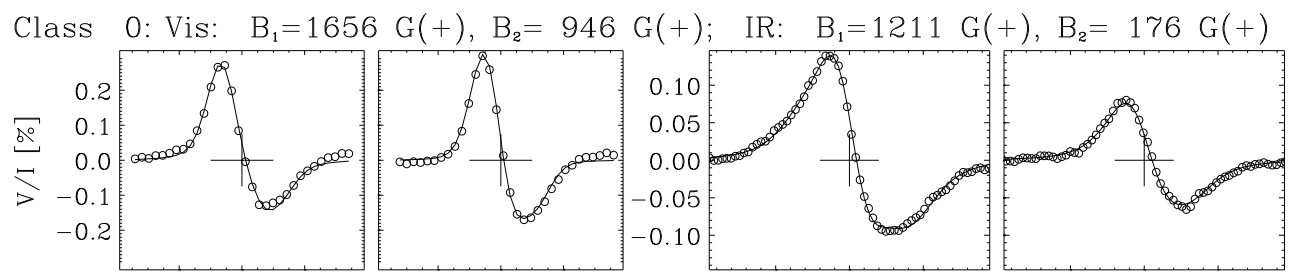

Class 1: Vis: $\mathrm{B}_{1}=1691 \mathrm{G}(+), \mathrm{B}_{2}=1296 \mathrm{G}(+) ; \quad$ IR: $\mathrm{B}_{1}=1471 \mathrm{G}(+), \mathrm{B}_{2}=395 \mathrm{G}(+)$

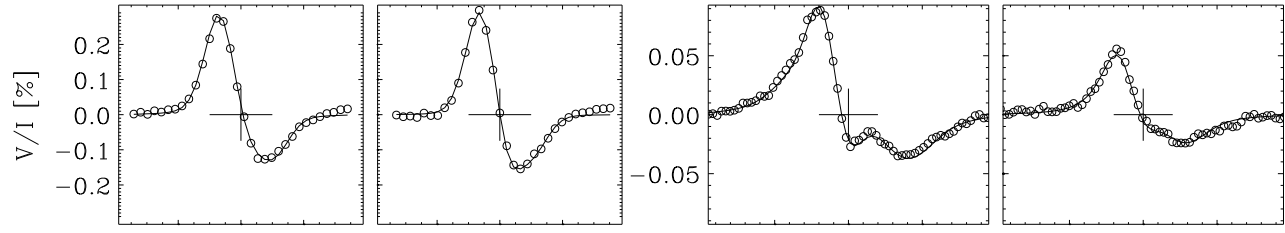

Class 2: Vis: $\mathrm{B}_{1}=1726 \mathrm{G}(+), \mathrm{B}_{2}=1259 \mathrm{G}(+) ; \quad \mathrm{IR}: \mathrm{B}_{1}=49 \mathrm{G}(+), \mathrm{B}_{2}=216 \mathrm{G}(+)$

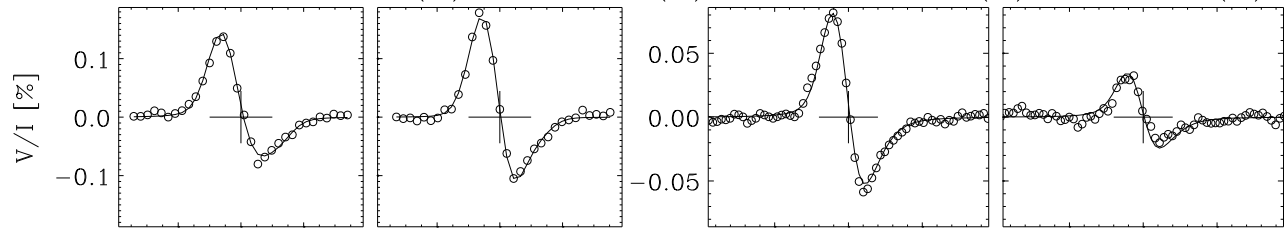

Class 3: Vis: $B_{1}=445 \mathrm{G}(+), \mathrm{B}_{2}=1732 \mathrm{G}(+) ; \quad$ IR: $\mathrm{B}_{1}=1438 \mathrm{G}(+), \mathrm{B}_{2}=261 \mathrm{G}(+)$

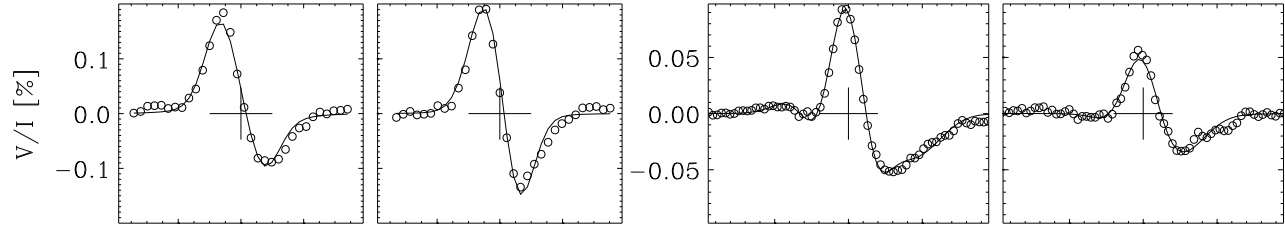

Class 4: Vis: $\mathrm{B}_{1}=1505 \mathrm{G}(+), \mathrm{B}_{2}=1488 \mathrm{G}(+) ; \quad \mathrm{IR}: \mathrm{B}_{1}=107 \mathrm{G}(+), \mathrm{B}_{2}=1165 \mathrm{G}(+)$
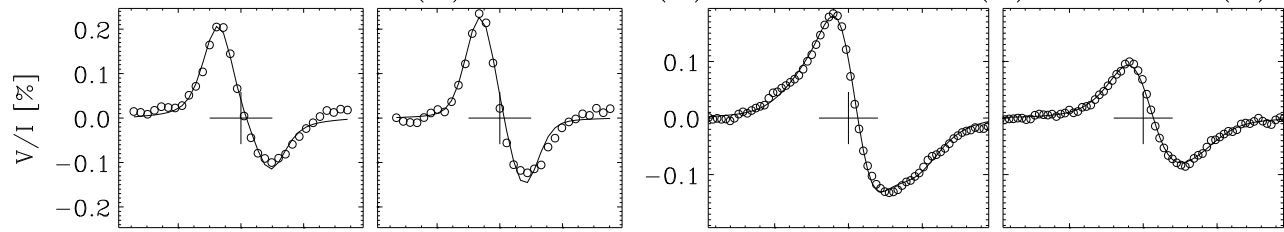

Class 5: Vis: $\mathrm{B}_{1}=1781 \mathrm{G}(+), \mathrm{B}_{2}=1375 \mathrm{G}(+) ; \quad \mathrm{IR}: \mathrm{B}_{1}=254 \mathrm{G}(-), \mathrm{B}_{2}=274 \mathrm{G}(+)$
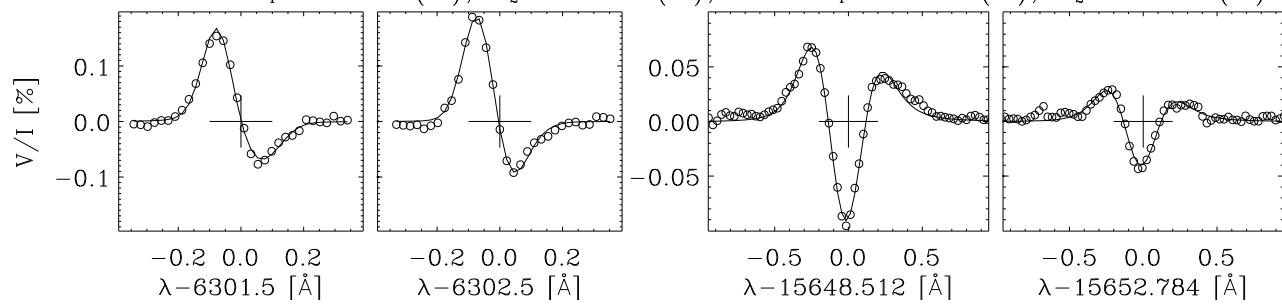

Figure 4.16: Result of the MISMA inversion of the classes presented in Fig. 4.6. Inversions were performed separately for the visible and IR pair of lines. The circles represent the observed profiles and the solid lines the fits. The polarity of the magnetic fields is indicated by ' + ' and '-' signs. 


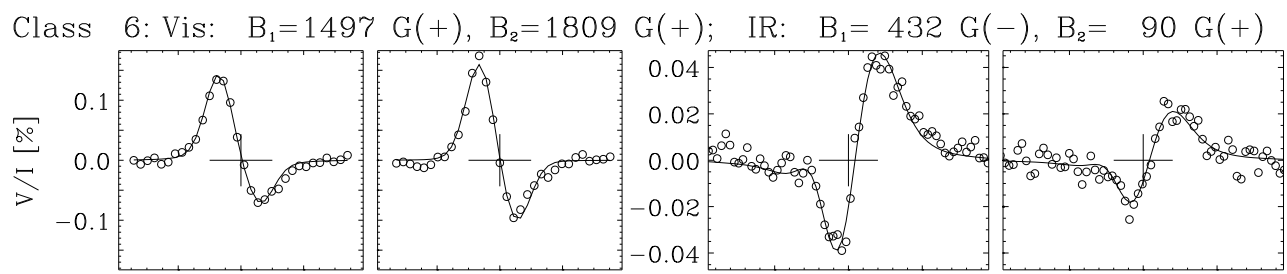

Class 10: Vis: $B_{1}=1496 \mathrm{G}(+), \mathrm{B}_{2}=1711 \mathrm{G}(+) ; \quad$ IR: $\mathrm{B}_{1}=1406 \mathrm{G}(+), \mathrm{B}_{2}=88 \mathrm{G}(+)$
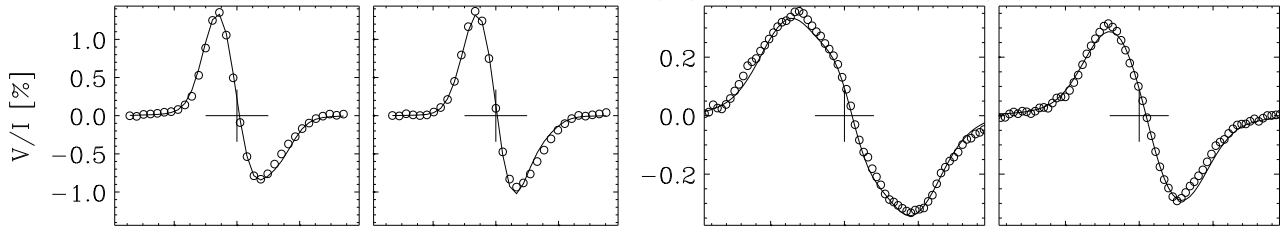

Class 13: Vis: $B_{1}=1606 \mathrm{G}(+), \mathrm{B}_{2}=415 \mathrm{G}(+) ; \quad \mathrm{IR}: \mathrm{B}_{1}=51 \mathrm{G}(+), \mathrm{B}_{2}=493 \mathrm{G}(+)$
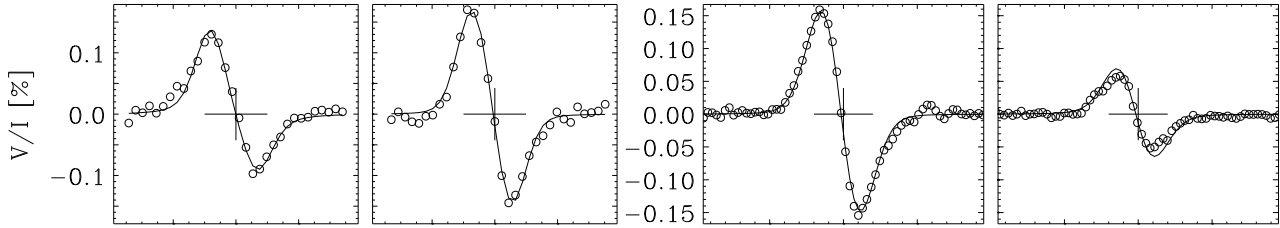

Class 20: Vis: $\mathrm{B}_{1}=1377 \mathrm{G}(+), \mathrm{B}_{2}=835 \mathrm{G}(+) ; \quad \mathrm{IR}: \mathrm{B}_{1}=201 \mathrm{G}(-), \mathrm{B}_{2}=173 \mathrm{G}(+)$
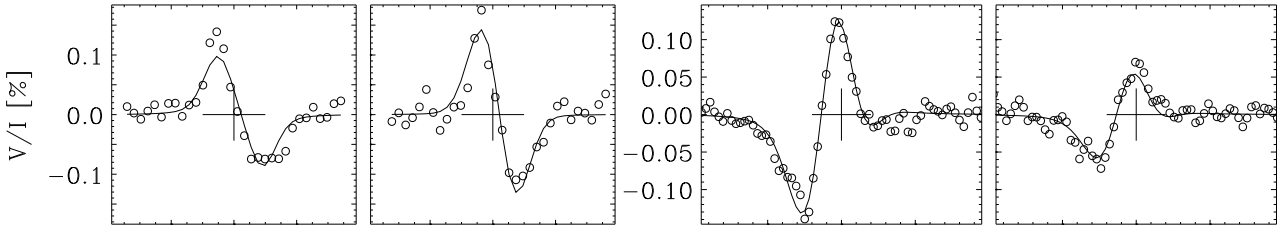

Class 30: Vis: $\mathrm{B}_{1}=276 \mathrm{G}(-), \mathrm{B}_{2}=426 \mathrm{G}(+) ; \quad \mathrm{IR}: \mathrm{B}_{1}=1135 \mathrm{G}(-), \mathrm{B}_{2}=199 \mathrm{G}(+)$
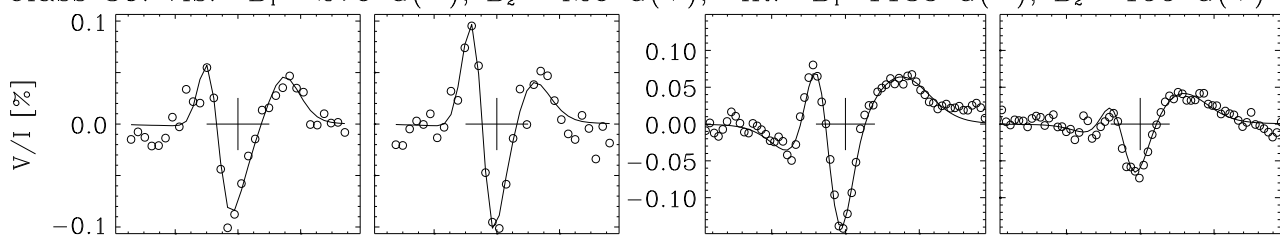

Class 36: Vis: $\mathrm{B}_{1}=457 \mathrm{G}(-), \mathrm{B}_{2}=406 \mathrm{G}(+) ; \quad \mathrm{IR}: \mathrm{B}_{1}=556 \mathrm{G}(-), \mathrm{B}_{2}=364 \mathrm{G}(+)$
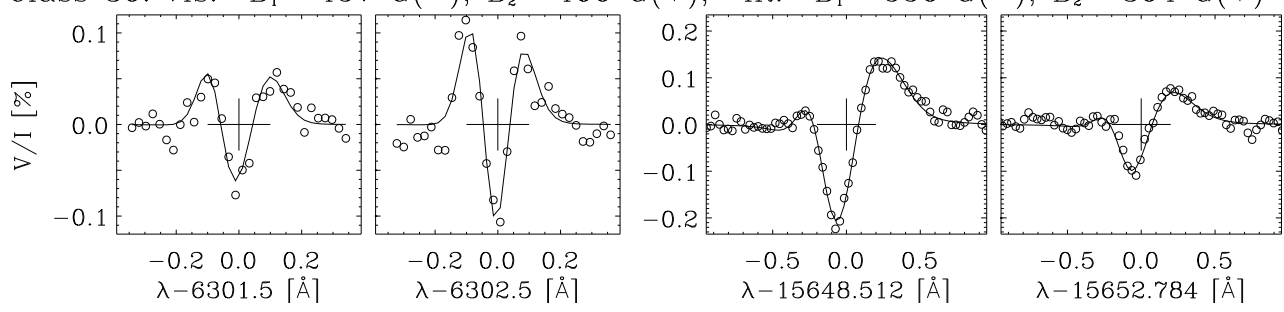

Figure 4.17: Result of the MISMA inversion of the classes presented in Fig. 4.7. Inversions were performed separately for the visible and IR pair of lines. The circles represent the observed profiles and the solid lines the fits. The polarity of the magnetic fields is indicated by ' + ' and '-' signs. 

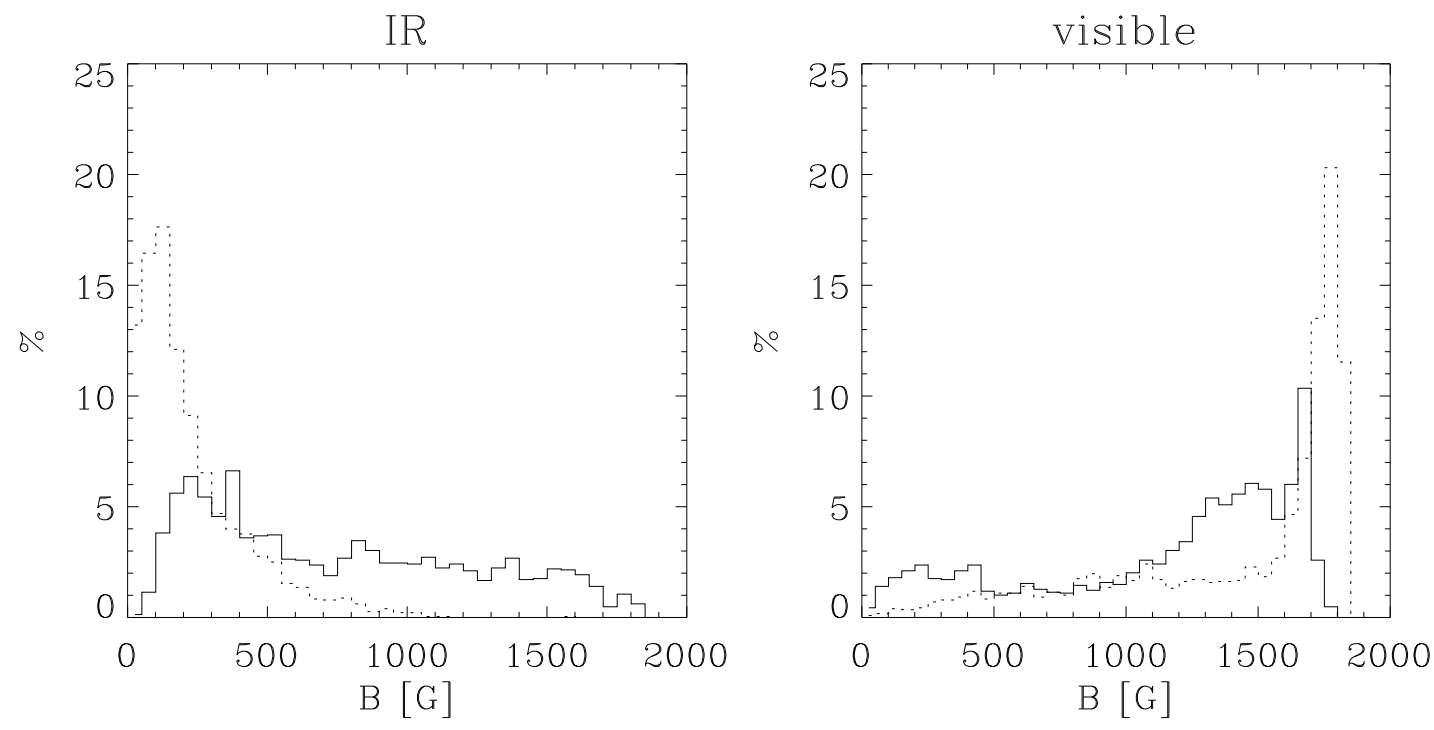

Figure 4.18: Comparison between results from MISMA inversion for the visible and IR lines. Histograms of $B$ (magnetic field strength) for IR (left) and for the visible (right). Solid and dotted lines represent the major and minor magnetic components, respectively.

\subsubsection{Magnetic field strength}

Figure 4.18 displays the histograms with the distribution of the magnetic field strength from the inversion of IR (left) and visible lines (right). They show the percentage of pixels within bins of $50 \mathrm{G}$ of intrinsic field of the major and minor component (solid and dotted lines, respectively).

In the visible histogram, the major component tends to have weaker fields than the minor. The average magnetic fields are $1150 \mathrm{G}$ and $1415 \mathrm{G}$ for the major and minor component, respectively. This histogram is similar to that obtained by Sánchez Almeida \& Lites (2000; Fig 14), however the one obtained here possesses an extended tail of fields weaker than $500 \mathrm{G}$. Table 4.9 presents the percentage of pixels under analysis with magnetic fields above various thresholds obtained with MISMA and ME inversions. $18 \%$ of the pixels show fields weaker than $500 \mathrm{G}$ in the major or minor component. All pixels from which weak fields were measured showed very low polarization signals, hence there are two possibilities: 1) it is a real phenomenon associated with the lowest polarization signals, or 2) it is an effect of the noise, and the MISMA inversion guesses weak fields where it should find strong fields.

In contrast to the visible, the IR histogram (left panel in Fig. 4.18) presents a major magnetic component with stronger fields than the minor component. In 
Table 4.9: Percentage of pixels under analysis with magnetic field strength above three different thresholds inferred from the MISMA inversion (for major or minor component), as well as from the ME inversion, for IR and visible lines.

\begin{tabular}{lcccc}
\hline \hline & $\begin{array}{c}\text { IR (MISMA) } \\
{[\%]}\end{array}$ & visible (MISMA) & IR (ME) & visible (ME) \\
& {$[\%]$} & {$[\%]$} & {$[\%]$} \\
\hline$B>500 \mathrm{G}$ & 65 & 82 & 17 & 98 \\
$B>750 \mathrm{G}$ & 50 & 80 & 9 & 90 \\
$B>1000 \mathrm{G}$ & 32 & 77 & 2 & 60 \\
\hline \hline
\end{tabular}

this case, the average magnetic fields were $750 \mathrm{G}$ and $225 \mathrm{G}$ for major and minor magnetic component, respectively. There is a large number of pixels with strong fields in comparison with the results of the ME inversion (see Table 4.9). 50\% of the analyzed pixels contain magnetic fields larger than $750 \mathrm{G}$ when the MISMA inversion is applied to the IR profiles. They were obtained from the profiles with larger polarization signals.

The main reasons to find strong fields with the IR lines were already presented in Section 2.3. The polarization signals in the Stokes $V$ profiles of the IR lines are spread on a wide range of wavelengths when the magnetic field is much stronger than the magnetic saturation of the line ( $400 \mathrm{G}$ for Fe I $\lambda 15648 \AA)$. The cause of this can be horizontal gradients of magnetic fields produced by a continuous distribution of intrinsic fields (Figs. 2.5 and 2.6). The other possibility is the presence of a vertical gradient of field strength (Sánchez Almeida \& Lites 2000). The last one is the only effect playing a role in the current results, since the MISMA model used in the inversion takes into account only two magnetic components and not a continuous distribution.

The following test was carried out to show this phenomenon. A synthesis of Stokes $V$ for Fe I $\lambda 15648 \AA$ was done for two identical MISMA models but with the only difference in the magnetic field strengths. Figure 4.19 shows the variation of the intrinsic field (left panel) for the two models, with strong field (M1) and a four times smaller field (M2). The magnetic fields at the base of the photosphere were $1400 \mathrm{G}$ and $350 \mathrm{G}$, respectively. The right panel shows the filling factor of the magnetic components which was the same for both models, and the occupation of the non-magnetic component. Figure 4.20 displays the result of the synthesis of the two models (left panel). Even, when the magnetic flux is four times larger for $\mathrm{M} 1$ than for $\mathrm{M} 2^{8}$, the resulting profile of the first (dashed line) has a maximum of the lobes two times smaller than the profile synthesized with the last (solid line).

\footnotetext{
${ }^{8}$ Both have the same filling factor but M1 has a four times stronger intrinsic field than M2 and the flux $\propto \alpha B$, hence the flux is four times larger for M1.
} 

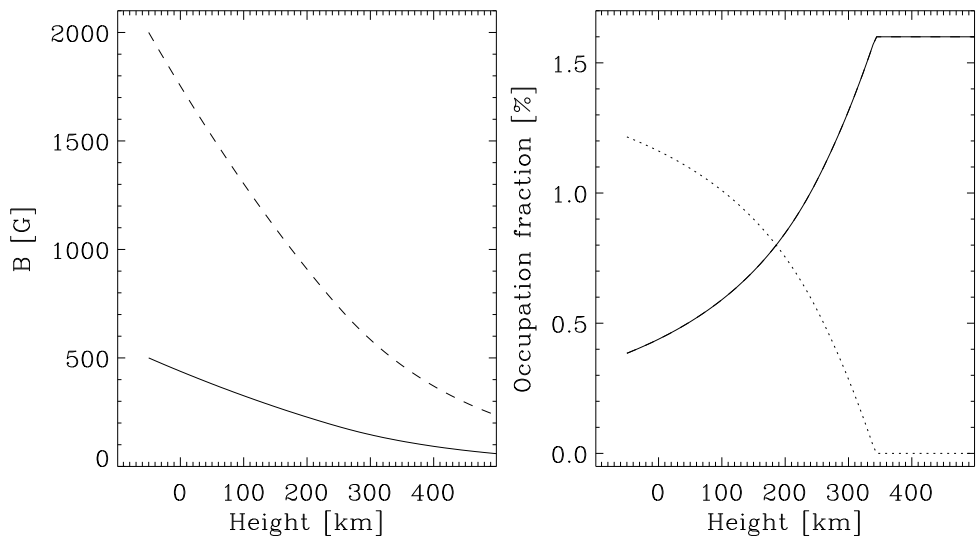

Figure 4.19: Height variation of the magnetic field strength (left) and filling factor (right) for the model MISMA yielding Stokes $V$ profiles in Fig 4.20. Both strong and weak magnetic components (dashed and solid line) have the same filling factor and the dotted line correspond to the occupation of the non-magnetic component
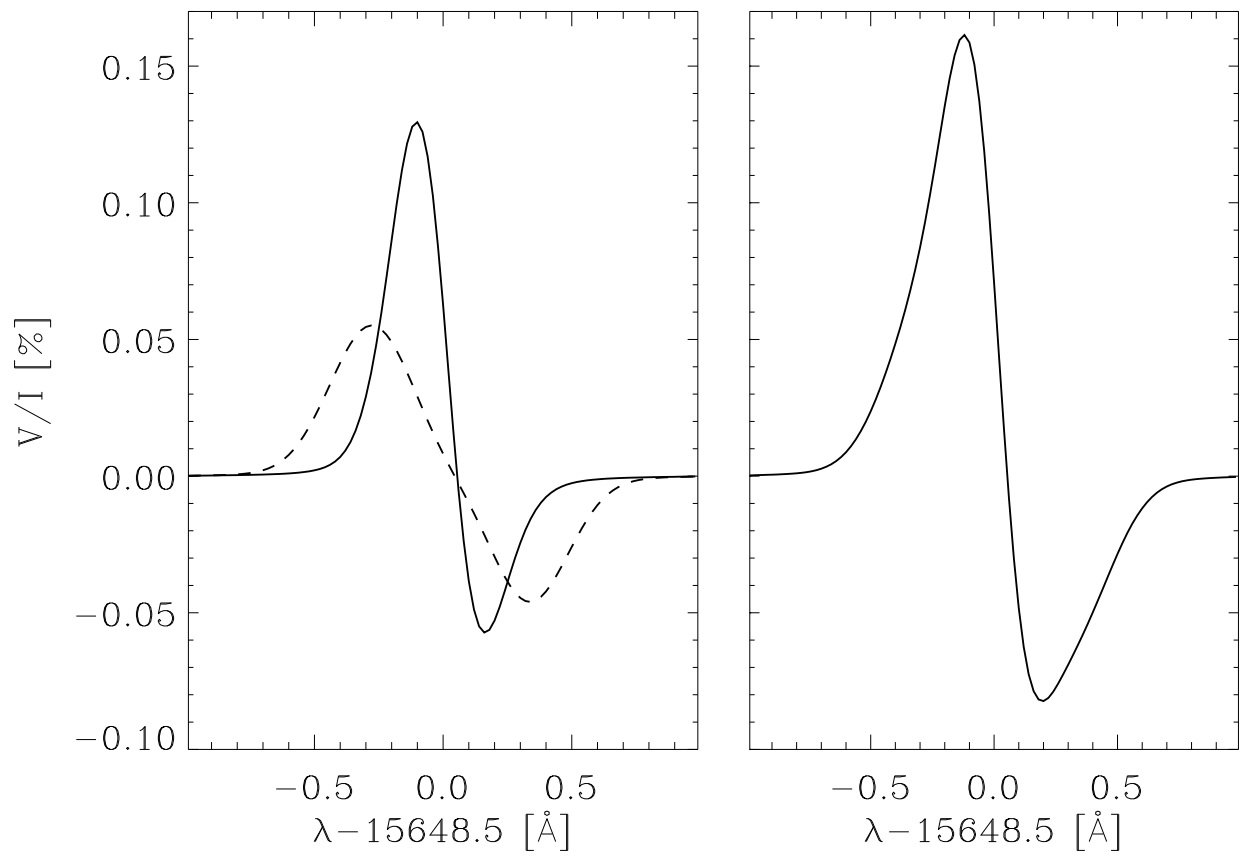

Figure 4.20: Synthetic Stokes $V$ profiles of Fe I $\lambda 15648 \AA$ from a MISMA model. Left: profiles for the weak (solid) and the strong (dashed) magnetic field stratification in Fig. 4.19. Right: sum of both weak and strong component. Note that both components have the same filling factor. 
The area of the absolute value of Stokes $V\left(A=\int_{-\infty}^{+\infty}\left|V_{\lambda}\right| d \lambda\right)$ is almost the same in both profiles. This area can be considered as the polarization contribution of the magnetic structure. Since Fe I $\lambda 15648 \AA$ is magnetically saturated for an intrinsic field stronger than 300-400 G (see Section 2.6), it is consistent that the polarization signal (the area of $|V|$ ) is the same for both profiles. The polarization signals of the profile from M1 are spread out over a larger range of wavelengths than the $V$ profile from M2 due to the strong gradient of the magnetic field in the vertical direction (see left panel of Fig. 4.19).

The combination of both profiles is presented in the right panel of Fig. 4.20. It is very similar to some of the most abundant profiles (see classes 0 and 4 in Fig. 4.16). The profile with strong field is hidden by the profile with weak field. When the splitting is used to compute the field strength one finds fields of $900 \mathrm{G}$ and $330 \mathrm{G}$ for profiles from M1 and M2 respectively. Due to the dominance of the weak field profile the splitting of the sum of both contributions yields a magnetic field of $450 \mathrm{G}$. The only way to infer any strong field is by fitting the asymmetries and polarization tails off line center of the Stokes $V$ profile with a model with two magnetic field components. With the reminder that in this example both components have the same occupation, one may now see that it is feasible to find profiles resulting from strong fields in the major component (e.g., class 0).

One may be tempted to believe that the spread in wavelength of the polarization signals and the reduction of the extrema of the $V$ profiles of Fe I $\lambda 15648 \AA$ are exaggerating and model dependent. However, there is an observational argument in favour of the above interpretation. When the profiles corresponding to network (category w4 defined in Section 4.5.1) were inverted with the ME code the resulting flux obtained from visible lines was four times larger than when it was inferred from IR lines (see Table 4.5). The IR Stokes $V$ profiles from network (class 10) are very broad due to the spread of polarization, and a ME inversion computes a flux much lower than the real one. Since visible lines are not affected by this phenomenon their ME inversion gives a more reliable flux. This can be considered as the first observational proof that the $V$ profiles of Fe I $\lambda 15648 \AA$ have their polarization signals spread over the wavelengths, thus can be hidden very easily by a weak component. When the network profiles are inverted with the MISMA code the resulting flux was very similar for both, IR and visible lines. This result gives more consistency to the MISMAs, however such profiles could also be successfully inverted by a model of magnetic flux tube (e.g., Bellot Rubio et al. 1997; Frutiger \& Solanki 1998).

A test of consistency of the results is to check whether the strong magnetic field strengths obtained from inversion of IR lines are related with those found from the visible lines. The left panel of Fig. 4.21 displays the magnetic field of the major component in the IR as a function of the average field strength of the two components in the visible. The average fields were weighted with the filling 

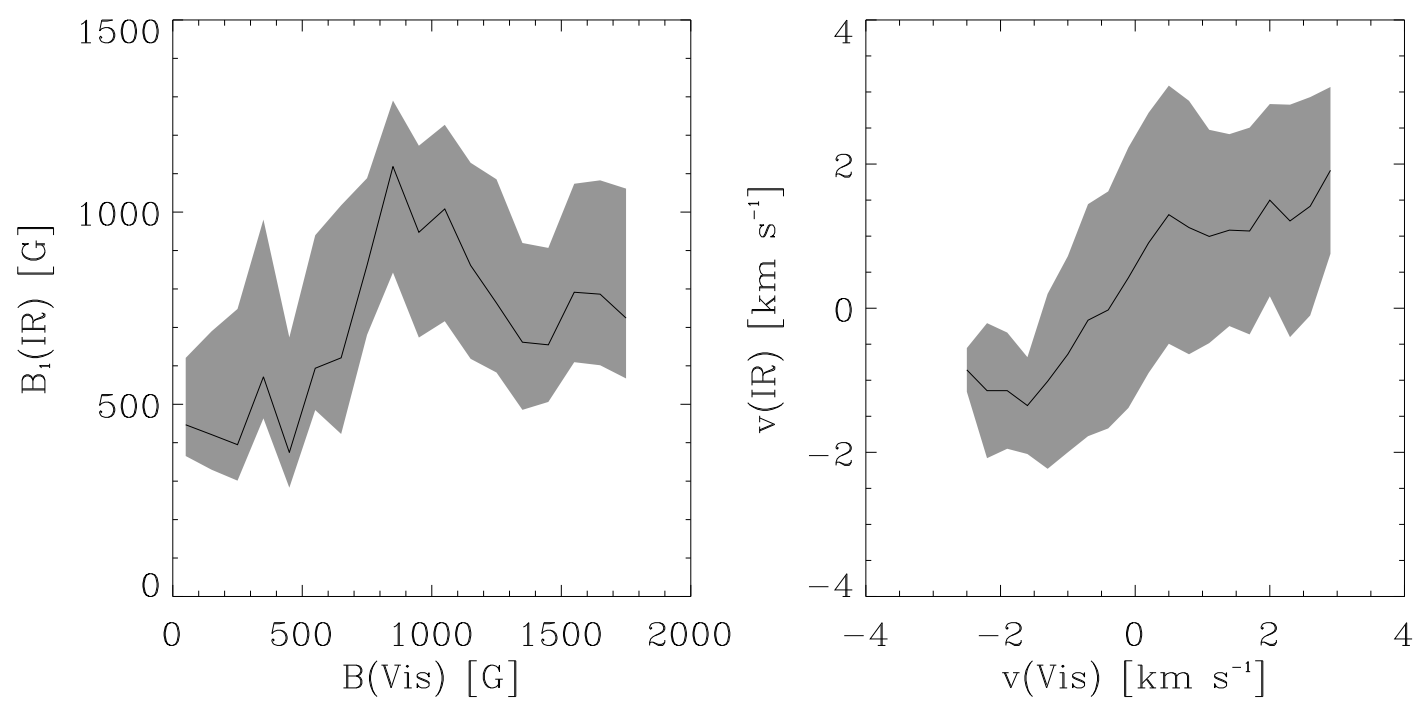

Figure 4.21: Magnetic field strength from the major component in the IR as a function of the average field strength in the visible (left) and velocity from the major component in the IR vs. velocity in the major component of the visible (right). The shaded areas represent the standard deviation.

factor of each component. There is a dependence of the IR field to increase with the visible intrinsic field strength. The correlation can be associated with the fact that both visible and IR lines are tracing the same magnetic structure with strong fields and the MISMA code is able to infer the field strength from the same structure for both pairs of lines. The dependence is interrupted at intermediate fields, i.e. between $500 \mathrm{G}$ and $1200 \mathrm{G}$, due to the few pixels with this magnetic fields in the visible.

The right panel of Fig. 4.21 shows the relation of the velocities of the major component of the IR and of the major component of the visible. Contrary to the similar plot for the ME inversion (right plot in Fig 4.12) the velocities are now obviously correlated. Since the velocities are too large to be associated with the 5min oscillations, this is another evidence that the major components with strong magnetic fields from the IR lines correspond to the same magnetic structures traced by the visible lines.

Figure 4.22 shows the maps of magnetic field strength for the major component from the IR (left) and of the average field strength from the visible (right). On the one hand, the map obtained from visible lines is very similar to that obtained from the ME inversion (Fig 4.8) but with stronger fields. On the other hand, the map of intrinsic field obtained in the IR shows many patches with fields stronger than $1 \mathrm{kG}$. These patches exhibit the strongest polarization signals. 

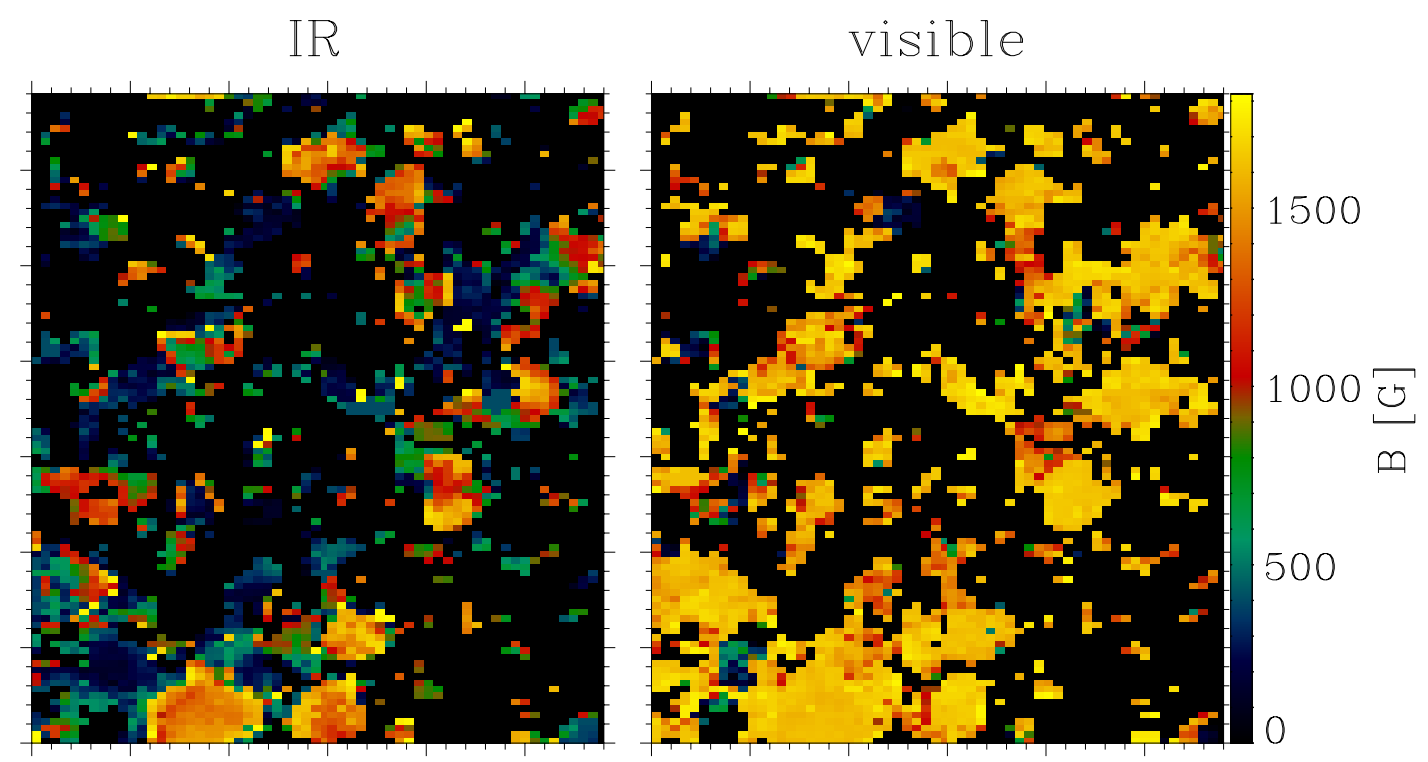

Figure 4.22: Maps of the modulus of the magnetic field strength at the base of the photosphere, obtained from MISMA inversion for the major component in the IR (left) and the weighted average between the components in the visible lines (right). Separation between tickmarks in arcsec. Both maps are equally scaled, and black represents pixels with signals too low to be analyzed.

In IR profiles with weak signals the MISMA code is unable to find the strong component because the noise is hiding the long tails expected from Fig. 4.20 and the only detectable signals stem from areas which represent only the weak field part of the distribution.

\subsubsection{Magnetic flux and filling factor}

The top panels of Fig. 4.23 display the maps of the total filling factor of the magnetic components derived from IR (left) and visible lines (right). Across the FOV, the IR lines have larger filling factors. The values are in the range of $0.1 \%$ up to $5-6 \%$. Bellow $0.1 \%$ the polarimetric signals were too noisy to attempt an inversion. The maps of the magnetic flux are presented in the bottom panels of Fig. 4.23. The map retrieved from inversion of the IR lines is very similar to that obtained for the visible lines. When the flux was inferred with a ME inversion (bottom panels in Fig. 4.10) a huge difference resulted between the magnetic flux maps from the IR and the visible lines. This difference was the consequence of 

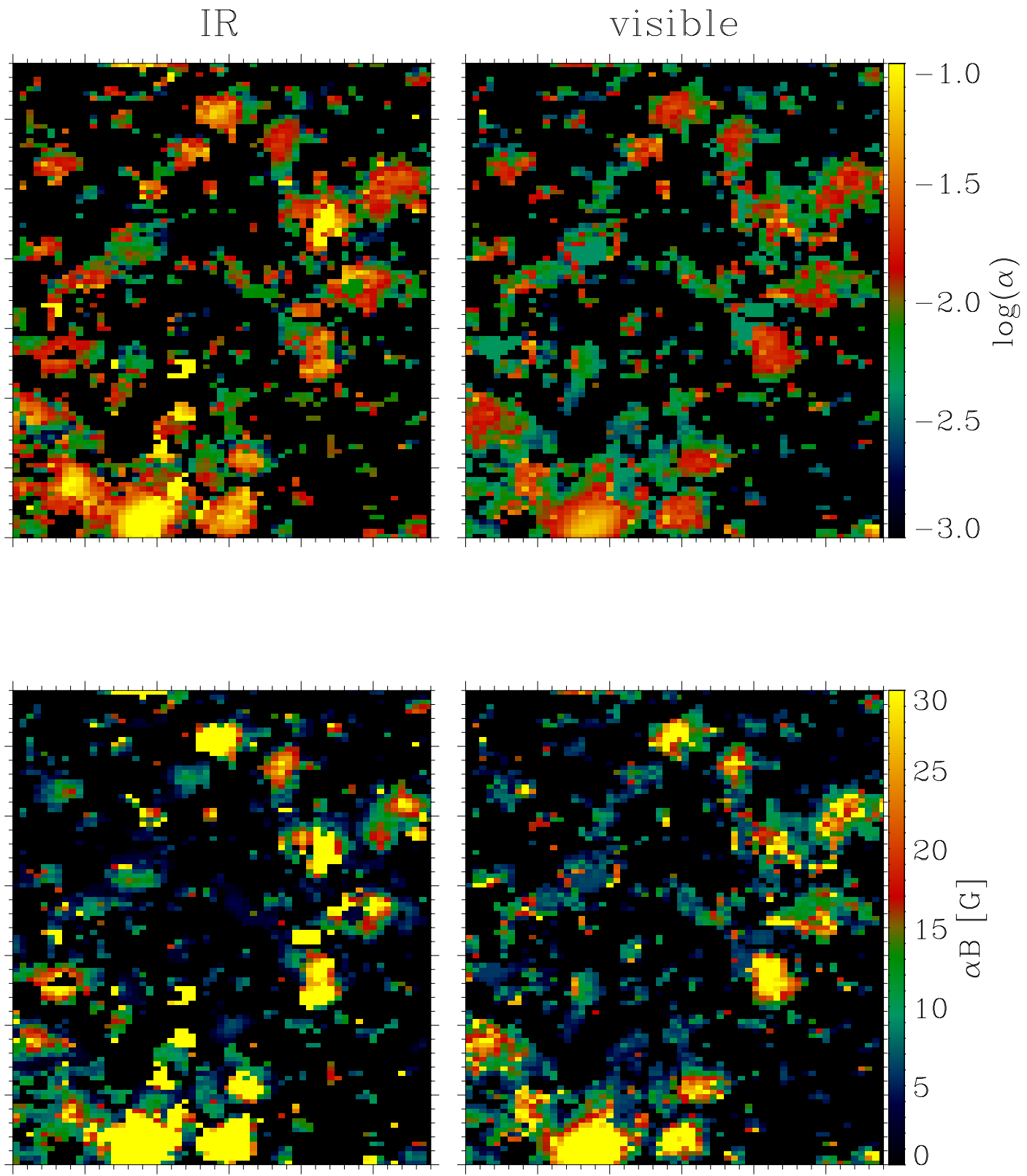

Figure 4.23: Maps of the filling factor in logarithmic scale (log $\alpha$, top panels) and of the magnetic flux density ( $\alpha B$, bottom) obtained from MISMA inversion in IR (left) and visible (right). Separation between tickmarks in arcsec. Both pairs of maps are equally scaled, and black is at pixels with too low polarimetric signals. 


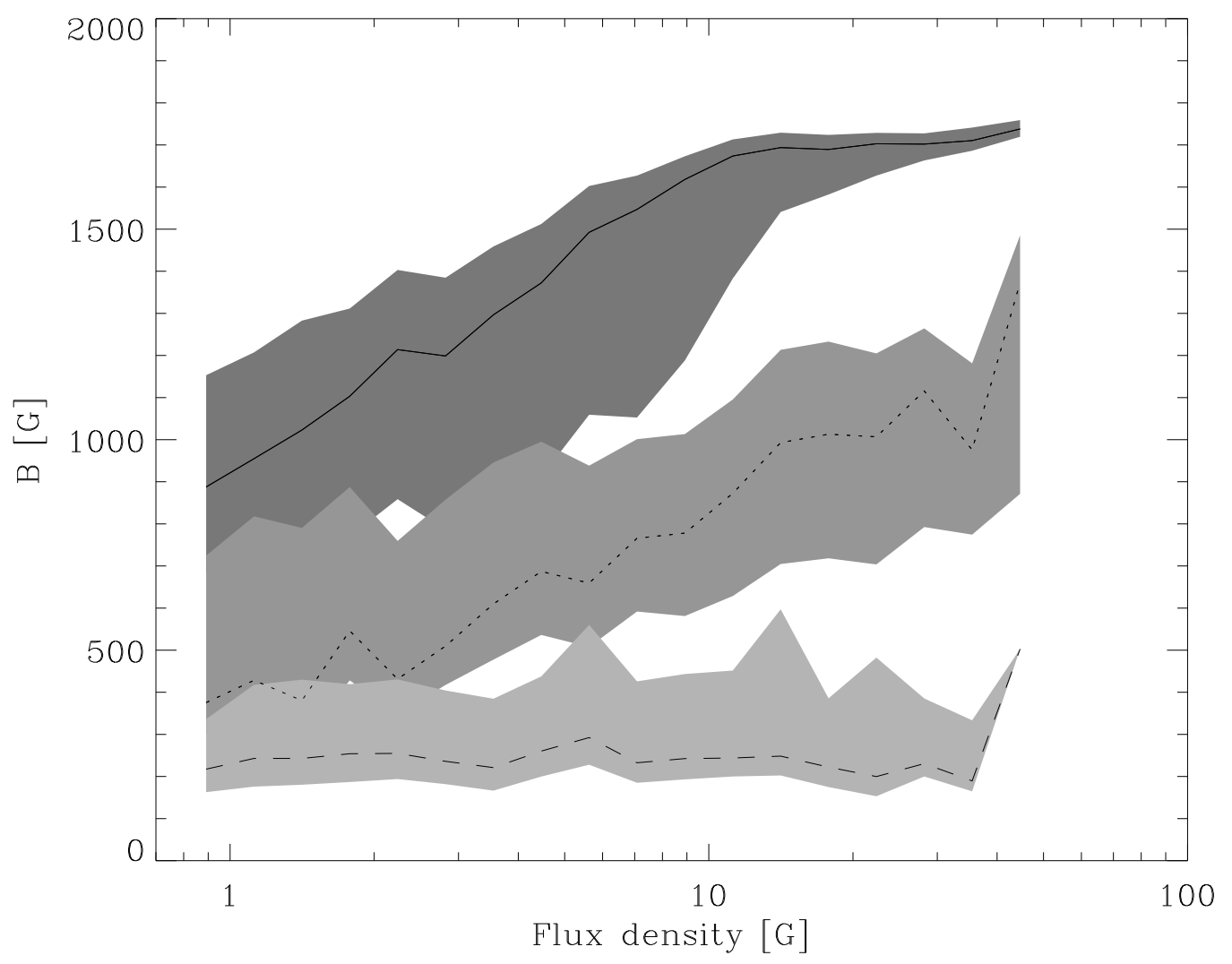

Figure 4.24: Magnetic field strength as a function of the magnetic flux density. The averages of the two components obtained in the visible are given by the solid line, and of the major and minor IR components by the dotted and dashed lines. The shaded areas give the plus-minus standard deviation.

the use of a single component model which only retrieves the magnetic field of the weak component in the IR since the strong field contribution to the Stokes $V$ profiles were hidden by the weak field contribution. Two factors play a role in the consistency and the equivalence of the maps of magnetic flux obtained from visible and IR lines. First, the MISMA inversion is able to find both the strong and the weak component contributing to the IR profiles in a high number of the pixels (see Table 4.9). These are the pixels with the strong polarimetric signals. Second, the flux obtained from visible is mainly from the strong component which carries most of the magnetic flux.

Figure 4.24 shows the variation of the magnetic field strength with the magnetic flux density. The average of the visible magnetic components (solid line), and for the major and minor IR components (dotted and dashed lines) are given. 
The shaded areas represent the variations. There is a strong tendency of the intrinsic fields obtained from the visible lines to weaken when the magnetic flux decreases, as it was already observed in the ME inversions (Fig. 4.11 a). Sánchez Almeida \& Lites (2000) obtained a smaller dependence, however their lower limit of flux density was $10 \mathrm{G}$ while in this work the lowest detectable flux could be decreased down to $1 \mathrm{G}$ due to the better polarimetric sensitivity. On the one hand, the explanation of this effect (see Section 4.6.2 and Sánchez Almeida 2000) is the dependence of the trasparency of the magnetic components on the magnetic flux. More transparent structures produce Stokes $V$ profiles originated from lower layers of the solar atmosphere and therefore from stronger fields. On the other hand, this strong dependence can also be explained by a magneto-dynamic effect. Smaller structures and consequently those structures which harbour lower flux cannot concentrate enough magnetic fields to enhance the intrinsic fields to higher values.

The major component of the models obtained from the IR lines exhibits, similarly as from the visible lines, a strong dependence of the intrinsic field strength on the average flux density, with the difference that the mean fields are smaller than in the visible. The dependence can be ascribed to the two reasons described above, but still a third effect should be mentioned. The MISMA code has problems to detect the strong field component from profiles with low polarimetric signals, since noise hides the contributions of such magnetic fields in favour to the weak fields. If this is the main reason for the dependence and since the intrinsic field grows with the flux, even for the higher flux densities the inversion is biased towards the weak fields. The conspicuous fact that the magnetic field of the minor component inferred from IR lines shows no dependence at all on the magnetic flux indicates that the increase of the transparency with the flux may play no role for weak fields and that the other two effects are the only taking place.

\subsubsection{Probability Density Function (PDF)}

The distribution of the magnetic field strength inferred from the MISMA inversion was computed in the same way as for the ME inversion (see Section 4.5.3). Figure 4.25 displays the PDF of the magnetic fields derived from IR observations (solid line), from visible observations (dotted) and the total PDF inferred from ME inversions from Fig. 4.13 (dashed).

The PDF derived from visible observations is mainly concentrated at strong intrinsic fields and shows a further, smaller concentration at weaker fields. The intermediate gap of intrinsic fields with low PDF between 500 and $1200 \mathrm{G}$ was expected from the histogram of Fig. 4.18. It was already pointed out in Section 4.6.2 that this phenomenon can be an artifact of the MISMA inversion since the weak fields were retrieved from profiles with the lowest polarization signals. 


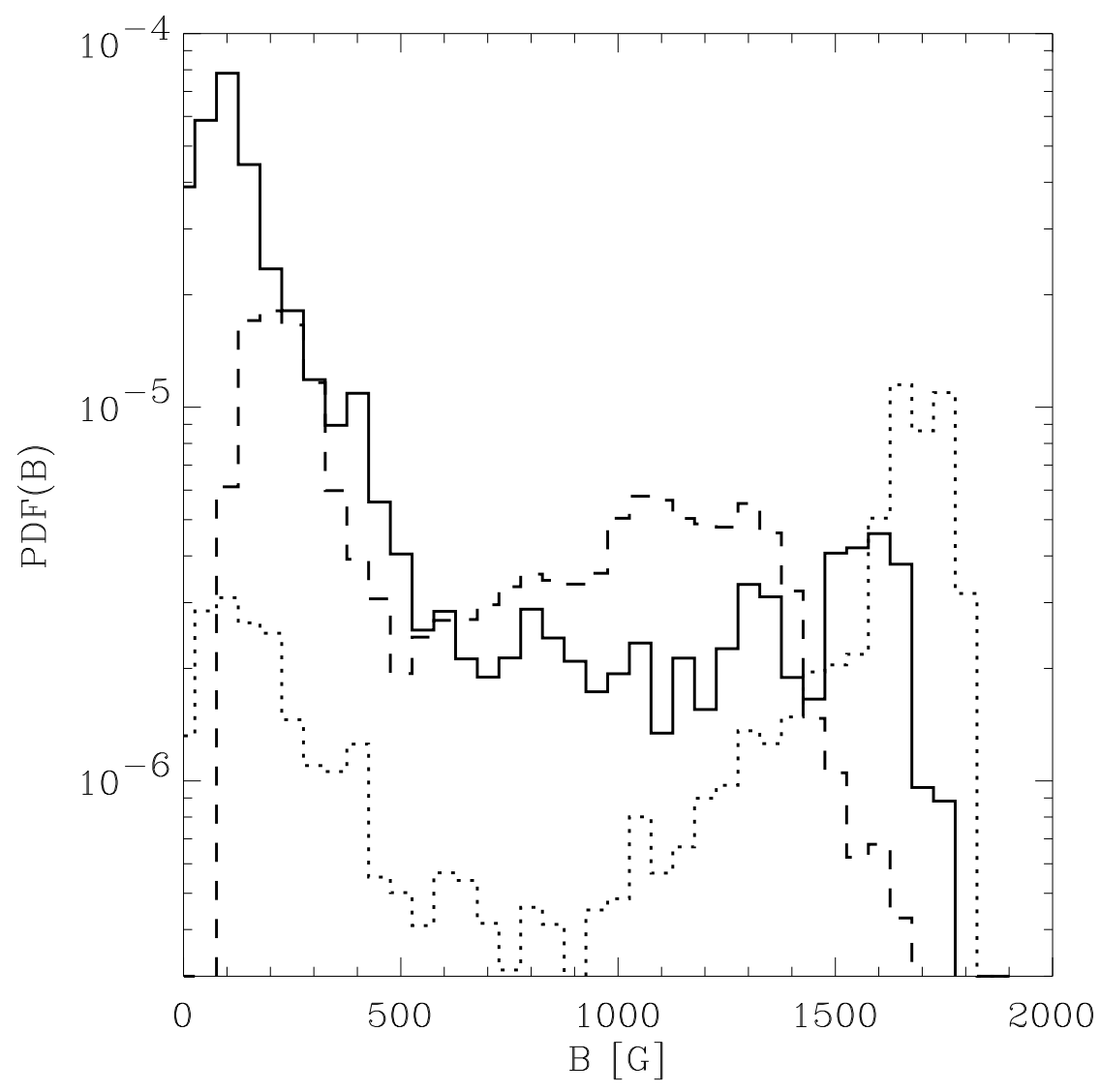

Figure 4.25: PDF of the magnetic field strength $B$ obtained from the inversion under the MISMA hypothesis for IR lines (solid) and visible lines (dotted). The dashed line is the total PDF from ME inversion (Fig. 4.13).

However, there exists the posibility that the MISMA inversion overestimates some of the stronger intrinsic fields which produces the gap. The solution of this problem must be deferred to further studies.

The PDF inferred from IR observations is quite similar to the sum of the PDFs obtained from ME inversions of IR and visible lines. Table 4.10 is a guide to compare and analyze the PDF. It shows the filling factor, the flux density, the fraction of flux and the fraction of magnetic energy for the three PDFs in Fig. 4.25 in three different ranges of magnetic field strength, i.e., for $B>B_{0}=0 \mathrm{G}$ (total PDF), $B>B_{0}=500 \mathrm{G}$ and $B>B_{0}=1000 \mathrm{G}$. The flux density and the density 
Table 4.10: Filling factor, magnetic flux density, fraction of the total flux and fraction of the total magnetic energy from the PDFs in Fig. 4.25. They were computed separately for the IR PDF, visible PDF, PDF from ME inversion (Fig. 4.13) with two different thresholds. Note that the values are relative to the total the FOV.

\begin{tabular}{lcccc}
\hline \hline & $\begin{array}{c}\text { filling factor } \\
{[\%]}\end{array}$ & $\begin{array}{c}\text { flux density } \\
{[\mathrm{G}]}\end{array}$ & $\begin{array}{c}\text { fraction of flux } \\
{[\%]}\end{array}$ & $\begin{array}{c}\text { fraction of energy } \\
{[\%]}\end{array}$ \\
\hline PDF (IR) & & & 100 & 100 \\
total & 1.80 & 5.7 & 64 & 91 \\
$B>500 \mathrm{G}$ & 0.30 & 3.7 & 50 & 79 \\
$B>1000 \mathrm{G}$ & 0.20 & 2.8 & & \\
\hline PDF (Vis) & & & 100 & 100 \\
total & 0.38 & 4.6 & 97 & 99.5 \\
$B>500 \mathrm{G}$ & 0.29 & 4.5 & 94 & 98 \\
$B>1000 \mathrm{G}$ & 0.26 & 4.3 & & 100 \\
\hline PDF (ME) & & & 100 & 95 \\
total & 0.81 & 5.1 & 82 & 78 \\
$B>500 \mathrm{G}$ & 0.40 & 4.2 & 60 & \\
$B>1000 \mathrm{G}$ & 0.25 & 3.0 & \multicolumn{4}{c}{} \\
\hline \hline
\end{tabular}

of the magnetic energy can be identified with

$$
\begin{gathered}
\langle B\rangle=\int_{B_{0}}^{\infty} B \cdot P D F(B) d B, \\
\frac{\left\langle B^{2}\right\rangle}{8 \pi}=\frac{1}{8 \pi} \int_{B_{0}}^{\infty} B^{2} \cdot P D F(B) d B .
\end{gathered}
$$

The first important result is that the flux density obtained from IR is $5.7 \mathrm{G}$. In contrast to the results from ME inversions (Table 4.7) this flux density of the IR PDF is larger than the flux from the visible PDF. Such a result is consistent because the MISMA inversion was able to retrieve strong and weak fields from IR profiles while the only regime of fields derived from the visible lines was that of strong fields. The next remarkable point is the much higher filling factor of the IR magnetic components compared to the ME inversion. This was $1.8 \%$ of the FOV in comparison with the $0.8 \%$ of the latter. However, the occupation of the strong fields $(B>500 \mathrm{G})$ of the PDF inferred from IR lines is consistent with the respective values of the visible PDF and the PDF from ME inversion. Thus, the excess of occupation takes place in the weak field regime. The flux density inferred from MISMA inversions of the IR lines for weak fields $(B<500 \mathrm{G})$ was 
two times larger than those from ME inversion, and the filling factor was three times larger. The reason of this differences is, on the one hand, the presence of many mixed polarities in the MISMA inversion of IR profiles. $40 \%$ of the analyzed pixels contain magnetic components with opposite polarities which were underestimated by the ME inversion. On the other hand, the MISMA inversion technique was able to retrieve weaker fields than the ME inversion (note that the PDF is large for fields close to zero). The flux density computed for strong fields $(B>500 \mathrm{G})$ was $3.7 \mathrm{G}$ in the PDF derived from IR lines, and $4.5 \mathrm{G}$ in the PDF from visible lines. Thus $80 \%$ of the flux expected to be in the strong fields by visible line analysis were detected from the IR lines. The remaining $20 \%$ of flux may be hidden in the IR profiles with low polarization signals which could not be inferred by the MISMA code (see earlier Sections).

In summary, the above results establish that the use of only the IR lines to retrieve the distribution of intrinsic fields yields a PDF close to reality but still biased to weak fields. One needs both pairs of lines in order to obtain a reliable PDF. Nevertheless, it is remarkable that the PDF derived from IR lines alone has most of the occupation fraction in the weak field regime but most of the magnetic flux is in form of strong fields. In the case of the magnetic energy this effect is more pronounced and the strong fields carry almost all the magnetic energy of the quiet Sun as it was expected by Socas-Navarro \& Sánchez Almeida (2003; see also Sánchez Almeida 2004)

\subsubsection{Simultaneous inversions}

The simultaneous inversion of the visible and IR lines was successful for $30 \%$ of the classes. The inversion was performed with an initial model with three magnetic components. This initial guess assumed a combination of the models obtained from the inversion of the respective class separately for the IR and visible lines. Figure 4.26 shows some examples. It is noticeable that most of these successful inversions correspond to classes with IR profiles more likely to be produced by weak fields (with no extended tails, e.g., classes 2, 13, 22). The inversion of the IR profiles in Section 4.6.1 infers only weak intrinsic fields for these classes, however the joint inversion of the four lines results in at least one of the components with strong fields. The magnetic fields displayed for each class in Fig. 4.26 are in order of importance (the component with largest mass has a magnetic field $B_{1}$ ). Although the strong fields correspond to minor components for these classes their importance is still high since the flux of these components is comparable or larger than those from the weak field component. The consequence of these results is that there are more strong magnetic fields in the quiet Sun than those inferred only with the IR lines with a MISMA inversion (as was expected by the analysis of the PDFs). The use of the two pairs of lines is mandatory to extract a more reliable information on the distribution of the field strength in the quiet Sun. 
Class 2: $\quad \mathrm{B}_{1}=1779 \mathrm{G}(+) ; \quad \mathrm{B}_{2}=142 \mathrm{G}(+) ; \quad \mathrm{B}_{3}=1653 \mathrm{G}(+)$

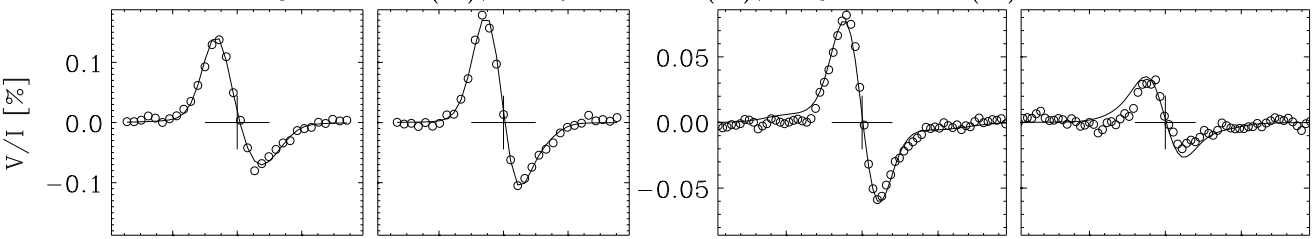

Class 13: $\quad \mathrm{B}_{1}=270 \mathrm{G}(+) ; \quad \mathrm{B}_{2}=1704 \mathrm{G}(+) ; \quad \mathrm{B}_{3}=1700 \mathrm{G}(+)$

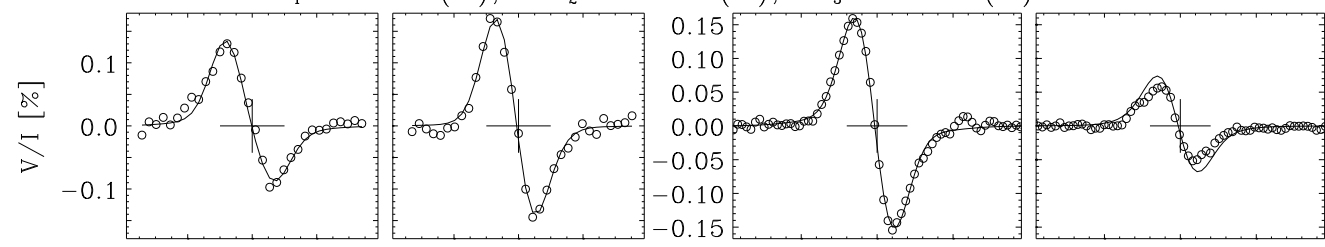

Class 19: $\quad \mathrm{B}_{1}=44 \mathrm{G}(+) ; \quad \mathrm{B}_{2}=247 \mathrm{G}(+) ; \quad \mathrm{B}_{3}=1767 \mathrm{G}(+)$

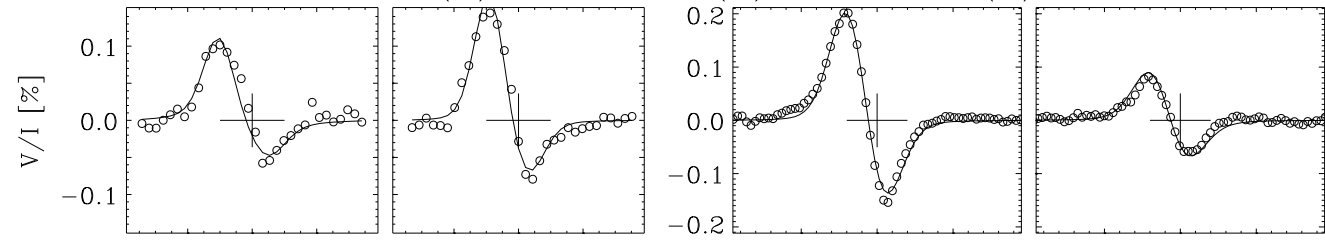

Class 22: $\quad \mathrm{B}_{1}=322 \mathrm{G}(+) ; \quad \mathrm{B}_{2}=1403 \mathrm{G}(+) ; \quad \mathrm{B}_{3}=24 \mathrm{G}(+)$

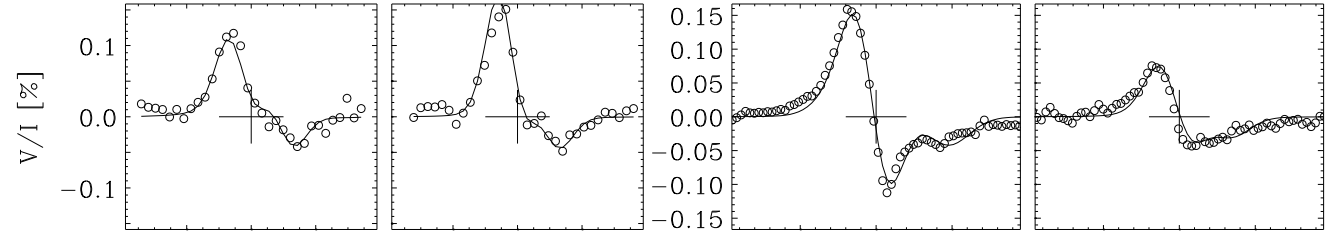

Class 30: $\quad \mathrm{B}_{1}=111 \mathrm{G}(+) ; \quad \mathrm{B}_{2}=1092 \mathrm{G}(-) ; \quad \mathrm{B}_{3}=1046 \mathrm{G}(+)$

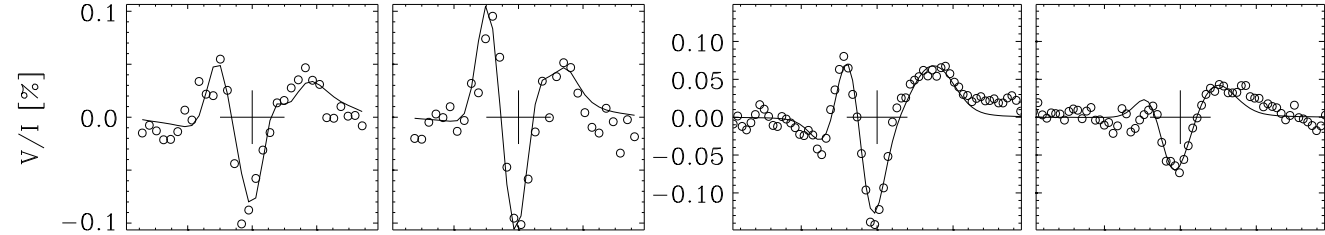

Class 36: $\quad \mathrm{B}_{1}=141 \mathrm{G}(-) ; \quad \mathrm{B}_{2}=598 \mathrm{G}(+) ; \mathrm{B}_{3}=1492 \mathrm{G}(-)$
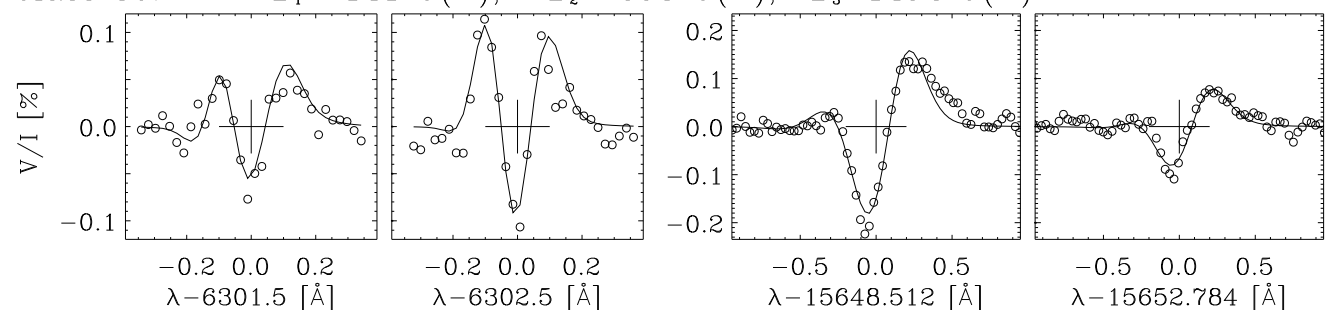

Figure 4.26: Examples of successful simultaneous inversions. The circles represent the observed profiles and the solid lines the fits. The polarity of the magnetic fields is indicated by '+' and '-' signs. 

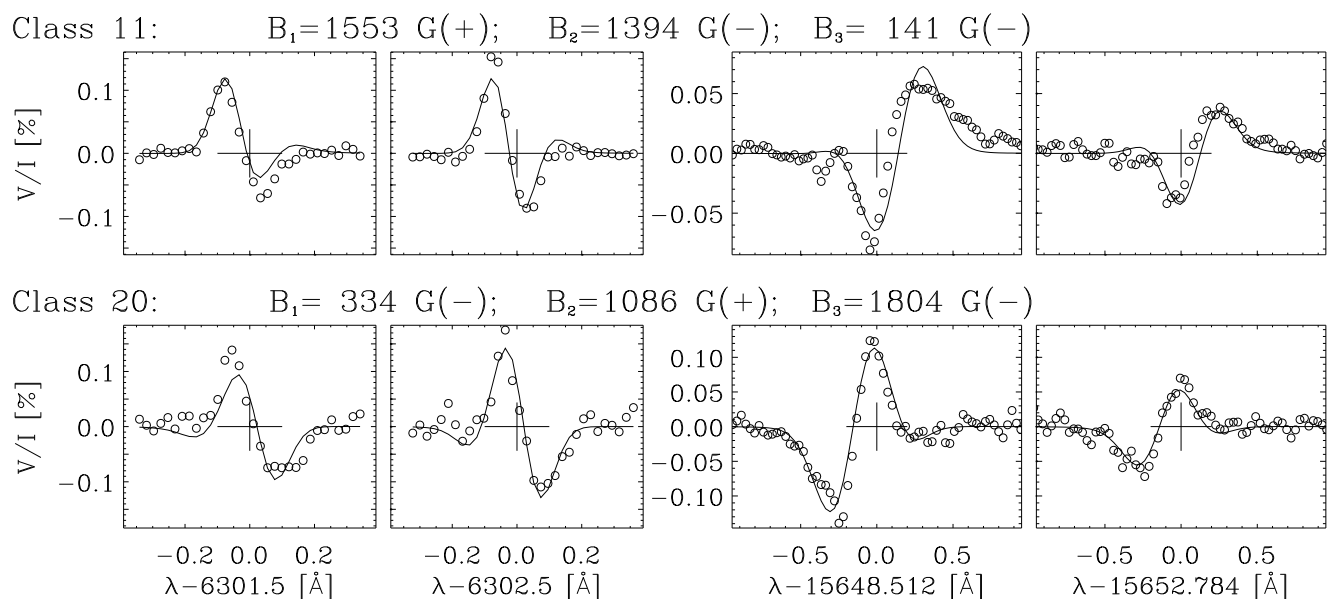

Figure 4.27: Two examples of fits to Stokes $V$ profiles with opposite polarity in the visible and IR pairs of lines produced by the MISMA inversion. The circles represent the observed profiles and the solid lines the fits. The polarity of the magnetic fields is indicated by ' + ' and '-' signs.

Since the MISMA inversion performed was only able to fit some of the classes there are some ingredients, like a mixture of optically thin and thick magnetic flux tubes and dynamics, that should be added in the future to improve the inversion and to retrieve a more complete PDF. Along these lines, one may suspect that it is impossible to fit those profiles with opposite polarity in the visible and IR lines. Figure 4.27 displays the fit to profiles of two classes with this peculiarity. Even though the fit is not satisfactory, the MISMA model was able to produce profiles with opposite polarities very close to the observations, contradictory to expectation. This is possible if one takes into account the behaviour of the Stokes $V$ profiles in visible and IR. For the visible lines the contribution to the polarization is dominated by the strong fields while for the IR lines the weak fields are the predominant contribution. A certain combination of a component with strong fields in one direction and an additional magnetic component with weak fields and opposite polarity will produced very easily profiles like in Fig. 4.27. 


\section{Chapter 5}

\section{Summary and conclusions}

Two innovative observational techniques were performed, in order to study the quiet Sun magnetism. This Chapter summarizes the important results obtained in this Thesis and discusses their consequences.

Magnetograms with a resolution of 0".5 were obtained with the Göttingen FabryPerot Interferometer and with the help of speckle reconstruction techniques (Chapter 3). The sensitivity of the observations allows the detection of magnetic structures in $45 \%$ of the FOV. These magnetic features possess some conspicuous properties:

- The magnetic patches tend to appear in the intergranular lanes were $65 \%$ of the total flux is concentrated. The highest magnetic concentrations trace the mesogranulation providing a new technique for the detection of this convective scale.

- The unsigned magnetic flux density was measured to be $17-21 \mathrm{G}$. This value exceeds, at least by a factor of two, all previous results (Sánchez Almeida et al. 2003; Table 3.1), yielding a new lower limit of the detected flux. The combination of high spatial resolution (a factor of two better than any previous observation of quiet Sun magnetic fields) and the fairly good polarimetric sensitivity are the reasons for detecting more polarization signals than previous studies. Mixed magnetic polarities which were required by other authors to interpret observations of lower resolution (Sánchez Almeida \& Lites 2000; Lites 2002) have been spatially resolved down to scales of 0.5.5. However, there is evidence for a more complex magnetic structuring at still smaller scales. In consequence, when improving further the resolution, more unsigned magnetic flux should be detected. Remembering that the quiet Sun occupies $90 \%$ of the solar surface, the detected flux yields to the conclusion that the intranetwork regions, i.e. the regions in the interior of the quiet Sun network, harbour two times more flux than the active regions 
during maximum of the solar cycle.

- The ratio method applied to the flux measured from the two iron lines $\lambda 6301.5 \AA$ and $\lambda 6302.5 \AA$ yields to the presence of intrinsic fields in the $\mathrm{kG}$ regime. This result does not exclude the existence of sub-kG fields but is the consequence of using the above two Fe I lines which are already known for this tendency to detect strong fields (e.g., Grossman-Doerth et al. 1996; Sánchez Almeida \& Lites 2000; Chapter 4). These kG fields occupy only $2 \%$ of the total area of the observed region.

Chapter 4 was dedicated to the examination of the polarimetric observations performed simultaneously with two pairs of lines in the visible and in the IR spectral ranges, specifically with the Fe I $\lambda 6301.5 \AA$ and Fe I $\lambda 6302.5 \AA$ pair and the Fe I $\lambda 15648 \AA$ and Fe I $\lambda 15652 \AA$ pair. The techniques used to study this unique set of data include different degrees of complexity providing many noticeable results:

- A first analysis provides some evidences that visible and IR lines trace different structures. These are: the magnetic fluxes computed with the magnetograph equation from visible lines is two times larger than the flux computed from IR lines; the presence of flux densities of opposite polarities in the same pixel; the existence of an important imbalanced flux detected with visible lines but negligible when the IR lines are used.

- A further study with inversion techniques under the Milne Eddington approximation reveals the coexistence of $\mathrm{kG}$ and sub-kG field strengths in the pixels analyzed ( $40 \%$ of the FOV). The visible lines detect the $\mathrm{kG}$ fields whereas the IR lines trace the sub-kG fields. Such a phenomenon is produced by the use of a technique assuming a single component model, applied separately to both pairs of lines, in support of the conjecture by Sánchez Almeida \& Lites (2000) and Socas-Navarro \& Sánchez Almeida (2002; 2003). Two new facts are in favour of the independence of the magnetic structures traced by IR and visible lines: 1) the intrinsic field inferred from IR lines does not increase when the field strength obtained from visible lines increase. 2) There is almost no correlation between the velocities derived from both pairs of lines. It is remarkable that the dispersion of velocities obtained in the IR is two times larger than those obtained from visible lines. Assuming the independence of the measurements a probability density function (PDF) for the field strength was computed joining the results from the ME inversion of both pairs of lines.

- Finally, a more reliable analysis was done by using an inversion code based on the MISMA hypothesis (Sánchez Almeida et al. 1996; 
Sánchez Almeida 1997). The inversion was implemented separately for the visible and IR lines. An atmosphere with two magnetic components was assumed for the inversions. The results inferred from the visible lines are very similar to those obtained by Sánchez Almeida \& Lites (2000; see also Socas-Navarro \& Sánchez Almeida 2002) with the difference that in this study some intrinsic weak fields corresponding to the low polarization signals are found. The most conspicuous results come from the inversion of the IR lines (which was the first ever carried out for these lines). It shows the presence of $\mathrm{kG}$ fields in many analyzed pixels $(65 \%$ with $B>500 \mathrm{G}$ ). The contributions to the Stokes $V$ profiles of this strong component were highly spread through the wavelengths due to the strong gradients in height of the magnetic field. Therefore the strong field component was hidden by the weak fields which produce the dominant lobes in the Stokes $V$ profiles. This agrees with the reasonning of Sánchez Almeida \& Lites (2000). Since the inversion of Stokes $V$ profiles yields strong fields only in pixels with sufficiently strong polarization signals, well above noise, it could be that the strong field contributions were often not inferred by the MISMA inversion of the IR lines because they were hidden by noise. However, this could also be a true solar phenomenon and weak fluxes are associated with weaker intrinsic field strengths.

- The PDF computed from ME inversion and that from MISMA inversion of the IR lines have similar properties. The fields in the sub-kG regime occupy most of the magnetized quiet Sun, but the areas with intrinsic fields above $500 \mathrm{G}$ carry most of the magnetic flux (65-80\%) and most of the magnetic energy (90-95\%). Such PDFs are in agreement with those suggested by Socas-Navarro \& Sánchez Almeida (2003) and solve the apparent controversy between visible and IR measurements (Section 2.3)

The high resolution magnetograms show a highly dynamical interaction between the magnetic fields and granulation. There are many observed phenomena suggesting this property: the concentration of magnetic flux in intergranular lanes; the long-lived magnetic structures as trace of mesogranules; the complex distribution of patches with opposite polarity in contact and the short time variation of the distribution. Such behaviour was predicted by magnetohydrodynamical simulations of granulation, whether their magnetic fields are produced by a local dynamo (Cattaneo 1999; Emonet \& Cattaneo 2001), by turbulence from the global dynamo (Stein \& Nordlund 2002), or by re-processing of fields from old active regions (Vögler 2003; Vögler \& Schüssler 2003). Such theories can explain the origin of the sub-kG fields inferred from IR lines. However, the presence of the $\mathrm{kG}$ field strength component which carries most of the unsigned flux needs an additional mechanism to enhance and to concentrate intrinsic fields, e.g. the thermal relaxation (Sánchez Almeida 2001) and the convective collapse (Spruit 1979). 
Two more evidences must be taken into account in the search for explanations of the origin. First, the unsigned flux detected was ten times larger than the mean (signed) flux. Second, this imbalanced flux density was up to $2 \mathrm{G}$ for the $\mathrm{kG}$ component and much lower for the sub-kG fields. This signed flux has the same sign as the network patches surrounding the intranetwork quiet Sun region.

The strong magnetic fields observed at high spatial resolution were not associated with brightness in the intensity images. However, it was evident that spatial resolution was not high enough to resolve the magnetic structures in the intensity images. Recent observations of images taken with a G-band filter with improved spatial resolution show a large number of bright points (Sánchez Almeida et al. 2004) but not sufficient to account for all magnetic signals detected in Chapter 3. Hence, we are still far from resolving the magnetic structures of the intranetwork.

The occupation fraction of the magnetic fields inferred with techniques based on the Zeeman effect is never more than $2 \%$ of the quiet Sun regions analyzed here and in previous works. The rest of the quiet Sun may be occupied by a tangled field which has been recently measured via a novel Hanle effect diagnostics technique (see Shchukina \& Trujillo Bueno 2003; Trujillo Bueno et al. 2004) ${ }^{1}$. These authors concluded that there exists a volume-filling tangled magnetic field on subresolution scales whose average strength is $\sim 130 \mathrm{G}$, which is much stronger in the intergranular regions of solar surface convection than the granular. This Hanleeffect technique is able to conclude on the existence of hidden, mixed-polarity fields on subresolution scales by comparing the observed scattering polarization in spectral lines with that expected for the zero-field reference case (see the review by Trujillo Bueno 2001). The use of this promising technique is important to put together the puzzle of the quiet Sun magnetism.

Finally, the main result of this Thesis is underlined: It has been demonstrated that the use of only the visible or the IR lines in the study of the intrinsic fields creates a bias in the results. However, notice how an appropriate inversion of the IR lines shows both strong and weak field strengths co-existing. In any case, the simultaneous inversion of the lines in both spectral ranges turns out to be mandatory if one wants to obtain a reliable PDF.

The solar physics community is starting to understand the importance that the quiet Sun magnetic fields may have in the solar magnetism, but we are still in development. This Thesis has shown some of the properties and has solved some questions but pointed out some new issues. Until now, only few quiet Sun regions have been analyzed and we cannot ensure the homogeneity of the magnetism in the quiet Sun due to its possible relation with the surrounding active regions. What is the origin of these magnetic fields and what is the relevance of the quiet Sun magnetism for the rest of the Sun? These are questions for the near future.

\footnotetext{
${ }^{1}$ The scattering polarization is produced by the anisotropic illumination of the atoms in the solar atmosphere and is usually observed close to the limb.
} 


\section{References}

[1] Asplund, M., Nordlund, Å., Trampedach, R., Allende Prieto, C., \& Stein, R. F. 2000, Line formation in solar granulation. I. Fe line shapes, shifts and asymmetries, A\&A 359, 729

[2] Ballesteros, E., Collados, M., Bonet, J. A., Lorenzo, F., Viera, T., Reyes, M., \& Rodríguez Hidalgo, I. 1996, Two-dimensional, high spatial resolution, solar spectroscopy using a correlation tracker. I. Correlation tracker description, A\&AS 115, 353

[3] Bellot Rubio, L. R., \& Collados, M. 2003, Understanding internetwork magnetic fields as determined from visible and infrared spectral lines, A\&A 406, 357

[4] Bellot Rubio, L. R., Rodríguez Hidalgo, I, Collados, M., Khomenko, E., \& Ruiz Cobo, B. 2001, Observation of Convective Collapse and Upwardmoving Shocks in the Quiet Sun, ApJ 549, 139

[5] Bellot Rubio, L. R., Ruiz Cobo, B., \& Collados, M. 1997, Flux-Tube Model Atmospheres and Stokes V Zero-crossing Wavelengths, ApJ 478, 45

[6] Bendlin, C. 1993, Hochauflösende zweidimensionale Spektroskopie der solaren Granulation mit einem Fabry-Perot-Interferometer, Ph.D. Thesis, Göttingen University

[7] Bendlin, C. \& Volkmer, R. 1995, The two-dimensional spectrometer in the German Vacuum Tower Telescope/Tenerife. From observations to results, A\&AS 112, 371

[8] Bendlin, C., Volkmer, R., \& Kneer, F. 1992, A new instrument for high resolution, two-dimensional solar spectroscopy, A\&A 257, 817

[9] Bianda, M., Stenflo, J. O., \& Solanki, S. K. 1998, Hanle diagnostics of solar magnetic fields: the SR II 4078 Angstrom line, A\&A 337, 449

[10] Bianda, M., Stenflo, J. O., \& Solanki, S. K. 1999, Hanle effect observations with the CA BT I 4227 line, A\&A 337565 
[11] Bommier, V., \& Rayrole, J. 2002, Search for polarimetric sensitivity in the first observations with THEMIS spectropolarimetric mode MTR (August 1998 campaign), A\&A 381, 227

[12] Borrero, J. M., \& Bellot Rubio, L. R. 2002, A two-component model of the solar photosphere from the inversion of spectral lines, A\&A 385, 1056

[13] Borrero, J. M., Bellot Rubio, L. R., Barklem, P. S., \& del Toro Iniesta, J. C. 2003, Accurate atomic parameters for near-infrared spectral lines, A\&A 404, 749

[14] Bracewell, R. N., 1986, The Fourier Transform and Its applications, $2^{\text {nd }}$ Ed. McGraw-Hill Book Company.

[15] Brault, J. W., \& Neckel, H. 1987, Spectral Atlas of Solar Absolute Diskaveraged and Disk-center Intensities from 3900 to $12510 \AA$, unpublished

[16] Cattaneo, F. 1999, On the Origin of Magnetic Fields in the Quiet Photosphere, ApJ 515, L39

[17] Cattaneo, F., Lenz, D., \& Weiss, N. 2001, On the Origin of the Solar Mesogranulation, ApJ, 563, L91

[18] Chandrasekhar, S. 1950, Radiative Transfer, Oxford University Press.

[19] Collados, M. 1999, High Resolution Spectropolarimetry and Magnetography, in B. Schmieder, A. Hofmann, J. Staude (eds.), Solar magnetic fields and oscillations, Vol. 184, ASP Conf. Series, p. 3

[20] Collados, M. 2003, Stokes polarimeters in the near-infrared, Proc. SPIE, 4843,55

[21] de Boer, C. R. 1993, Speckle-Interferometrie und ihre Anwendungen auf die Sonnenbeobachtung, Ph.D. Thesis, Göttingen University.

[22] de Boer, C. R. 1996, Noise filtering in solar speckle masking reconstructions, A\&AS 120, 589

[23] de Boer, C. R. Kneer, F., \& Nesis, A. 1992, Speckle observations of solar granulation, A\&A 257, L4

[24] Deubner, F. L. 1989, Mesogranulation - A convective phenomenon, A\&A 216, 259

[25] Domínguez Cerdeña, I. 2003, Evidence of mesogranulation from magnetograms of the Sun, A\&A, 412, L65 
REFERENCES

[26] Domínguez Cerdeña, I., Kneer, F., \& Sánchez Almeida, J. 2003a, Quiet-Sun Magnetic Fields at High Spatial Resolution, ApJ, 582, L55

[27] Domínguez Cerdeña, I., Sánchez Almeida, \& Kneer, F. 2003b, Inter-network magnetic fields observed with sub-arcsec resolution, A\&A, 407, 741

[28] Emonet, T., \& Cattaneo, F. 2001, Small-Scale Photospheric Fields: Observational Evidence and Numerical Simulations, ApJ 560, 197

[29] Everitt, B. S. 1995, Cluster Analysis, (Londond: Arnold)

[30] Faurobert-Scholl, M., Feautrier, N., Machefert, F., Petrovay, K., \& Spielfiedel, A 1995, Turbulent magnetic fields in the solar photosphere: diagnostics and interpretation, A\&A, 298, 289

[31] Frutiger, C. \& Solanki, S. K. 1998, Do solar magnetic elements harbor downflows?, A\&A 336, 65

[32] Grossmann-Doerth, U., Keller, C. U., \& Schüssler, M. 1996, Observations of the quiet Sun's magnetic field, A\&A, 315, 610

[33] Gurtovenko, E. A., \& Kostic, R. I. 1981, On the Establishment of Internally Consistent Solar Scales of Oscillator Strengths and Abundances of Chemical Elements - Part Two - on the Errors of the Oscillator Strengths of FEI Lines in the Kurucz, A\&A 101, 132

[34] Harvey-Angle, K. L. 1993, Ph.D. Thesis, Utrecht University, Utrecht

[35] Higgs, L. A. 1962, Asymmetry of solar line profiles, MNRAS 124, 51

[36] Hirzberger, J., Vázquez, M., Bonet, J. A., Hanslmeier, A., \& Sobotka, M. 1997, Time Series of Solar Granulation Images. I. Differences between Small and Large Granules in Quiet Regions, ApJ 480, 406

[37] Janßen, K. 2003, Struktur und Dynamik kleinskaliger Magnetfelder der Sonnenatmosphäre, Ph.D. Thesis, Göttingen University.

[38] Keller, C. U., \& von der Lühe, O. 1992, Solar speckle polarimetry, A\&A 261, 321

[39] Khomenko, E. V., Collados, M., Solanki, S. K., Lagg, A., \& Trujillo Bueno, J. 2003, Quiet-Sun inter-network magnetic fields observed in the infrared, A\&A, 408, 1115

[40] Knox, K. T., \& Thompson, B. J. 1974, Recovery of images from atmospherically degraded short-exposure photographs, ApJ, 193, L45 
[41] Korff D., 1973, Analysis of a Method for Obtaining Near Diffraction Limited Information in the Presence of Atmospheric Turbulence, OSAJ, 63, 971

[42] Koschinsky, M., Kneer, F., \& Hirzberger, J. 2001, Speckle spectropolarimetry of solar magnetic structures, A\&A 365, 588

[43] Koschinsky, M. 2001, Spektroskopie und Polarimetrie kleinskaliger magnetischer Strukturen der Sonnenoberfläche mit Methoden der Bildrekonstruktion, Ph.D. Thesis, Göttingen University

[44] Krieg, J., Wunnenberg, M., Kneer, F., Koschinsky, M., \& Ritter, C. 1999, Height variation of the solar granulation, A\&A 343, 983

[45] Kurucz, R. L. 1994, Atomic Data for Fe and Ni, SAO, CDROM 22

[46] Labeyrie, A. 1970, Attainment of Diffraction Limited Resolution in Large Telescopes by Fourier Analysing Speckle Patterns in Star Images, A\&A 6, 85

[47] Landi Degl'Innocenti, E. 1992, Magnetic field measurements, in Solar Observations: Techniques and Interpretation, ed. F. Sánchez, M. Collados, \& M. Vázquez (Cambridge: Cambridge University Press), 71

[48] Lin, H., 1995, On the Distribution of the Solar Magnetic Fields, ApJ, 446, 421

[49] Lin, H., \& Rimmele, T. 1999, The Granular Magnetic Fields of the Quiet Sun, ApJ, 514, 448

[50] Lites, B. W. 2002, Characterization of Magnetic Flux in the Quiet Sun, ApJ, 573, 431

[51] Livingston, W. C., \& Harvey, J. 1975, A New Component of Solar Magnetism - The Inner Network Fields, BAAS, 7, 346

[52] López Ariste, A., Raylore, J., \& Semel, M. 2000, First results from THEMIS spectropolarimetric mode, A\&AS 142, 137

[53] Martínez Pillet, V., Collados, M, Sánchez Almeida, J. et al. 1999, LPSP \&6 TIP: Full Stokes Polarimeters for the Canary Islands Observatories, in T. R. Rimmele, K. S. Balasubramanian, R. R. Radick (eds.), High resolution solar physics: theory, observations and techniques, Vol. 183, ASP Conf. Series, 264

[54] Mein, P., \& Rayrole, J. 1985, Themis solar telescope, Vistas in Astronomy 28,567

[55] Moore, C. E., Minnaert, M. G. J., \& Houtgast, J 1966, The solar spectrum 2935 A to 8770 A, (Washington: NBS) 
[56] Nave, G., Johansson, S., Learner, R. C. M., Thorne, A. P., \& Brault, J. W. 1994, A new multiplet table for Fe I, ApJS 94, 221

[57] November, L. J., \& Simon, G. W. 1988, Precise proper-motion measurement of solar granulation, ApJ 333, 427

[58] November, L. J., Toomre, J., Gebbie, K. B., \& Simon, G. W. 1981, The detection of mesogranulation on the sun, ApJ, 245, L123

[59] Okunev, O. 2004, Ph.D Thesis, Göttingen University.

[60] Press, W. H., Flannery, B. P., Teukolsky, S. A., \& Vetterling, W. T. 1992, Numerical Recipes in Fortram, $2^{\text {nd }}$ Ed. Cambridge Univ. Press, p. 684

[61] Rayrole, J., \& Mein, P. 1993, THIEMIS Telescope: Prospects in High Resolution Magnetic Field Observations, in H. Zirin, G. Ai, H. Wang (eds.), The magnetic and velocity fields of solar active regions, Vol. 46, ASP Conf. Series, p. 170

[62] Rieutord , M., Roudier, T., Malherbe, J. M., \& Rincon, F. 2000, On mesogranulation, network formation and supergranulation, A\&A 357, 1063

[63] Sánchez Almeida, J. 1997, Physical Properties of the Solar Magnetic Photosphere under the MISMA Hypothesis. I. Description of the Inversion Procedure, ApJ 491, 993

[64] Sánchez Almeida, J. 1998, The unresolved Structure of the Photospheric Magnetic Fiellds: Truths, Troubles and Tricks, in C. E. Alissandrakis \& B. Schmieder (eds.), Three-Dimensional Structure of Solar Active Regions, Vol. 154, ASP Conf. Series, p. 54

[65] Sánchez Almeida, J. 2000, Elusive Magnetic Structures in the Sun and Solar-Type Stars, ApJ, 544, 1135

[66] Sánchez Almeida, J. 2001, Thermal Relaxation of Very Small Solar Magnetic Structures in Intergranules: A Process That Produces Kilogauss Magnetic Field Strengths, ApJ, 556, 928

[67] Sánchez Almeida, J. 2004, The magnetism of the very quiet Sun, in T. Sakurai \& T. Sekii (eds.), The Solar-B mission and the Forefront of Solar Physics, ASP Conf. Series, in press

[68] Sánchez Almeida, J., Domínguez Cerdeña, I., \& Kneer, F. 2003a, Simultaneous Visible and Infrared Spectropolarimetry of a Solar Internetwork Region, ApJ, 597, L177 
[69] Sánchez Almeida, J., Emonet, T., \& Cattaneo, F. 2003b, Polarization of Photospheric Lines from Turbulent Dynamo Simulations, ApJ, 585, 536

[70] Sánchez Almeida, J., \& Landi Degl'Innocenti, E. 1996, MicroStructured Magnetic Atmospheres, SoPh 164, 203

[71] Sánchez Almeida, J., \& Landi Degl'Innocenti, E., Martínez Pillet, V., \& Lites, B. 1996, Line asymmetries and the microstructure of photospheric magnetic fields, ApJ 466, 537

[72] Sánchez Almeida, J., \& Lites, B. W. 2000, Physical Properties of the Solar Magnetic Photosphere under the MISMA Hypothesis. II. Network and Internetwork Fields at the Disk Center, ApJ, 532, 1215

[73] Sánchez Almeida, J., Márquez, I., Bonet, J. A., Domínguez Cerdeña, I., \& Muller, R. 2004, Unresolved bright points in the seemingly non-magnetic quiet Sun, ApJ, submitted

[74] Schlichenmaier, R., \& Collados, M. 2002, Spectropolarimetry in a sunspot penumbra. Spatial dependence of Stokes asymmetries in Fe I 1564.8 nm, A\&A 381, 668

[75] Schüssler, M.,Shelyag, Berdyugina, S., Vögler, A., \& Solanki, S. K. 2003, Why Solar Magnetic Flux Concentrations Are Bright in Molecular Bands, ApJ 597, L173

[76] Shchuckina, N., \& Trujillo Bueno, J. 2003, Towards a Reliable Diagnostics of 'Turbulent' Magnetic Fields Via the Hanle Effect in the Sr I $\lambda 4607 \AA$ Line, in J. Trujillo Bueno, \& J. Sánchez Almeida (eds.), Solar Polarization Workshop 3, Vol. 307, ASP Conf. Series, p. 336

[77] Shelyag, S., Vögler, A., Schüssler, M., \& Solanki, S. K. 2003, Spectral Diagnostics of Magneto-Convection Simulations, in Stars as Suns: Activity, Evolution and Planets, IAU Symp. 219, p.84

[78] Sigwarth, M., Balasubramaniam, K. S., Knölker, M., \& Schmidt, W. 1999, Dynamics of solar magnetic elements, A\&A, 349, 941

[79] Skumanich, A., \& Lites, B. 1987, Stokes profile analysis and vector magnetic fields. I - Inversion of photospheric lines, ApJ 318, 930

[80] Smithson, R. C. 1975, Observations of Weak Solar Magnetic Fields with the Lockheed Diode Array Magnetograph, BAAS, 7, 346 
[81] Socas-Navarro, H. 2001, Stokes Inversion Techniques: Recent Achievements and Future Horizons, in M. Sigwarth (ed.), Advanced Solar Polarimetry - Theory, Observations, and Instrumentation, Vol. 236, ASP Conf. Series, p. 487

[82] Socas-Navarro, H., \& Sánchez Almeida, J. 2002, Magnetic Properties of Photospheric Regions with Very Low Magnetic Flux, ApJ, 565, 1323

[83] Socas-Navarro, H., \& Sánchez Almeida, J. 2003, Magnetic Fields in the Quiet Sun: Observational Discrepancies and Unresolved Structure, ApJ, 593,581

[84] Solanki, S. K. 1986, Velocities in solar magnetic fluxtubes, A\&A 168, 311

[85] Spruit, H. C. 1976, Pressure equilibrium and energy balance of small photospheric fluxtubes, SoPh 50, 269

[86] Spruit, H. C. 1979, Convective collapse of flux tubes, SoPh 61, 363

[87] Stein, R. F., \& Nordlund, Å. 2002, Solar Surface Magneto-Convection and Dynamo Action, in H. Sawaya-Lacoste (ed.), IAU Colloquium 188, 83

[88] Stenflo, J. O. 1973, Magnetic-Field Structure of the Photospheric Network, SoPh 36, 495

[89] Stenflo, J. O. 1982, The Hanle effect and the diagnostics of turbulent magnetic fields in the solar atmosphere, SoPh 80, 209

[90] Stenflo, J. O., \& Harvey, J. W. 1985, Dependence of the properties of magnetic fluxtubes on area factor or amount of flux, SoPh 95, 99

[91] Stenflo, J. O., Solanki, S. K., \& Harvey, J. W. 1987, Diagnostics of solar magnetic fluxtubes with the infrared line Fe I lambda 15648.54 A, A\&A, 173,167

[92] Stix, M. 2002, The sun: an introduction, 2nd ed. Berlin : Springer

[93] Stolpe, F., \& Kneer, F. 2000, On weak magnetic flux structures of the Sun, A\&A 353, 1094

[94] Trujillo Bueno, J. 2001, Atomic Polarization and the Hanle Effect, in M. Sigwarth (ed.), Advanced Solar Polarimetry - Theory, Observations, and Instrumentation, Vol. 236, ASP Conf. Series, p. 161

[95] Trujillo Bueno, J. 2003, Astrophysical Spectropolarimetry and Magnetic Field Diagnostics, in N. E. Piskunov, W. W. Weis, \& D. F. Gray (eds.), Modeling of Stellar Atmospheres, Vol. 210, ASP Conf. Series 
[96] Trujillo Bueno, J., Shchuckina, N., \& Asensio Ramos, A. 2004, A substantial amount of hidden magnetic energy in the quiet Sun., Nature, in press

[97] Tyson, R. K. 1998, Principles of adaptive optics, Boston, MA: Academic Press.

[98] Unno, W. 1956, Line Formation of a Normal Zeeman Triplet, PASJ, 8, 108

[99] Vögler, A. 2003, Three-dimensional simulations of magneto-convection in the solar photosphere, Ph.D. Thesis, Göttingen University.

[100] Vögler, A., \& Schüssler, M. 2003, Studying magneto-convection by numerical simulation, Astron. Nachr., 324, No. 4, 399

[101] von der Lühe, O. 1984, Estimating Fried's parameter from a time series of an arbitrary resolved object imaged through atmospheric turbulence, J. Opt. Soc. Am. A1, 510

[102] von der Lühe, O. 1993, Speckle imaging of solar small scale structure. I - Methods, A\&A, 268374

[103] Wang, J., Wang, H., Tang, F., Lee, J. W., \& Zirin, H. 1995, Flux distribution of solar intranetwork magnetic fields, SoPh 160, 277

[104] Weigelt, G. P. 1977, Modified astronomical speckle interferometry 'speckle masking', Opt. Comm. 21, 55

[105] Weigelt, G. P., \& Wirnitzer, B. 1983, Image reconstruction by the speckle-masking method, Opt. Lett. 8, 389 


\section{Acknowledgements}

Firstly, I would like to thank Franz Kneer, my supervisor. During these three years in Göttingen he always had time for discussion, attending me without problem whenever I knocked his door asking: "Hast du Zeit?". He has been like a father to me, taking care not only of my instruction as a solar physicist, but of my well-being. Thanks Franz, for the long time spent correcting this manuscript.

Special thanks are to Jorge Sánchez. He has been more than a collaborator during this time. He worked at my side like a co-supervisor with a huge interest in the progress of this Thesis and making thousand of suggestions whenever a problem appeared. Without him, this work would have been impossible. He has taught me a lot of solar physics and has increased my interest in the Sun in an order of magnitude.

Much appreciation to my colleagues at the solar group in the Sternwarte, for all the good moments, the very interesting seminars and the life there in general. I specially thank Katja Janßen for instructing me in the speckle reconstruction techniques and letting me use her code. I acknowledge the Max Planck Institut in Lindau for let me join the IMPRS and participate in the seminars. Thanks to the Spanish-speaking mafia working there, for many good times in front of a beer, particularly Juanma who started at the same time I did. I cannot forget the people at the solar group at the IAC for many interesting discussions: Javier, Jose Antonio, Fernando, Manolo, Marian,...

I would also like to thank to Oleg, Katja and Hirzi for supporting in the observations with the FPI; the team of support astronomers and operators at VTT and THEMIS for helping during the simultaneous observations. Special thanks to M. Collados for his assistance in the data reduction of TIP, to Hector Socas who provides the ME inversion code, to Manfred Schüssler and his idea to look for mesogranulation in polarization, and also to Sergey and Alexander for letting me use their MHD simulations.

Thanks to Nazaret, Carol and Gabriela for some corrections of this Thesis.

I acknowledge the support by Deutsche Forschungemeinschaft (DFG) through the grant 418 SPA-112/14/01. Thanks to the IAC for trust me in this enterprise.

Thanks to my parents Juana and Santiago, who sacrificed a lot for the sake of my edducation, to my sister Carolina and my brother David. They all gave me support and love when I needed it.

And finally and most important my infinite gratitude is for Nuribel, my guardian angel. She always believed in me and her support and love was specially important to conclude this Thesis during my last months here. She has shown me the possitive side of life. Thanks Nuria! 


\section{Lebenslauf}

\section{PERSÖNLICHE DATEN}

Name

Geburtstag

Geburtsort

Familienstand

Staatsangehörigkeit
Itahiza Francisco Domínguez Cerdeña

21.12.1979

Puerto del Rosario (Fuerteventura, Spanien)

ledig

Spanisch

\section{SCHULBILDUNG}

1985 - $1993 \quad$ Grundschule am Colegio San José de Calasanz (Fuerteventura, Spanien)

$1993-1997$ Weiter führende Schule an der I.B. Santo

Tomás de Aquino (Fuerteventura, Spanien)

STUDIUM

1997 - $2001 \quad$ Physik (Astrophysik) an der Universidad de La Laguna (Teneriffa, Spanien)

\section{PROMOTION}

$\begin{array}{ll}01.10 .2001-30.09 .2004 & \text { Promotion an der Universitäts-Sternwarte } \\ & \text { Göttingen } \\ & \text { DFG-Stipendium } 418 \text { SPA-112/14/01 }\end{array}$

